\title{
METALLICITY AND TEMPERATURE INDICATORS IN M DWARF $K$-BAND SPECTRA: TESTING NEW AND UPDATED CALIBRATIONS WITH OBSERVATIONS OF 133 SOLAR NEIGHBORHOOD M DWARFS
}

\author{
Bárbara Rojas-Ayala ${ }^{1,2,4}$, Kevin R. Covey $^{2,5,6}$, Philip S. Muirhead ${ }^{3}$, and James P. Lloyd ${ }^{2}$ \\ ${ }^{1}$ Department of Astrophysics, American Museum of Natural History, Central Park West at 79th Street, New York, NY 10024, USA; babs@amnh.org \\ ${ }^{2}$ Department of Astronomy, Cornell University, 122 Sciences Drive, Ithaca, NY 14853, USA \\ ${ }^{3}$ Department of Astronomy, California Institute of Technology, 1200 East California Boulevard, MC 249-17, Pasadena, CA 91125, USA \\ Received 2011 September 6; accepted 2012 January 9; published 2012 March 13
}

\begin{abstract}
We present $K$-band spectra for 133 nearby $(d<33$ ps) M dwarfs, including $18 \mathrm{M}$ dwarfs with reliable metallicity estimates (as inferred from an FGK type companion), $11 \mathrm{M}$ dwarf planet hosts, more than $2 / 3$ of the $\mathrm{M}$ dwarfs in the northern $8 \mathrm{pc}$ sample, and several M dwarfs from the LSPM catalog. From these spectra, we measure equivalent widths of the $\mathrm{Ca}$ and $\mathrm{Na}$ lines, and a spectral index quantifying the absorption due to $\mathrm{H}_{2} \mathrm{O}$ opacity (the $\mathrm{H}_{2} \mathrm{O}-\mathrm{K} 2$ index). Using empirical spectral type standards and synthetic models, we calibrate the $\mathrm{H}_{2} \mathrm{O}-\mathrm{K} 2$ index as an indicator of an $\mathrm{M}$ dwarf's spectral type and effective temperature. We also present a revised relationship that estimates the $[\mathrm{Fe} / \mathrm{H}]$ and $[\mathrm{M} / \mathrm{H}]$ metallicities of $\mathrm{M}$ dwarfs from their Na I, Ca I, and $\mathrm{H}_{2} \mathrm{O}-\mathrm{K} 2$ measurements. Comparisons to model atmosphere provide a qualitative validation of our approach, but also reveal an overall offset between the atomic line strengths predicted by models as compared to actual observations. Our metallicity estimates also reproduce expected correlations with Galactic space motions and $\mathrm{H} \alpha$ emission line strengths, and return statistically identical metallicities for $\mathrm{M}$ dwarfs within a common multiple system. Finally, we find systematic residuals between our $\mathrm{H}_{2} \mathrm{O}$-based spectral types and those derived from optical spectral features with previously known sensitivity to stellar metallicity, such as $\mathrm{TiO}$, and identify the $\mathrm{CaH} 1$ index as a promising optical index for diagnosing the metallicities of near-solar M dwarfs.
\end{abstract}

Key words: stars: abundances - stars: fundamental parameters - stars: late-type

Online-only material: color figures, figure set, supplemental data (FITS) file (tar.gz)

\section{INTRODUCTION}

Contrary to their dimness, $\mathrm{M}$ dwarf stars hold significant promise for illuminating the processes that govern the formation and evolution of stars, planets, and the Milky Way. M dwarfs possess masses of $0.6 M_{\odot}>M_{*}>0.08 M_{\odot}$ (Delfosse et al. 2000), straddling the peak of the stellar initial mass function and dominating stellar populations by number (Bastian et al. 2010). With main-sequence lifetimes that exceed a Hubble time, Galactic M dwarfs also represent a complete archeological record of the chemical evolution and star formation history of the Milky Way (e.g., Bochanski et al. 2007). Moreover, M dwarfs are of great interest as potential exoplanet host stars, as all of a planet's observable signals will be significantly easier to detect if it orbits an $\mathrm{M}$ dwarf compared to a similar planet in orbit around a G dwarf (e.g., Nutzman \& Charbonneau 2008).

Calibrating the fundamental parameters of $\mathrm{M}$ dwarfs, however, is a difficult challenge from both an observational and theoretical perspective. Empirical measurements of M dwarf masses, luminosities, temperatures, and radii are anchored primarily by the information extracted from the orbits of $\mathrm{M}$ dwarf binaries, either the very rare eclipsing systems ( $\sim 15$ systems known; e.g., Kraus et al. 2011; Irwin et al. 2011) or less rare (but also somewhat less informative) spectroscopic/astrometric systems (e.g., Shkolnik et al. 2010; Martinache et al. 2007). Overcoming the intrinsic faintness of these low-luminosity systems, however, makes their characterization a taxing observational challenge,

\footnotetext{
4 Z. Carter Patten '25 Fellow.

5 Hubble Fellow.

6 Visiting Researcher, Department of Astronomy, Boston University, 725

Commonwealth Ave, Boston, MA 02215, USA.
}

and analyses of known binaries reveal systematic offsets in inferred temperatures and radii that correlate with both orbital period and tracers of magnetic activity (Ribas 2006; LópezMorales 2007), suggesting that more wide binaries are needed to accurately infer the parameters typical isolated $\mathrm{M}$ dwarfs (Kraus et al. 2011). Theoretical constraints on M dwarf parameters have been similarly difficult to achieve: accurately modeling the deep convective zones in M dwarf interiors (Mullan \& MacDonald 2001; Browning 2008) and the mixture of molecules and grains that dominate M dwarf atmospheres (Tsuji et al. 1996; Allard et al. 2000) requires significant computational resources, as well as extensive databases of oscillator strengths and opacities measured from laboratory experiments. Confronting theoretical predictions with empirical measurements have also identified significant offsets even for isolated field stars: empirical effective temperature scales appear $\sim 200-300 \mathrm{~K}$ cooler than would be inferred from theoretical model atmospheres (e.g., Jones et al. 2005), for example.

While M dwarf masses, temperatures, and radii have proven challenging to calibrate, those parameters appear strikingly pedestrian when contrasted with the difficulty of inferring M dwarf metallicities. Mould (1976a) was the first to systematically assess the strengths of $\mathrm{M}$ dwarf spectral features as a function of metallicity, constructing a grid of synthetic model atmospheres that included molecular opacities and spanned temperatures of $4250-3000 \mathrm{~K}$ and metallicities of $[\mathrm{Fe} / \mathrm{H}]=$ $-2.0-0.0$ dex. With these models, Mould established several foundations of subsequent investigations of $\mathrm{M}$ dwarf metallicities, such as the metallicity sensitivity of $\mathrm{TiO}$ absorption, the gravity sensitivity of $\mathrm{CaH}$ absorption, and the rough metallicities of Galactic M subdwarfs $([\mathrm{Fe} / \mathrm{H}] \sim-1.0$ dex; Mould 1976b). These studies enabled the subsequent photometric and 
spectroscopic identification of M subdwarfs (Stauffer \& Hartmann 1986; Ruiz \& Anguita 1993), the development of increasingly detailed models of M dwarf atmospheres (Allard 1990), and represent the fundamental origin of the $\mathrm{TiO}$ and $\mathrm{CaH}$-based indices used today to identify metal-poor M subdwarfs (Gizis 1997; Woolf \& Wallerstein 2005; Lépine et al. 2007).

While techniques to identify metal-poor $\mathrm{M}$ dwarfs have been developing for more than 35 years, methods for identifying metal-rich $\mathrm{M}$ dwarfs have received significantly less attention. Interest in identifying $\mathrm{M}$ dwarfs with super-solar metallicities was only recently spurred by the realization that such stars could be highly promising targets for searches for Earth-like planets. The existence of a correlation between (gas giant) planet frequency and host-star metallicity is well established for FGK-dwarfs (Gonzalez 1997; Santos et al. 2004; Valenti $\&$ Fischer 2005), but the first investigations to examine if the planet-metallicity correlation extended to $M$ dwarf hosts returned mixed results (Bonfils et al. 2005a; Bean et al. 2006b). Bonfils et al. (2005a, B05 hereafter) analyzed $V$ and $K$ photometry for 20 wide $\mathrm{M}$ dwarf companions to FGK-dwarfs whose metallicities could be determined reliably via standard spectroscopic techniques. Assuming that both binary components inherit the same metallicity from their parent molecular cloud material, B05 assigned the metallicity measurements for each primary to the $\mathrm{M}$ dwarf secondary, and derived isometallicity contours in the $M_{V}$ versus $V-K_{s}$ color-magnitude plane. This photometric metallicity calibration suggested that nearby M dwarfs, including the planet hosts Gl 876 and Gl 436, were slightly metal-poor compared to the mean metallicity of the Galactic disk. This conclusion was reinforced by the work of Bean et al. (2006b), whose spectroscopic analysis indicated subsolar metallicities for Gl 436, Gl 581, and Gl 876.

The persistence of the planet-metallicity correlation into the $\mathrm{M}$ dwarf regime has been suggested, however, by the recent recalibration of the photometric metallicity calibration by Johnson \& Apps (2009). Using six M dwarfs with wide, metal-rich FGK companions to update the $M_{V}$ versus $V-K$ metallicity contours, Johnson \& Apps (2009, JA09 hereafter) found that the B05 calibration systematically underestimated the compositions of these metal-rich stars. JA09 used their revised calibration to estimate the metallicities of six $\mathrm{M}$ dwarfs with planetary mass companions, concluding that $\mathrm{M}$ dwarf planet hosts are indeed preferentially metal rich, just like FGK hosts. This conclusion was supported by the subsequent work of Schlaufman \& Laughlin (2010), who updated the B05 and JA09 calibrations by using theoretical models to inform the functional form of the $M_{K}$ versus $V-K$ relation, and by inferring the mean metallicity of $\mathrm{M}$ dwarfs in the solar neighborhood from a volume-limited and kinematically matched sample of $F \& G$ dwarfs in the Geneva-Copenhagen Survey (Nordström et al. 2004).

The photometric metallicity calibration developed by B05 and extended by JA09 and SL10 has proven to be a valuable resource, but its dependence on precise $V$ magnitudes and trigonometric parallaxes limits its utility to $M$ dwarfs in the immediate solar neighborhood, at least for the remainder of the preGaia era. In a recent contribution, we presented an alternative, spectroscopic technique for estimating the metallicities of nearsolar metallicity M dwarfs (Rojas-Ayala et al. 2010, hereafter RA10). This technique is capable of providing metallicity estimates with an accuracy comparable to that of the photometric technique and requires only a moderate resolution $K$-band spectrum of the $\mathrm{M}$ dwarf target, providing a significantly lower observational burden for empirically estimating the metallicities of distant, near-solar, or super-solar M dwarfs. An example of the utility of this technique is provided by a recent paper on $\mathrm{M}$ dwarf planet hosts identified in the most recent Kepler data release by P. S. Muirhead et al. (2011, submitted).

While RA10 provided a concise introduction to the spectroscopic $K$-band metallicity indicator, space limitations prevented a full exploration of the technique. In this paper, we provide a full analysis of the motivation, calibration, demonstration, and application of the technique using spectra we obtained for 133 solar neighborhood $M$ dwarfs. In Section 2, we describe the composition of our sample, and the acquisition and reduction of our spectroscopic observations. In Section 3, we describe our measurements of the new $\mathrm{H}_{2} \mathrm{O}-\mathrm{K} 2$ (a modified version of the $\mathrm{H}_{2} \mathrm{O}-\mathrm{K}$ index utilized by RA10), $\mathrm{NaI}$, and $\mathrm{Ca}$ I spectral features upon which the $K$-band metallicity technique depends. In Section 4, we analyze model atmospheres to demonstrate the $\mathrm{H}_{2} \mathrm{O}-\mathrm{K} 2$ index's insensitivity to metallicity, and use the $\mathrm{H}_{2} \mathrm{O}-\mathrm{K} 2$ measurements from our sample to calibrate the index as a spectral type and $T_{\text {eff }}$ indicator. In Section 5 , we use the $\mathrm{H}_{2} \mathrm{O}-\mathrm{K} 2$ index as the basis for a revised $K$-band metallicity calibration; by incorporating an additional $\mathrm{M}$ dwarf binary into our sample of metallicity calibrators, and adopting a modified functional form to better normalize the temperature dependence of the $\mathrm{Na}$ and $\mathrm{Ca}$ features, we derive an updated $K$-band $[\mathrm{Fe} / \mathrm{H}]$ metallicity indicator, as well as a new calibration for overall metallicity $([\mathrm{M} / \mathrm{H}])$, along with robust estimates of the uncertainty in each calibration. In Section 6, we perform several sanity checks of the $K$-band metallicity technique, demonstrating that the $K$-band metallicity estimates preserve expected correlations between metallicity and Galactic kinematics as well as signatures of chromospheric activity. We conclude in Section 7 by using the $K$-band metallicity estimates, in combination with other stellar parameters such as rotational velocity $(v \sin i)$, magnetic activity (as diagnosed by $\mathrm{H}_{\alpha}$ emission), and optical brightness, to identify a sample of nearby $\mathrm{M}$ dwarfs with particular promise for exoplanet surveys. We summarize our findings in Section 8 and present our full $K$-band spectral atlas in the Appendix (we also make our spectra available to the community online).

\section{SAMPLE SELECTION AND OBSERVATIONS}

We observed 133 nearby $M$ dwarfs with declinations higher than $-30^{\circ}$. Our sample consists of the following.

1. $18 \mathrm{M}$ dwarfs with wide ( $>5^{\prime \prime}$ separation), common-propermotion solar-type companions to serve as metallicity calibrators. The FGK-primaries have spectroscopic metallicity measurements by Valenti \& Fischer (2005, SPOCS Catalogue), obtained by fitting synthetic atmospheric spectra to their high-resolution, high signal-to-noise echelle spectra. The SPOCS $[\mathrm{Fe} / \mathrm{H}]$ and $[\mathrm{M} / \mathrm{H}]$ values for the FGKprimaries have been assumed to also describe the metallicities of their $\mathrm{M}$ dwarf companions. This assumption is justified if both binary components formed together, from the same well-mixed molecular cloud, and no mass transfer or dredge-up has occurred in the system. This assumption is supported by the measurements of the metallicities of binaries with two FGK components: Desidera et al. (2004, 2006) find typical metallicity differences of $\leqslant 0.02$ dex between components of binary systems. The binary systems were selected from the Gliese \& Jahreiss (1991) catalog of nearby stars, the Poveda et al. (1994) catalog of nearby wide binary 
and multiple systems, and the list of new HIPPARCOS binaries by Gould \& Chanamé (2004). The SPOCS metallicity values are shown in Table 5. The stars in the calibration sample have $[\mathrm{Fe} / \mathrm{H}]$ metallicities from -0.69 dex to +0.31 dex, $[\mathrm{M} / \mathrm{H}]$ metallicities from -0.49 dex to $+0.25 \mathrm{dex}$, and spectral types from M1 to M6.

2. $115 \mathrm{M}$ dwarfs within $33 \mathrm{pc}$, including $11 \mathrm{M}$ dwarf planet hosts observable from Palomar Mountain: Gl 876 (Marcy et al. 1998; Delfosse et al. 1998a), Gl 436 (Butler et al. 2004), Gl 581 (Bonfils et al. 2005b), Gl 849 (Butler et al. 2006), Gl 176 (Forveille et al. 2009), GJ 1214 (Charbonneau et al. 2009), Gl 649 (Johnson et al. 2010), HIP 57050 (aka GJ 1148; Haghighipour et al. 2010), HIP 79431 (Apps et al. 2010), Gl 179 (Howard et al. 2010), and HIP 12961 (Forveille et al. 2011). More than half are members of the northern 8 pc sample; the remainder were selected from the LPSM catalog (Lépine \& Shara 2005).

Near-infrared spectra of these stars were obtained with the TripleSpec spectrograph on the Palomar 200 inch Hale Telescope (Herter et al. 2008) during several observing runs between 2007 and 2010. TripleSpec at Palomar has no moving parts and simultaneously acquires 5 cross-dispersed orders covering $1.0-2.4 \mu \mathrm{m}$ at a resolution of $\lambda / \Delta \lambda \approx 2700$.

The observations were carried out using two different methods.

1. The star was placed at two different positions along the slit, A and B. Four exposures were taken with an ABBA slit-nodding pattern. Sky-subtraction was performed by differencing A and B exposures in each paired nod.

2. The star was placed at five different positions along the slit. This type of observation pattern was preferred since the array showed a significant number of bad pixels. Each of the five exposures were sky-subtracted using a sky-frame made by median combining the five exposures of the star.

These two types of sky-subtraction methods also remove the emission from the instrument and the dark signal from the detector.

Calibration data were taken at the beginning of each night to correct for the non-uniform response between detector pixels. Several exposures of $30 \mathrm{~s}$ were taken with the dome dark (lampoff frames), and while illuminating the instrument with a white light source (lamp-on frames). A master-flatfield was obtained by subtracting the median of the lamp-off frames from the median of the lamp-on frames.

The spectra were reduced using a modified version of the TEDI reduction software (Muirhead et al. 2011). ${ }^{7}$ Each skysubtracted exposure was divided by the normalized masterflatfield, wavelength calibrated and optimally extracted (Horne 1986). The IDL pipeline walks through the stellar spectrum in each order of the calibrated star image, finds the maximum every 10th pixel, and fits a Gaussian to the slit profile. The Gaussian fit therefore contains all of the stellar signal. The spectral orders of TripleSpec are substantially curved, so the pipeline interpolates onto a rectilinear grid with a thin-plate-spline to correct for the slit tilt, using the spatial and wavelength solutions for the detector by Terry Herter. ${ }^{8}$

The one-dimensional M dwarf spectra were telluric corrected using observations of an A0V star with the IDL-based code xtellcor_general by Vacca et al. (2003). The spectrum of an

\footnotetext{
7 http://www.astro.cornell.edu/ muirhead/\#Downloads

8 herter@astro.cornell.edu
}

A0V star is almost free of metal lines: its observed near-infrared spectrum consists mainly of a featureless and smooth continuum superposed by H I absorption from the star's atmosphere, and telluric absorption features contributed by Earth's atmosphere. A telluric spectrum is obtained by removing the hydrogen lines of the A0V star using a high-resolution model of Vega. The target spectrum is then divided by the telluric spectrum constructed from observations of an A0V star obtained near in time and close in airmass to the target.

Finally, the telluric-corrected spectra were flux calibrated using their Two Micron All Sky Survey (2MASS) $K$-band photometry. The flux density of the target star is proportional to the data number count $\left(D_{\lambda}\right)$ after the telluric calibration:

$$
F_{\lambda}=C D_{\lambda}
$$

Then, the star's spectrum can be flux calibrated from its $m_{K_{s}}$ magnitude, the $K_{s}$ spectral response function $R_{K_{s}}$, and the $K_{S^{-}}$band average flux density of an $m_{K_{s}}=0$ star $F_{K_{s}}^{o}$, by finding $C$ :

$$
C=\frac{\int R_{K_{s}} d \lambda}{\int D_{\lambda} R_{K_{s}} d \lambda} F_{K_{s}}^{o} 10^{-0.4 m_{K_{s}}}
$$

The $K_{s}$ magnitudes for all the targets were obtained from the 2MASS All-Sky Catalog of Point Sources ${ }^{9}$ (Skrutskie et al. 2006). The 2 MASS $K_{s}$ spectral response curve and $F_{K_{s}}^{o}$ are available online. $^{10}$

Table 1 presents the properties of each star in the sample, including the star's distance, $V$ and $K_{s}$ magnitudes, the date the spectrum was observed, and the average signal-to-noise ratio $(\mathrm{S} / \mathrm{N})$ obtained in the $K$-band continuum. The full sample of $K$-band spectra are presented in Figure 1; the sample is ordered in Table 1 and in Figure 1 in order of increasing $\mathrm{H}_{2} \mathrm{O}-\mathrm{K} 2$ index (see Section 3.2). Four stars appear to have sub-optimal telluric corrections (Gl 644AB, 829AB, 809, and 908); these stars are flagged in Table 1 and their spectra are presented separately in Figure 1.

\section{SPECTROSCOPIC ANALYSIS}

\subsection{Na and Ca EWs}

RA10 demonstrated that the $2.205 \mu \mathrm{m} \mathrm{Na}$ I and $2.263 \mu \mathrm{m} \mathrm{Ca} \mathrm{I}$ lines can be used to estimate the metallicities of $\mathrm{M}$ dwarf stars. We measured the equivalent widths (EWs) of each of these lines for every star in our sample. The standard definition of the EW of a line is given by the following equation:

$$
\mathrm{EW}_{\lambda}=\int_{\lambda_{1}}^{\lambda_{2}}\left[1-\frac{F(\lambda)}{F_{c}(\lambda)}\right] d \lambda .
$$

Here, $F(\lambda)$ represents the flux across the wavelength range of the line $\left(\lambda_{2}-\lambda_{1}\right)$, and $F_{c}(\lambda)$ represents the estimated continuum flux on either side of the absorption feature.

The EWs of the $\mathrm{Na}$ I and $\mathrm{Ca}$ I features were calculated with an IDL pipeline using the following approximation:

$$
\mathrm{EW}_{\lambda} \simeq \sum_{i=0}^{n}\left[1-\frac{F\left(\lambda_{i}\right)}{F_{c}\left(\lambda_{i}\right)}\right] \Delta \lambda_{i}
$$

\footnotetext{
9 http://vizier.u-strasbg.fr/viz-bin/VizieR?-source=II/246

$10 \mathrm{http}: / /$ www.ipac.caltech.edu/2mass/releases/allsky/doc/sec6_4a.html
} 
Table 1

TripleSpec Nearby M Dwarf Sample—Source Properties

\begin{tabular}{|c|c|c|c|c|c|c|c|}
\hline Name & $\begin{array}{c}d \\
(\mathrm{pc})\end{array}$ & $\begin{array}{c}V \\
(\mathrm{mag})\end{array}$ & $V$ References & 2MASS K & Date & SNR & Star \\
\hline LHS 3576 & 19.9 & 10.29 & 1 & 6.553 & 2009 Jul 20 & 278 & LSPM \\
\hline Gl 338 A & 6.2 & 7.64 & 1 & 3.99 & 2009 Feb 5 & 243 & $8 \mathrm{pc}$ \\
\hline Gl 338 B & 6.3 & 7.7 & 1 & 4.14 & 2009 Feb 5 & 350 & $8 \mathrm{pc}$ \\
\hline G 210-45 & 23.4 & 11.21 & 1 & 7.251 & 2009 Jul 20 & 290 & LSPM \\
\hline Gl 205 & 5.7 & 7.97 & 1 & 4.039 & 2009 Sep 30 & 652 & $8 \mathrm{pc}$ \\
\hline Gl $725 \mathrm{~A}$ & 3.6 & 8.94 & 1 & 4.432 & 2009 Jul 19 & 866 & $8 \mathrm{pc}$ \\
\hline Gl $412 \mathrm{~A}$ & 4.8 & 8.82 & 1 & 4.769 & 2009 Feb 5 & 337 & $8 \mathrm{pc}$ \\
\hline Gl 686 & 8.1 & 9.62 & 1 & 5.572 & 2009 Jul 19 & 702 & LSPM \\
\hline Gl $752 \mathrm{AB}$ & 5.9 & 9.12 & 1 & 4.673 & 2009 Jul 19 & 416 & $8 \mathrm{pc}$ \\
\hline Gl 649 & 10.3 & 9.7 & 1 & 5.624 & 2010 May 22 & 862 & Planet/LSPM \\
\hline HIP 12961 & 23.8 & 10.25 & 1 & 6.736 & 2010 Nov 25 & 268 & Planet/LSPM \\
\hline Gl 212 & 12.5 & 9.78 & 1 & 5.759 & 2009 Sep 30 & 627 & Calibrator/LSPM \\
\hline HD 46375 B & 26.4 & 11.8 & 3 & 7.843 & 2009 Feb 4 & 795 & Calibrator/LSPM \\
\hline HIP 79431 & 14.9 & 11.34 & 1 & 6.589 & 2010 May 22 & 659 & Planet/LSPM \\
\hline$V^{*}$ V1513 Cyg & $\ldots$ & 11.98 & 5 & 8.113 & 2009 Jul 20 & 864 & LSPM \\
\hline Gl 411 & 2.5 & 7.49 & 1 & 3.254 & 2009 Feb 5 & 444 & $8 \mathrm{pc}$ \\
\hline G1 526 & 5.4 & 8.46 & 1 & 4.415 & 2010 May 22 & 1996 & $8 \mathrm{pc}$ \\
\hline V* V547 Cas & 10.1 & 10.27 & 1 & 6.037 & 2007 Nov 17 & 385 & LSPM \\
\hline G1 872 B & 16.3 & 11.7 & 3 & 7.3 & 2009 Jul 20 & 356 & Calibrator/LSPM \\
\hline G1 797 B & 20.1 & 11.88 & 8 & 7.416 & 2009 Jul 19 & 581 & Calibrator/LSPM \\
\hline LHS 3577 & 12.5 & 10.79 & 1 & 6.533 & $2009 \mathrm{Jul} 20$ & 311 & LSPM \\
\hline Gl 581 & 6.3 & 10.57 & 1 & 5.837 & 2009 Feb 5 & 359 & Planet/8 pc \\
\hline Gl 408 & 6.6 & 10.03 & 1 & 5.503 & 2009 Dec 24 & 2079 & $8 \mathrm{pc}$ \\
\hline G1 251 & 5.5 & 9.89 & 1 & 5.275 & 2009 Feb 4 & 422 & $8 \mathrm{pc}$ \\
\hline Gl 297.2 B & 12.5 & 11.8 & 3 & 7.418 & 2008 Feb 16 & 260 & LSPM \\
\hline Gl $250 \mathrm{~B}$ & 8.7 & 10.05 & 3 & 5.723 & 2009 Feb 4 & 681 & Calibrator/LSPM \\
\hline Gl 176 & 9.4 & 9.95 & 1 & 5.607 & 2009 Sep 30 & 667 & Planet/LSPM \\
\hline NLTT 14186 & 34.5 & 14.60 & 8 & 7.621 & 2009 Sep 30 & 630 & Calibrator/LSPM \\
\hline Gl 849 & 8.8 & 10.41 & 1 & 5.594 & 2009 Jul 20 & 755 & Planet/LSPM \\
\hline G1 725 B & 3.5 & 9.7 & 1 & 5 & 2009 Jul 19 & 832 & $8 \mathrm{pc}$ \\
\hline Gl $661 \mathrm{AB}$ & 6.1 & 9.44 & 3 & 4.83 & 2009 Jul 19 & 708 & $8 \mathrm{pc}$ \\
\hline G 262-29 & 32.8 & 11.7 & 2 & 7.61 & 2009 Jul 20 & 425 & LSPM \\
\hline LHS 3605 & 13.7 & 11.98 & 1 & 7.64 & $2009 \mathrm{Jul} 20$ & 307 & LSPM \\
\hline LHS 115 & 10.2 & 12.19 & 3 & 6.377 & 2007 Nov 17 & 326 & LSPM \\
\hline Gl 625 & 6.6 & 10.13 & 1 & 5.833 & 2009 Jul 19 & 573 & $8 \mathrm{pc}$ \\
\hline Gl 643 & 6.5 & 11.73 & 1 & 5.756 & 2009 Jul 19 & 530 & $8 \mathrm{pc}$ \\
\hline Gl 273 & 3.8 & 9.84 & 1 & 4.857 & 2009 Feb 4 & 327 & $8 \mathrm{pc}$ \\
\hline LHS 3591 & 32.4 & 12.73 & 2 & 8.238 & $2009 \mathrm{Jul} 20$ & 288 & LSPM \\
\hline Gl $860 \mathrm{AB}$ & 4 & 9.59 & 1 & 4.777 & 2009 Jul 20 & 304 & $8 \mathrm{pc}$ \\
\hline Gl 687 & 4.5 & 9.15 & 1 & 4.548 & 2010 May 24 & 984 & $8 \mathrm{pc}$ \\
\hline Gl 628 & 4.3 & 10.1 & 1 & 5.075 & 2010 May 24 & 808 & $8 \mathrm{pc}$ \\
\hline Gl 873 & 5 & 10.29 & 1 & 5.299 & 2009 Jul 20 & 392 & $8 \mathrm{pc}$ \\
\hline LHS 3558 & 8 & 10.54 & 1 & 5.933 & $2009 \mathrm{Jul} 20$ & 628 & $8 \mathrm{pc}$ \\
\hline G $168-24$ & 16.3 & 12.51 & 2 & 7.873 & $2009 \mathrm{Jul} 20$ & 752 & LSPM \\
\hline HD 222582 B & 41.9 & 14.50 & 7 & 9.583 & 2009 Jul 20 & 376 & Calibrator/8 pc \\
\hline Gl 436 & 10.2 & 10.67 & 1 & 6.073 & 2008 Feb 17 & 997 & Planet/LSPM \\
\hline HIP 57050 & 11 & 11.86 & 1 & 6.822 & 2010 May 22 & 537 & Planet/LSPM \\
\hline LP 816-60 & 5.5 & 11.41 & 1 & 6.199 & 2009 Jul 19 & 899 & $8 \mathrm{pc}$ \\
\hline Gl 896 A & 6.2 & 10.05 & 1 & 5.326 & $2009 \mathrm{Jul} 20$ & 923 & $8 \mathrm{pc}$ \\
\hline Gl 876 & 4.7 & 10.16 & 1 & 5.01 & $2009 \mathrm{Jul} 20$ & 577 & Planet/8 pc \\
\hline Gl 402 & 5.6 & 11.64 & 1 & 6.371 & 2009 Dec 24 & 1452 & $8 \mathrm{pc}$ \\
\hline Gl 53,1 B & 20.7 & 13.6 & 3 & 8.673 & 2007 Nov 16 & 508 & LSPM \\
\hline Gl 555 & 6.1 & 11.32 & 1 & 5.939 & 2010 May 22 & 716 & $8 \mathrm{pc}$ \\
\hline Gl 179 & 12.1 & 11.94 & 1 & 6.942 & 2010 Nov 25 & 278 & Planet/LSPM \\
\hline LHS 494 & 15.9 & 12.51 & 2 & 7.397 & $2009 \mathrm{Jul} 20$ & 491 & LSPM \\
\hline Gl 388 & 4.9 & 9.4 & 2 & 4.593 & 2009 Feb 5 & 260 & $8 \mathrm{pc}$ \\
\hline Gl 169.1 A & 5.5 & 11.06 & 2 & 5.717 & 2009 Sep 30 & 1508 & $8 \mathrm{pc}$ \\
\hline LHS 3409 & 20.3 & 15.11 & 2 & 10.229 & 2010 May 24 & 280 & LSPM \\
\hline Gl 699 & 1.8 & 9.54 & 1 & 4.524 & 2010 May 24 & 758 & $8 \mathrm{pc}$ \\
\hline LHS 220 & 13.3 & 13.77 & 2 & 8.773 & 2007 Nov 17 & 302 & LSPM \\
\hline Gl 783.2 B & 20.4 & 13.94 & 3 & 8.883 & 2009 Jul 19 & 602 & Calibrator/LSPM \\
\hline Gl 445 & 5.4 & 10.8 & 1 & 5.954 & 2009 Feb 5 & 201 & $8 \mathrm{pc}$ \\
\hline G1 213 & 5.8 & 11.56 & 1 & 6.389 & 2009 Feb 4 & 318 & $8 \mathrm{pc}$ \\
\hline Gl 544 B & 19 & 14.5 & 3 & 9.592 & 2008 Feb 16 & 811 & Calibrator/LSPM \\
\hline
\end{tabular}


Table 1

(Continued)

\begin{tabular}{|c|c|c|c|c|c|c|c|}
\hline Name & $\begin{array}{c}d \\
(\mathrm{pc})\end{array}$ & $\begin{array}{c}V \\
(\mathrm{mag})\end{array}$ & $V$ References & 2MASS K & Date & SNR & Star \\
\hline LHS 1723 & 7.5 & 12.16 & 3 & 6.736 & 2009 Sep 30 & 582 & $8 \mathrm{pc}$ \\
\hline GJ 1224 & 7.5 & 13.64 & 2 & 7.827 & 2009 Jul 19 & 876 & $8 \mathrm{pc}$ \\
\hline GJ 1119 & 10.3 & 13.32 & 2 & 7.741 & 2007 Nov 16 & 972 & LSPM \\
\hline G 041-014 & 5.6 & 10.89 & 3 & 5.688 & 2009 Dec 24 & 857 & $8 \mathrm{pc}$ \\
\hline GJ 3348 B & 23.5 & 13.98 & 3 & 8.791 & 2009 Sep 30 & 416 & Calibrator/LSPM \\
\hline LHS 1809 & 9.3 & 14.45 & 2 & 8.435 & 2007 Nov 17 & 746 & LSPM \\
\hline Gl 447 & 3.3 & 11.12 & 1 & 5.654 & 2009 Feb 5 & 304 & $8 \mathrm{pc}$ \\
\hline LHS 1066 & 16.6 & 14 & 3 & 9.11 & 2007 Nov 17 & 668 & LSPM \\
\hline GJ 3134 & 24 & 14.31 & 3 & 8.995 & 2007 Nov 17 & 499 & LSPM \\
\hline Gl 231.1 B & 19.9 & 13.27 & 3 & 8.267 & 2009 Feb 4 & 908 & Calibrator/LSPM \\
\hline LHS 3593 & 13.9 & 13.98 & 2 & 8.481 & 2009 Jul 20 & 228 & LSPM \\
\hline GJ 3379 & 5.4 & 11.33 & 2 & 6.042 & 2009 Feb 4 & 662 & $8 \mathrm{pc}$ \\
\hline Gl $268 \mathrm{AB}$ & 6.4 & 11.65 & 1 & 5.846 & $2009 \mathrm{Feb} 4$ & 443 & $8 \mathrm{pc}$ \\
\hline G1 768.1 B & 19.4 & 13.1 & 3 & 8.012 & 2010 May 22 & 531 & Calibrator/LSPM \\
\hline NLTT 25869 & 11 & 14.5 & 3 & 8.64 & $2008 \mathrm{Feb} 16$ & 551 & LSPM \\
\hline LHS 6007 & 21.3 & 14.25 & 2 & 8.852 & 2007 Nov 17 & 437 & LSPM \\
\hline Gl 905 & 3.2 & 12.27 & 2 & 5.929 & 2009 Jul 20 & 824 & $8 \mathrm{pc}$ \\
\hline LHS 495 & 9.8 & 13.41 & 2 & 7.749 & 2009 Jul 20 & 258 & LSPM \\
\hline GJ 1214 & 13 & 14.67 & 2 & 8.78 & 2010 May 24 & 415 & Planet/LSPM \\
\hline G 246-33 & 14 & 14.63 & 3 & 8.656 & 2007 Nov 16 & 618 & LSPM \\
\hline Gl 324 B & 12.5 & 13.15 & 3 & 7.666 & 2008 Feb 16 & 814 & Calibrator/LSPM \\
\hline G 203-47 & 7.3 & 11.77 & 1 & 6.485 & 2009 Jul 19 & 659 & $8 \mathrm{pc}$ \\
\hline Gl 299 & 6.8 & 12.83 & 3 & 7.66 & 2009 Feb 4 & 858 & $8 \mathrm{pc}$ \\
\hline LHS 224 & 9.2 & 13.3 & 2 & 7.776 & 2007 Nov 17 & 465 & LSPM \\
\hline Gl $611 \mathrm{~B}$ & 13.8 & 14.2 & 2 & 9.159 & 2008 Feb 16 & 970 & Calibrator/LSPM \\
\hline Gl $630.1 \mathrm{~A}$ & 14.5 & 12.9 & 2 & 7.796 & 2008 Feb 17 & 483 & LSPM \\
\hline NSV 13261 & 15.9 & 14.67 & 2 & 8.753 & $2009 \mathrm{Jul} 20$ & 407 & LSPM \\
\hline NLTT 15867 & 25 & 16.49 & 3 & 10.312 & 2009 Feb 4 & 334 & Calibrator/LSPM \\
\hline Gl $166 \mathrm{C}$ & 5 & 11.17 & 3 & 5.962 & 2009 Sep 30 & 591 & Calibrator/8 pc \\
\hline LHS 3376 & 7.3 & 13.46 & 2 & 7.948 & $2009 \mathrm{Jul} 19$ & 718 & $8 \mathrm{pc}$ \\
\hline GJ 3253 & 14.3 & 13.79 & 2 & 8.053 & 2007 Nov 16 & 417 & LSPM \\
\hline GJ 3069 & 14.4 & 15.12 & 3 & 8.864 & 2007 Nov 17 & 412 & LSPM \\
\hline GJ 1286 & 7.2 & 14.69 & 2 & 8.183 & 2009 Jul 20 & 228 & $8 \mathrm{pc}$ \\
\hline Gl 164 & 11.9 & 13.5 & 2 & 7.915 & 2008 Feb 17 & 495 & LSPM \\
\hline Gl 777 B & 11.7 & 14.33 & 2 & 8.712 & 2009 Jul 19 & 522 & Calibrator/LSPM \\
\hline Gl 866 & 3.4 & 12.18 & 3 & 5.537 & 2009 Jul 20 & 400 & $8 \mathrm{pc}$ \\
\hline Gl $473 \mathrm{AB}$ & 4.3 & 12.44 & 3 & 6.042 & 2009 Feb 5 & 413 & $8 \mathrm{pc}$ \\
\hline Gl $234 \mathrm{AB}$ & 4.1 & 11.12 & 1 & 5.486 & 2009 Feb 4 & 691 & $8 \mathrm{pc}$ \\
\hline LHS 3549 & 9.3 & 14.04 & 2 & 8.095 & 2009 Jul 20 & 442 & LSPM \\
\hline LHS 1706 & 14.1 & 15.23 & 2 & 8.977 & 2009 Sep 30 & 372 & LSPM \\
\hline V* V388 Cas & $\ldots$ & 13.78 & 5 & 7.718 & 2007 Nov 17 & 410 & LSPM \\
\hline LHS 3799 & 7.4 & 13.25 & 2 & 7.319 & 2009 Jul 20 & 388 & $8 \mathrm{pc}$ \\
\hline G1 285 & 5.9 & 11.19 & 1 & 5.698 & 2009 Feb 4 & 1447 & $8 \mathrm{pc}$ \\
\hline LHS 18 & 12 & 14.66 & 2 & 9.083 & 2007 Nov 17 & 475 & LSPM \\
\hline Gl 412 B & 4.8 & 14.45 & 3 & 7.839 & 2009 Feb 5 & 367 & $8 \mathrm{pc}$ \\
\hline LHS 1901 & 8 & 15.87 & 3 & 9.126 & 2007 Nov 17 & 538 & $8 \mathrm{pc}$ \\
\hline LSPM J0011 $^{\mathrm{a}}$ & 11.7 & 15.87 & 3 & 9.093 & 2007 Nov 17 & 666 & LSPM \\
\hline GJ 1245 AC & 4.5 & 13.41 & 2 & 6.854 & 2009 Jul 19 & 539 & $8 \mathrm{pc}$ \\
\hline GJ 1245 B & 4.7 & 13.99 & 3 & 7.387 & 2009 Jul 19 & 440 & $8 \mathrm{pc}$ \\
\hline GJ $1116 \mathrm{AB}$ & 5.2 & 14.06 & 2 & 6.889 & 2009 Feb 5 & 1094 & $8 \mathrm{pc}$ \\
\hline GJ 3146 & 8.5 & 15.79 & 2 & 8.981 & 2007 Nov 17 & 485 & LSPM \\
\hline LHS 252 & 10 & 15.05 & 2 & 8.668 & 2007 Nov 17 & 772 & LSPM \\
\hline Gl 376 B & 13.9 & 16.13 & 3 & 9.275 & $2009 \operatorname{Dec} 24$ & 372 & Calibrator/LSPM \\
\hline Gl 1156 & 6.5 & 13.79 & 2 & 7.57 & 2009 Feb 5 & 505 & $8 \mathrm{pc}$ \\
\hline Gl 406 & 2.4 & 13.54 & 3 & 6.084 & 2009 Dec 24 & 1860 & $8 \mathrm{pc}$ \\
\hline GJ 3147 & 10.4 & 15.99 & 2 & 9.011 & 2007 Nov 16 & 507 & LSPM \\
\hline LHS 292 & 4.5 & 15.6 & 2 & 7.926 & 2009 Dec 24 & 870 & $8 \mathrm{pc}$ \\
\hline Gl $644 \mathrm{C}$ & 6.5 & 16.7 & 3 & 8.816 & 2009 Jul 19 & 542 & $8 \mathrm{pc}$ \\
\hline GJ 1111 & 3.6 & 14.81 & 2 & 7.26 & $2008 \mathrm{Feb} 16$ & 746 & $8 \mathrm{pc}$ \\
\hline LHS 2090 & 6 & 16.1 & 3 & 8.437 & 2009 Dec 24 & 914 & $8 \mathrm{pc}$ \\
\hline V* V492 Lyr & 14.1 & 18.23 & 2 & 10.308 & 2009 Jul 20 & 370 & LSPM \\
\hline Teegardens & 3.6 & 15.4 & 3 & 7.585 & $2009 \mathrm{Feb} 4$ & 1092 & $8 \mathrm{pc}$ \\
\hline 2MASS J18b & 6.2 & 18.27 & 3 & 9.171 & 2009 Jul 19 & 383 & $8 \mathrm{pc}$ \\
\hline LHS 2065 & 8.5 & 18.8 & 2 & 9.942 & $2008 \mathrm{Feb} 16$ & 264 & LSPM \\
\hline
\end{tabular}


Table 1

(Continued)

\begin{tabular}{|c|c|c|c|c|c|c|c|}
\hline Name & $\begin{array}{c}d \\
(\mathrm{pc})\end{array}$ & $\begin{array}{c}V \\
(\mathrm{mag})\end{array}$ & $V$ References & 2MASS K & Date & SNR & $\overline{\text { Star }}$ \\
\hline LHS 2924 & 10.8 & 19.74 & 2 & 10.744 & 2008 Feb 17 & 560 & LSPM \\
\hline Gl $908^{c}$ & 6.0 & 8.98 & 3 & 5.043 & 2009 Jul 20 & 253 & $8 \mathrm{pc}$ \\
\hline Gl $809^{c}$ & 7.0 & 8.54 & 3 & 4.618 & 2009 Jul 19 & 680 & $8 \mathrm{pc}$ \\
\hline Gl $644 \mathrm{AB}^{\mathrm{c}}$ & 6.5 & 9.80 & 3 & 4.403 & 2009 Jul 19 & 587 & $8 \mathrm{pc}$ \\
\hline Gl $829 \mathrm{AB}^{\mathrm{c}}$ & 6.7 & 10.30 & 3 & 5.453 & $2009 \mathrm{Jul} 19$ & 208 & $8 \mathrm{pc}$ \\
\hline
\end{tabular}

Notes.

${ }^{\text {a }}$ LSPM J0011+5908.

b 2MASS J18353790+3259545.

c Stars with low quality $K$-band spectra.

* Reference of distance and $V$ magnitude: (1) HIPPARCOS, van Leeuwen 2007; (2) YALE, van Altena et al. 2001; (3) PMSU, Reid et al. 1997; (4) Koen et al. 2010; (5) Leggett 1992; (6) Bessel 1990; (7) Gould \& Chanamé 2004; (8) Gliese \& Jahreiss 1991.

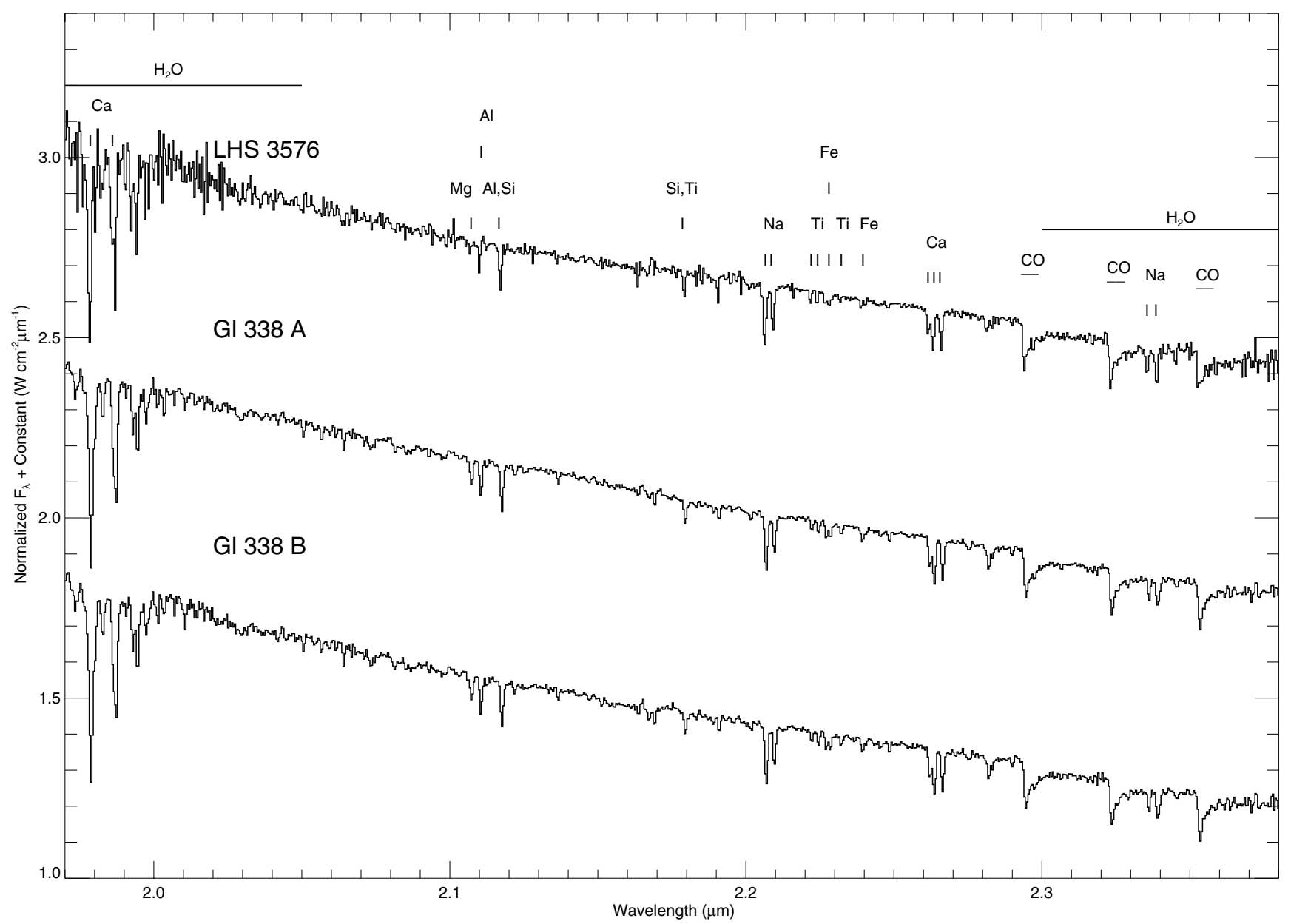

Figure 1. TripleSpec 200 inch $K$-band spectra of 133 nearby M-dwarfs. The main spectral features are indicated.

(The complete figure set (28 images) is available in the online journal.)

Equation (4) is the Riemann sum expression of the integral in Equation (3), where the $F\left(\lambda_{i}\right)$ and $F_{c}\left(\lambda_{i}\right)$ are the line flux and the estimated continuum flux of the wavelength interval $\Delta \lambda_{i}$, respectively, and $n$ is the number of intervals. The integration limits adopted for the $\mathrm{Na}$ I doublet and $\mathrm{Ca}$ I triplet features are shown in Table 2.

Since the continuum of the M dwarf spectra is affected greatly by molecular/broad absorption features, a pseudocontinuum is calculated instead using regions adjacent to the feature of interest and free of any other atomic features. The pseudocontinuum for each feature is estimated from a linear fit to the median flux within $0.003 \mu \mathrm{m}(\sim 7$ pixels) wide regions centered on the continuum points listed in Table 2; the median flux at each continuum point is also weighted by its uncertainty in performing the fit. The continuum points in Table 2 were originally defined by Förster Schreiber (2000) for use in modest resolution spectra of late-type giants.

To estimate the uncertainties on the EW measurements, a Monte Carlo approach was used. An IDL pipeline added random Gaussian noise (based on the signal-to-noise of the spectrum) to the star's spectrum and then calculated the EW of each feature using Equation (4). The entire procedure was repeated 

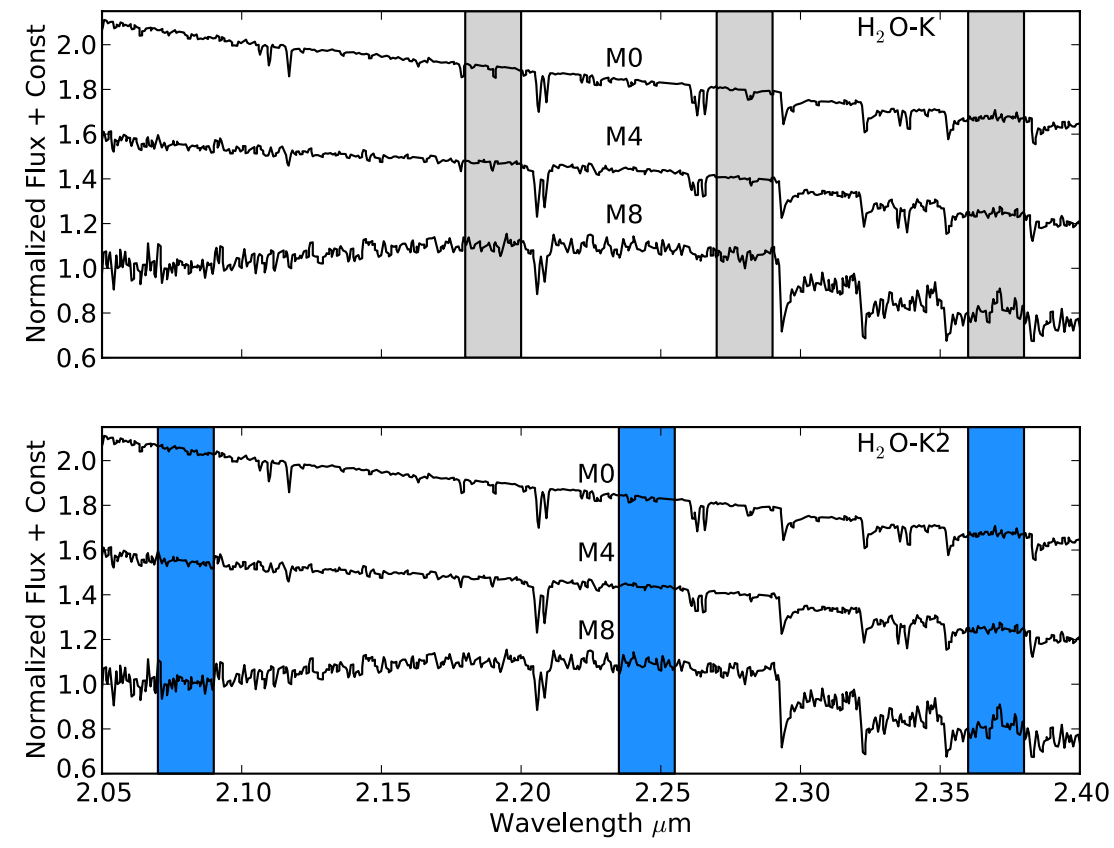

Figure 2. $\mathrm{H}_{2} \mathrm{O}-\mathrm{K}$ index by Covey et al. (2010) vs. the new $\mathrm{H}_{2} \mathrm{O}-\mathrm{K} 2$ index in Equation (5). The portions of the spectrum used by the $\mathrm{H}_{2} \mathrm{O}-\mathrm{K} 2$ index are almost free of atomic features and better sample the change in the overall shape of the spectra of $\mathrm{M}$ dwarfs due to the water absorption from $2.07 \mu \mathrm{m}$ to $2.38 \mu \mathrm{m}$.

(A color version of this figure is available in the online journal.)

Table 2

EW Bandpasses and Continuum Points

\begin{tabular}{lccc}
\hline \hline Feature & $\begin{array}{c}\text { Wavelength } \\
(\mu \mathrm{m})\end{array}$ & $\begin{array}{c}\text { Integration Limits } \\
(\mu \mathrm{m})\end{array}$ & $\begin{array}{c}\text { Continuum Points } \\
(\mu \mathrm{m})\end{array}$ \\
\hline Na I & 2.206 & $2.2020-2.2120$ & $2.1965,2.2125,2.2175$ \\
& 2.209 & & \\
Ca I & 2.261 & $2.2580-2.2690$ & $2.2510,2.2580,2.2705,2.2760$ \\
& 2.263 & & \\
& 2.265 & & \\
\hline
\end{tabular}

1000 times and the adopted EW uncertainties of the $\mathrm{Na}$ I and $\mathrm{Ca}$ I features for each star correspond to the standard deviations of the $1000 \mathrm{EW}$ measurements of each feature.

The values of the $\mathrm{Na}$ I and $\mathrm{Ca}$ I EWs measured for each star, along with their errors, are presented in Table 3.

\section{2. $\mathrm{H}_{2} \mathrm{O}-\mathrm{K} 2$ Index}

In RA10, the $\mathrm{H}_{2} \mathrm{O}-\mathrm{K}$ index by Covey et al. (2010) was used to account for the influence of temperature on the strengths of the $\mathrm{Na}$ and $\mathrm{Ca}$ lines being used to diagnose each star's metallicity. Covey et al. (2010) adopted this index to characterize the spectral types of highly reddened young stars from moderate $\mathrm{S} / \mathrm{N}$ spectra, and optimized the $\mathrm{H}_{2} \mathrm{O}-\mathrm{K}$ index to sample the redder portion of the $K$ band and ignored weak atomic features (Mg I, Ti I) that were not typically visible in their spectra. Stars in our sample, by contrast, possess low extinctions and are relatively bright, such that two of the regions used to calculate the $\mathrm{H}_{2} \mathrm{O}-\mathrm{K}$ index show obvious atomic absorption features in our high-S/N spectra of early $\mathrm{M}$ dwarfs. To ensure our waterindex measurements are not affected or biased by these features, we developed a modified $\mathrm{H}_{2} \mathrm{O}$-index using two new regions that do not show any noticeable atomic lines. This $\mathrm{H}_{2} \mathrm{O}-\mathrm{K} 2$ water index is defined as

$$
\mathrm{H}_{2} \mathrm{O}-\mathrm{K} 2=\frac{\langle\mathcal{F}(2.070-2.090)\rangle /\langle\mathcal{F}(2.235-2.255)\rangle}{\langle\mathcal{F}(2.235-2.255)\rangle /\langle\mathcal{F}(2.360-2.380)\rangle},
$$

where $\langle\mathcal{F}(a-b)\rangle$ denotes the median flux level in the wavelength range defined by $a$ and $b$ in $\mu \mathrm{m}$. This new index represents the change in the overall shape of the spectra of $\mathrm{M}$ dwarfs due to water absorption from $2.07 \mu \mathrm{m}$ to $2.38 \mu \mathrm{m}$, with smaller values of the $\mathrm{H}_{2} \mathrm{O}-\mathrm{K} 2$ index corresponding to greater amounts of $\mathrm{H}_{2} \mathrm{O}$ opacity. The difference between the $\mathrm{H}_{2} \mathrm{O}-\mathrm{K}$ index defined by Covey et al. (2010) and the $\mathrm{H}_{2} \mathrm{O}-\mathrm{K} 2$ index is shown in Figure 2. Uncertainties in the $\mathrm{H}_{2} \mathrm{O}-\mathrm{K} 2$ index measurements were computed using the same Monte Carlo approach used to estimate uncertainties in the $\mathrm{Na}$ I and $\mathrm{Ca}$ I lines; each $\mathrm{H}_{2} \mathrm{O}-\mathrm{K} 2$ error estimate represents the standard deviation of $1000 \mathrm{H}_{2} \mathrm{O}-\mathrm{K} 2$ measurements after adding synthetic noise to the spectrum consistent with the $\mathrm{S} / \mathrm{N}$ value of the observed spectrum.

The values of the $\mathrm{H}_{2} \mathrm{O}-\mathrm{K} 2$ index measured for each star are included in Table 3 along with their uncertainties.

\subsection{Expectations from Theoretical Models}

The metallicity calibration presented in RA10 was empirically derived, with the temperature and metallicity dependences of the $\mathrm{Na}$ I, $\mathrm{Ca}$ I, and $\mathrm{H}_{2} \mathrm{O}$ features inferred largely from visual inspection of the calibrator stars. While RA10 used PHOENIX model atmospheres to qualitatively demonstrate the metallicity sensitivity of $\mathrm{Na}$ I and $\mathrm{Ca}$ I, and the reduced metallicity sensitivity of the $\mathrm{H}_{2} \mathrm{O}$ feature, no detailed exploration of the behavior of the $\mathrm{Na}$ I, $\mathrm{Ca}$ I, and $\mathrm{H}_{2} \mathrm{O}$ features as functions of temperature or metallicity was performed.

The lack of a quantitative exploration of the line strengths predicted by model atmospheres is partially due to the fact that, until recently, synthetic models struggled to match the infrared spectrum of $\mathrm{M}$ dwarfs, which is predominately dominated by water opacity (Allard \& Hauschildt 1995). The discrepancy was believed to be due to incomplete water vapor lists; several water opacity profiles were used through the years, all of which overpredicted the $K$-band opacity and resulted in a lack of flux in the synthetic model when compared to observed spectra (Allard et al. 2010). Asplund et al. (2009) recently derived a 
Table 3

TripleSpec Nearby M Dwarf Sample—Spectral Measurements

\begin{tabular}{|c|c|c|c|c|c|c|c|}
\hline Name & $\mathrm{EW}(\mathrm{Na} \mathrm{I})$ & $\mathrm{EW}(\mathrm{Ca} \mathrm{I})$ & $\mathrm{H}_{2} \mathrm{O}-\mathrm{K} 2$ & Sp. Type & $T_{\text {eff }}$ & {$[\mathrm{M} / \mathrm{H}]$} & {$[\mathrm{Fe} / \mathrm{H}]$} \\
\hline LHS 3576 & $4.355 \pm 0.107$ & $3.883 \pm 0.095$ & $1.021 \pm 0.004$ & 0 & $3883 \pm 24$ & $-0.13 \pm 0.12$ & $-0.19 \pm 0.17$ \\
\hline Gl $338 \mathrm{~A}$ & $4.045 \pm 0.082$ & $4.358 \pm 0.081$ & $1.038 \pm 0.003$ & 0 & $4031 \pm 56$ & $-0.13 \pm 0.12$ & $-0.18 \pm 0.17$ \\
\hline Gl 338 B & $4.023 \pm 0.119$ & $4.475 \pm 0.107$ & $1.019 \pm 0.004$ & 0 & $3869 \pm 15$ & $-0.11 \pm 0.12$ & $-0.15 \pm 0.17$ \\
\hline G 210-45 & $4.998 \pm 0.104$ & $4.633 \pm 0.097$ & $1.024 \pm 0.004$ & 0 & $3910 \pm 37$ & $-0.03 \pm 0.12$ & $-0.05 \pm 0.17$ \\
\hline Gl 205 & $7.636 \pm 0.043$ & $6.105 \pm 0.043$ & $1.032 \pm 0.002$ & 0 & $4012 \pm 106$ & $0.25 \pm 0.12$ & $0.35 \pm 0.17$ \\
\hline G1 $725 \mathrm{~A}$ & $3.068 \pm 0.034$ & $2.152 \pm 0.031$ & $0.982 \pm 0.002$ & 1 & $3680 \pm 18$ & $-0.34 \pm 0.12$ & $-0.49 \pm 0.17$ \\
\hline Gl $412 \mathrm{~A}$ & $3.431 \pm 0.091$ & $2.618 \pm 0.085$ & $0.983 \pm 0.003$ & 1 & $3684 \pm 20$ & $-0.28 \pm 0.12$ & $-0.40 \pm 0.17$ \\
\hline Gl 686 & $3.855 \pm 0.039$ & $3.268 \pm 0.037$ & $0.985 \pm 0.002$ & 1 & $3693 \pm 20$ & $-0.20 \pm 0.12$ & $-0.28 \pm 0.17$ \\
\hline Gl $752 \mathrm{AB}$ & $5.626 \pm 0.074$ & $4.018 \pm 0.068$ & $1.003 \pm 0.003$ & 1 & $3789 \pm 20$ & $-0.03 \pm 0.12$ & $-0.05 \pm 0.17$ \\
\hline Gl 649 & $4.966 \pm 0.087$ & $4.534 \pm 0.033$ & $0.993 \pm 0.002$ & 1 & $3733 \pm 20$ & $-0.02 \pm 0.12$ & $-0.04 \pm 0.17$ \\
\hline HIP 12961 & $5.175 \pm 0.105$ & $4.963 \pm 0.105$ & $1.014 \pm 0.004$ & 1 & $3838 \pm 19$ & $0.01 \pm 0.12$ & $0.01 \pm 0.17$ \\
\hline Gl 212 & $5.078 \pm 0.045$ & $5.206 \pm 0.045$ & $1.016 \pm 0.002$ & 1 & $3851 \pm 17$ & $0.02 \pm 0.12$ & $0.03 \pm 0.17$ \\
\hline HD 46375 B & $7.136 \pm 0.035$ & $5.407 \pm 0.034$ & $0.978 \pm 0.002$ & 1 & $3663 \pm 15$ & $0.21 \pm 0.12$ & $0.29 \pm 0.17$ \\
\hline HIP 79431 & $8.948 \pm 0.041$ & $5.436 \pm 0.039$ & $0.984 \pm 0.002$ & 1 & $3689 \pm 20$ & $0.33 \pm 0.12$ & $0.46 \pm 0.17$ \\
\hline V* V1513 Cyg & $1.508 \pm 0.061$ & $1.964 \pm 0.059$ & $0.935 \pm 0.002$ & 2 & $3483 \pm 17$ & $-0.45 \pm 0.12$ & $-0.64 \pm 0.17$ \\
\hline Gl 411 & $3.115 \pm 0.065$ & $2.623 \pm 0.059$ & $0.945 \pm 0.002$ & 2 & $3526 \pm 18$ & $-0.28 \pm 0.12$ & $-0.41 \pm 0.17$ \\
\hline G1 526 & $3.646 \pm 0.015$ & $3.207 \pm 0.014$ & $0.973 \pm 0.001$ & 2 & $3642 \pm 17$ & $-0.21 \pm 0.12$ & $-0.30 \pm 0.17$ \\
\hline V* V547 Cas & $3.603 \pm 0.075$ & $3.246 \pm 0.068$ & $0.945 \pm 0.003$ & 2 & $3526 \pm 18$ & $-0.19 \pm 0.12$ & $-0.28 \pm 0.17$ \\
\hline Gl 872 B & $4.101 \pm 0.084$ & $3.173 \pm 0.075$ & $0.955 \pm 0.003$ & 2 & $3569 \pm 20$ & $-0.17 \pm 0.12$ & $-0.25 \pm 0.17$ \\
\hline G1 797 B & $4.298 \pm 0.054$ & $3.182 \pm 0.045$ & $0.955 \pm 0.002$ & 2 & $3569 \pm 20$ & $-0.16 \pm 0.12$ & $-0.23 \pm 0.17$ \\
\hline LHS 3577 & $4.704 \pm 0.095$ & $3.919 \pm 0.085$ & $0.968 \pm 0.003$ & 2 & $3621 \pm 19$ & $-0.07 \pm 0.12$ & $-0.11 \pm 0.17$ \\
\hline Gl 581 & $4.965 \pm 0.078$ & $3.656 \pm 0.077$ & $0.947 \pm 0.003$ & 2 & $3534 \pm 18$ & $-0.06 \pm 0.12$ & $-0.10 \pm 0.17$ \\
\hline Gl 408 & $4.755 \pm 0.015$ & $3.895 \pm 0.013$ & $0.945 \pm 0.001$ & 2 & $3526 \pm 18$ & $-0.06 \pm 0.12$ & $-0.09 \pm 0.17$ \\
\hline Gl 251 & $4.935 \pm 0.047$ & $3.862 \pm 0.053$ & $0.939 \pm 0.002$ & 2 & $3500 \pm 17$ & $-0.04 \pm 0.12$ & $-0.07 \pm 0.17$ \\
\hline Gl 297.2 B & $5.154 \pm 0.107$ & $4.055 \pm 0.125$ & $0.959 \pm 0.003$ & 2 & $3585 \pm 20$ & $-0.03 \pm 0.12$ & $-0.04 \pm 0.17$ \\
\hline G1 250 B & $5.124 \pm 0.039$ & $4.427 \pm 0.041$ & $0.955 \pm 0.002$ & 2 & $3569 \pm 20$ & $0.01 \pm 0.12$ & $0.01 \pm 0.17$ \\
\hline Gl 176 & $6.155 \pm 0.042$ & $4.806 \pm 0.042$ & $0.958 \pm 0.002$ & 2 & $3581 \pm 20$ & $0.11 \pm 0.12$ & $0.15 \pm 0.17$ \\
\hline NLTT 14186 & $6.259 \pm 0.047$ & $5.302 \pm 0.041$ & $0.946 \pm 0.002$ & 2 & $3530 \pm 18$ & $0.17 \pm 0.12$ & $0.24 \pm 0.17$ \\
\hline G1 849 & $7.198 \pm 0.055$ & $5.382 \pm 0.049$ & $0.963 \pm 0.002$ & 2 & $3601 \pm 19$ & $0.23 \pm 0.12$ & $0.31 \pm 0.17$ \\
\hline Gl 725 B & $3.594 \pm 0.035$ & $2.308 \pm 0.033$ & $0.895 \pm 0.002$ & 3 & $3288 \pm 27$ & $-0.25 \pm 0.12$ & $-0.36 \pm 0.17$ \\
\hline Gl $661 \mathrm{AB}$ & $3.577 \pm 0.039$ & $2.714 \pm 0.035$ & $0.892 \pm 0.002$ & 3 & $3272 \pm 28$ & $-0.21 \pm 0.12$ & $-0.31 \pm 0.17$ \\
\hline G 262-29 & $3.474 \pm 0.072$ & $3.056 \pm 0.065$ & $0.929 \pm 0.002$ & 3 & $3455 \pm 16$ & $-0.21 \pm 0.12$ & $-0.30 \pm 0.17$ \\
\hline LHS 3605 & $3.946 \pm 0.098$ & $2.879 \pm 0.086$ & $0.925 \pm 0.003$ & 3 & $3437 \pm 16$ & $-0.19 \pm 0.12$ & $-0.28 \pm 0.17$ \\
\hline LHS 115 & $4.101 \pm 0.085$ & $2.683 \pm 0.078$ & $0.905 \pm 0.004$ & 3 & $3339 \pm 23$ & $-0.19 \pm 0.12$ & $-0.27 \pm 0.17$ \\
\hline Gl 625 & $4.112 \pm 0.049$ & $3.144 \pm 0.046$ & $0.931 \pm 0.002$ & 3 & $3464 \pm 17$ & $-0.16 \pm 0.12$ & $-0.23 \pm 0.17$ \\
\hline Gl 643 & $4.703 \pm 0.055$ & $2.617 \pm 0.052$ & $0.912 \pm 0.002$ & 3 & $3376 \pm 20$ & $-0.15 \pm 0.12$ & $-0.22 \pm 0.17$ \\
\hline Gl 273 & $4.667 \pm 0.085$ & $2.915 \pm 0.104$ & $0.896 \pm 0.003$ & 3 & $3293 \pm 26$ & $-0.12 \pm 0.12$ & $-0.17 \pm 0.17$ \\
\hline LHS 3591 & $4.777 \pm 0.104$ & $3.416 \pm 0.092$ & $0.923 \pm 0.003$ & 3 & $3428 \pm 16$ & $-0.08 \pm 0.12$ & $-0.12 \pm 0.17$ \\
\hline Gl $860 \mathrm{AB}$ & $5.075 \pm 0.097$ & $3.348 \pm 0.088$ & $0.931 \pm 0.003$ & 3 & $3464 \pm 17$ & $-0.07 \pm 0.12$ & $-0.11 \pm 0.17$ \\
\hline Gl 687 & $4.643 \pm 0.057$ & $3.712 \pm 0.027$ & $0.916 \pm 0.002$ & 3 & $3395 \pm 18$ & $-0.06 \pm 0.12$ & $-0.09 \pm 0.17$ \\
\hline Gl 628 & $5.548 \pm 0.036$ & $3.508 \pm 0.034$ & $0.913 \pm 0.002$ & 3 & $3380 \pm 20$ & $-0.01 \pm 0.12$ & $-0.02 \pm 0.17$ \\
\hline Gl 873 & $5.573 \pm 0.072$ & $3.642 \pm 0.068$ & $0.917 \pm 0.003$ & 3 & $3400 \pm 18$ & $-0.00 \pm 0.12$ & $-0.01 \pm 0.17$ \\
\hline LHS 3558 & $4.927 \pm 0.048$ & $4.312 \pm 0.042$ & $0.925 \pm 0.002$ & 3 & $3437 \pm 16$ & $0.01 \pm 0.12$ & $0.01 \pm 0.17$ \\
\hline G 168-24 & $5.266 \pm 0.043$ & $4.029 \pm 0.037$ & $0.923 \pm 0.002$ & 3 & $3428 \pm 16$ & $0.01 \pm 0.12$ & $0.01 \pm 0.17$ \\
\hline HD 222582 B & $5.622 \pm 0.076$ & $3.607 \pm 0.075$ & $0.895 \pm 0.003$ & 3 & $3288 \pm 27$ & $0.02 \pm 0.12$ & $0.02 \pm 0.17$ \\
\hline Gl 436 & $5.373 \pm 0.029$ & $4.302 \pm 0.024$ & $0.932 \pm 0.002$ & 3 & $3469 \pm 17$ & $0.03 \pm 0.12$ & $0.04 \pm 0.17$ \\
\hline HIP 57050 & $5.659 \pm 0.053$ & $3.806 \pm 0.051$ & $0.895 \pm 0.002$ & 3 & $3288 \pm 27$ & $0.04 \pm 0.12$ & $0.05 \pm 0.17$ \\
\hline LP 816-60 & $6.185 \pm 0.034$ & $3.725 \pm 0.031$ & $0.918 \pm 0.002$ & 3 & $3405 \pm 17$ & $0.05 \pm 0.12$ & $0.06 \pm 0.17$ \\
\hline G1 896 A & $5.861 \pm 0.032$ & $4.515 \pm 0.026$ & $0.904 \pm 0.002$ & 3 & $3334 \pm 23$ & $0.11 \pm 0.12$ & $0.15 \pm 0.17$ \\
\hline Gl 876 & $6.777 \pm 0.049$ & $4.363 \pm 0.047$ & $0.933 \pm 0.002$ & 3 & $3473 \pm 17$ & $0.14 \pm 0.12$ & $0.19 \pm 0.17$ \\
\hline Gl 402 & $7.144 \pm 0.021$ & $3.882 \pm 0.017$ & $0.904 \pm 0.001$ & 3 & $3334 \pm 23$ & $0.15 \pm 0.12$ & $0.20 \pm 0.17$ \\
\hline Gl 53.1 B & $7.006 \pm 0.055$ & $3.954 \pm 0.055$ & $0.894 \pm 0.002$ & 3 & $3282 \pm 27$ & $0.15 \pm 0.12$ & $0.21 \pm 0.17$ \\
\hline Gl 555 & $7.051 \pm 0.041$ & $3.992 \pm 0.039$ & $0.895 \pm 0.002$ & 3 & $3288 \pm 27$ & $0.16 \pm 0.12$ & $0.22 \pm 0.17$ \\
\hline Gl 179 & $6.793 \pm 0.105$ & $4.554 \pm 0.098$ & $0.922 \pm 0.003$ & 3 & $3424 \pm 16$ & $0.17 \pm 0.12$ & $0.23 \pm 0.17$ \\
\hline LHS 494 & $6.477 \pm 0.061$ & $4.619 \pm 0.054$ & $0.895 \pm 0.002$ & 3 & $3288 \pm 27$ & $0.17 \pm 0.12$ & $0.24 \pm 0.17$ \\
\hline Gl 388 & $6.523 \pm 0.107$ & $5.105 \pm 0.098$ & $0.915 \pm 0.003$ & 3 & $3390 \pm 19$ & $0.20 \pm 0.12$ & $0.28 \pm 0.17$ \\
\hline Gl $169.1 \mathrm{~A}$ & $8.323 \pm 0.019$ & $4.058 \pm 0.018$ & $0.892 \pm 0.001$ & 3 & $3272 \pm 28$ & $0.26 \pm 0.12$ & $0.36 \pm 0.17$ \\
\hline LHS 3409 & $3.254 \pm 0.235$ & $1.313 \pm 0.109$ & $0.875 \pm 0.003$ & 4 & $3178 \pm 41$ & $-0.36 \pm 0.12$ & $-0.52 \pm 0.18$ \\
\hline Gl 699 & $3.745 \pm 0.043$ & $1.967 \pm 0.037$ & $0.891 \pm 0.002$ & 4 & $3266 \pm 29$ & $-0.27 \pm 0.12$ & $-0.39 \pm 0.17$ \\
\hline LHS 220 & $3.905 \pm 0.094$ & $1.966 \pm 0.091$ & $0.863 \pm 0.003$ & 4 & $3093 \pm 70$ & $-0.24 \pm 0.12$ & $-0.35 \pm 0.17$ \\
\hline G1 783.2 B & $4.032 \pm 0.053$ & $2.365 \pm 0.044$ & $0.875 \pm 0.002$ & 4 & $3178 \pm 41$ & $-0.20 \pm 0.12$ & $-0.29 \pm 0.17$ \\
\hline Gl 445 & $3.884 \pm 0.145$ & $2.825 \pm 0.135$ & $0.878 \pm 0.004$ & 4 & $3195 \pm 38$ & $-0.17 \pm 0.12$ & $-0.25 \pm 0.17$ \\
\hline Gl 213 & $4.358 \pm 0.088$ & $2.443 \pm 0.086$ & $0.874 \pm 0.003$ & 4 & $3167 \pm 47$ & $-0.17 \pm 0.12$ & $-0.25 \pm 0.17$ \\
\hline Gl 544 B & $5.263 \pm 0.037$ & $2.748 \pm 0.038$ & $0.851 \pm 0.002$ & 4 & $3058 \pm 65$ & $-0.05 \pm 0.12$ & $-0.09 \pm 0.17$ \\
\hline LHS 1723 & $5.463 \pm 0.051$ & $2.837 \pm 0.047$ & $0.855 \pm 0.002$ & 4 & $3054 \pm 69$ & $-0.03 \pm 0.12$ & $-0.06 \pm 0.17$ \\
\hline GJ 1224 & $6.032 \pm 0.035$ & $2.702 \pm 0.033$ & $0.887 \pm 0.002$ & 4 & $3245 \pm 31$ & $-0.03 \pm 0.12$ & $-0.05 \pm 0.17$ \\
\hline GJ 1119 & $5.599 \pm 0.032$ & $3.091 \pm 0.028$ & $0.882 \pm 0.002$ & 4 & $3218 \pm 34$ & $-0.02 \pm 0.12$ & $-0.04 \pm 0.17$ \\
\hline G 041-014 & $5.324 \pm 0.033$ & $3.309 \pm 0.031$ & $0.875 \pm 0.002$ & 4 & $3178 \pm 41$ & $-0.02 \pm 0.12$ & $-0.03 \pm 0.17$ \\
\hline GJ 3348 B & $5.438 \pm 0.073$ & $3.323 \pm 0.064$ & $0.877 \pm 0.002$ & 4 & $3189 \pm 39$ & $-0.01 \pm 0.12$ & $-0.02 \pm 0.17$ \\
\hline
\end{tabular}


Table 3

(Continued)

\begin{tabular}{|c|c|c|c|c|c|c|c|}
\hline Name & EW(Na I) & $\mathrm{EW}(\mathrm{Ca} \mathrm{I})$ & $\mathrm{H}_{2} \mathrm{O}-\mathrm{K} 2$ & Sp.T. & $T_{\text {eff }}$ & {$[\mathrm{M} / \mathrm{H}]$} & {$[\mathrm{Fe} / \mathrm{H}]$} \\
\hline LHS 1809 & $6.476 \pm 0.037$ & $2.359 \pm 0.036$ & $0.855 \pm 0.002$ & 4 & $3054 \pm 69$ & $-0.00 \pm 0.12$ & $-0.01 \pm 0.17$ \\
\hline Gl 447 & $5.655 \pm 0.092$ & $3.006 \pm 0.088$ & $0.853 \pm 0.003$ & 4 & $3065 \pm 69$ & $-0.00 \pm 0.12$ & $-0.01 \pm 0.17$ \\
\hline LHS 1066 & $5.459 \pm 0.049$ & $3.244 \pm 0.048$ & $0.851 \pm 0.002$ & 4 & $3058 \pm 65$ & $0.01 \pm 0.12$ & $0.00 \pm 0.17$ \\
\hline GJ 3134 & $5.562 \pm 0.058$ & $3.274 \pm 0.055$ & $0.861 \pm 0.002$ & 4 & $3080 \pm 74$ & $0.01 \pm 0.12$ & $0.01 \pm 0.17$ \\
\hline Gl 231.1 B & $5.602 \pm 0.034$ & $3.458 \pm 0.032$ & $0.877 \pm 0.002$ & 4 & $3189 \pm 39$ & $0.02 \pm 0.12$ & $0.02 \pm 0.17$ \\
\hline LHS 3593 & $6.126 \pm 0.125$ & $3.095 \pm 0.123$ & $0.868 \pm 0.004$ & 4 & $3129 \pm 58$ & $0.03 \pm 0.12$ & $0.03 \pm 0.17$ \\
\hline GJ 3379 & $6.106 \pm 0.042$ & $3.502 \pm 0.045$ & $0.875 \pm 0.002$ & 4 & $3178 \pm 41$ & $0.06 \pm 0.12$ & $0.08 \pm 0.17$ \\
\hline Gl $268 \mathrm{AB}$ & $6.753 \pm 0.047$ & $3.175 \pm 0.054$ & $0.876 \pm 0.002$ & 4 & $3184 \pm 40$ & $0.08 \pm 0.12$ & $0.10 \pm 0.17$ \\
\hline G1 768.1 B & $5.847 \pm 0.056$ & $4.005 \pm 0.051$ & $0.887 \pm 0.002$ & 4 & $3245 \pm 31$ & $0.08 \pm 0.12$ & $0.10 \pm 0.17$ \\
\hline NLTT 25869 & $6.452 \pm 0.051$ & $3.229 \pm 0.049$ & $0.853 \pm 0.002$ & 4 & $3065 \pm 69$ & $0.08 \pm 0.12$ & $0.11 \pm 0.17$ \\
\hline LHS 6007 & $6.546 \pm 0.062$ & $3.951 \pm 0.062$ & $0.891 \pm 0.002$ & 4 & $3266 \pm 29$ & $0.12 \pm 0.12$ & $0.16 \pm 0.17$ \\
\hline Gl 905 & $7.796 \pm 0.035$ & $2.732 \pm 0.035$ & $0.851 \pm 0.002$ & 4 & $3058 \pm 65$ & $0.14 \pm 0.12$ & $0.19 \pm 0.17$ \\
\hline LHS 495 & $7.259 \pm 0.115$ & $3.354 \pm 0.104$ & $0.861 \pm 0.003$ & 4 & $3080 \pm 74$ & $0.15 \pm 0.12$ & $0.20 \pm 0.17$ \\
\hline GJ 1214 & $7.753 \pm 0.096$ & $3.275 \pm 0.067$ & $0.887 \pm 0.003$ & 4 & $3245 \pm 31$ & $0.15 \pm 0.12$ & $0.20 \pm 0.17$ \\
\hline G 246-33 & $6.468 \pm 0.051$ & $4.232 \pm 0.052$ & $0.861 \pm 0.002$ & 4 & $3080 \pm 74$ & $0.17 \pm 0.12$ & $0.24 \pm 0.17$ \\
\hline Gl 324 B & $7.818 \pm 0.035$ & $4.019 \pm 0.034$ & $0.889 \pm 0.002$ & 4 & $3256 \pm 29$ & $0.22 \pm 0.12$ & $0.31 \pm 0.17$ \\
\hline G 203-47 & $7.978 \pm 0.045$ & $3.969 \pm 0.043$ & $0.882 \pm 0.002$ & 4 & $3218 \pm 34$ & $0.24 \pm 0.12$ & $0.33 \pm 0.17$ \\
\hline Gl 299 & $3.354 \pm 0.035$ & $1.456 \pm 0.032$ & $0.839 \pm 0.002$ & 5 & $3021 \pm 49$ & $-0.32 \pm 0.12$ & $-0.46 \pm 0.17$ \\
\hline LHS 224 & $2.905 \pm 0.063$ & $1.762 \pm 0.056$ & $0.826 \pm 0.002$ & 5 & $2982 \pm 34$ & $-0.32 \pm 0.12$ & $-0.46 \pm 0.17$ \\
\hline Gl 611 B & $3.277 \pm 0.037$ & $1.635 \pm 0.029$ & $0.849 \pm 0.002$ & 5 & $3051 \pm 61$ & $-0.32 \pm 0.12$ & $-0.45 \pm 0.17$ \\
\hline Gl $630.1 \mathrm{~A}$ & $3.295 \pm 0.071$ & $1.978 \pm 0.067$ & $0.829 \pm 0.002$ & 5 & $2991 \pm 37$ & $-0.27 \pm 0.12$ & $-0.39 \pm 0.17$ \\
\hline NSV 13261 & $4.375 \pm 0.075$ & $1.707 \pm 0.075$ & $0.812 \pm 0.003$ & 5 & $2939 \pm 20$ & $-0.20 \pm 0.12$ & $-0.29 \pm 0.17$ \\
\hline NLTT 15867 & $4.513 \pm 0.087$ & $2.242 \pm 0.086$ & $0.821 \pm 0.003$ & 5 & $2967 \pm 28$ & $-0.14 \pm 0.12$ & $-0.21 \pm 0.17$ \\
\hline G1 $166 \mathrm{C}$ & $4.522 \pm 0.046$ & $2.658 \pm 0.049$ & $0.825 \pm 0.002$ & 5 & $2979 \pm 32$ & $-0.10 \pm 0.12$ & $-0.15 \pm 0.17$ \\
\hline LHS 3376 & $5.288 \pm 0.042$ & $2.333 \pm 0.039$ & $0.836 \pm 0.002$ & 5 & $3012 \pm 45$ & $-0.08 \pm 0.12$ & $-0.12 \pm 0.17$ \\
\hline GJ 3253 & $5.315 \pm 0.068$ & $2.723 \pm 0.065$ & $0.842 \pm 0.003$ & 5 & $3030 \pm 52$ & $-0.05 \pm 0.12$ & $-0.07 \pm 0.17$ \\
\hline GJ 3069 & $5.675 \pm 0.069$ & $2.448 \pm 0.065$ & $0.839 \pm 0.003$ & 5 & $3021 \pm 49$ & $-0.04 \pm 0.12$ & $-0.07 \pm 0.17$ \\
\hline GJ 1286 & $6.408 \pm 0.126$ & $2.083 \pm 0.129$ & $0.836 \pm 0.004$ & 5 & $3012 \pm 45$ & $-0.02 \pm 0.12$ & $-0.04 \pm 0.17$ \\
\hline Gl 164 & $5.956 \pm 0.055$ & $2.927 \pm 0.053$ & $0.849 \pm 0.002$ & 5 & $3051 \pm 61$ & $0.02 \pm 0.12$ & $0.02 \pm 0.17$ \\
\hline G1 777 B & $5.857 \pm 0.055$ & $3.005 \pm 0.048$ & $0.839 \pm 0.002$ & 5 & $3021 \pm 49$ & $0.03 \pm 0.12$ & $0.03 \pm 0.17$ \\
\hline Gl 866 & $6.938 \pm 0.071$ & $2.085 \pm 0.065$ & $0.816 \pm 0.002$ & 5 & $2952 \pm 23$ & $0.04 \pm 0.12$ & $0.05 \pm 0.17$ \\
\hline Gl $473 \mathrm{AB}$ & $6.368 \pm 0.072$ & $2.652 \pm 0.085$ & $0.825 \pm 0.003$ & 5 & $2979 \pm 32$ & $0.05 \pm 0.12$ & $0.05 \pm 0.17$ \\
\hline Gl $234 \mathrm{AB}$ & $6.419 \pm 0.038$ & $3.348 \pm 0.039$ & $0.845 \pm 0.002$ & 5 & $3039 \pm 56$ & $0.10 \pm 0.12$ & $0.13 \pm 0.17$ \\
\hline LHS 3549 & $7.097 \pm 0.087$ & $2.958 \pm 0.059$ & $0.836 \pm 0.002$ & 5 & $3012 \pm 45$ & $0.12 \pm 0.12$ & $0.16 \pm 0.17$ \\
\hline LHS 1706 & $7.681 \pm 0.075$ & $2.822 \pm 0.079$ & $0.834 \pm 0.003$ & 5 & $3006 \pm 43$ & $0.16 \pm 0.12$ & $0.21 \pm 0.17$ \\
\hline $\mathrm{V} * \mathrm{~V} 388 \mathrm{Cas}$ & $7.326 \pm 0.067$ & $3.737 \pm 0.066$ & $0.845 \pm 0.002$ & 5 & $3039 \pm 56$ & $0.21 \pm 0.12$ & $0.28 \pm 0.17$ \\
\hline LHS 3799 & $8.478 \pm 0.075$ & $3.508 \pm 0.068$ & $0.845 \pm 0.003$ & 5 & $3039 \pm 56$ & $0.28 \pm 0.12$ & $0.38 \pm 0.17$ \\
\hline Gl 285 & $7.343 \pm 0.019$ & $4.545 \pm 0.019$ & $0.847 \pm 0.002$ & 5 & $3045 \pm 59$ & $0.29 \pm 0.12$ & $0.40 \pm 0.17$ \\
\hline LHS 18 & $3.285 \pm 0.095$ & $1.213 \pm 0.071$ & $0.802 \pm 0.002$ & 6 & $2909 \pm 17$ & $-0.34 \pm 0.12$ & $-0.48 \pm 0.17$ \\
\hline Gl 412 B & $5.138 \pm 0.076$ & $0.385 \pm 0.074$ & $0.804 \pm 0.003$ & 6 & $2915 \pm 18$ & $-0.27 \pm 0.12$ & $-0.39 \pm 0.17$ \\
\hline LHS 1901 & $4.322 \pm 0.054$ & $1.231 \pm 0.053$ & $0.775 \pm 0.003$ & 6 & $2826 \pm 21$ & $-0.23 \pm 0.12$ & $-0.34 \pm 0.17$ \\
\hline LSPM J0011 $^{\mathrm{a}}$ & $5.109 \pm 0.049$ & $1.285 \pm 0.051$ & $0.805 \pm 0.003$ & 6 & $2918 \pm 18$ & $-0.18 \pm 0.12$ & $-0.27 \pm 0.17$ \\
\hline GJ $1245 \mathrm{AC}$ & $5.595 \pm 0.092$ & $1.675 \pm 0.108$ & $0.795 \pm 0.002$ & 6 & $2890 \pm 19$ & $-0.09 \pm 0.12$ & $-0.14 \pm 0.17$ \\
\hline GJ 1245 B & $5.695 \pm 0.068$ & $1.615 \pm 0.065$ & $0.792 \pm 0.003$ & 6 & $2881 \pm 20$ & $-0.09 \pm 0.12$ & $-0.13 \pm 0.17$ \\
\hline GJ $1116 \mathrm{AB}$ & $5.735 \pm 0.028$ & $1.709 \pm 0.025$ & $0.797 \pm 0.002$ & 6 & $2896 \pm 18$ & $-0.08 \pm 0.12$ & $-0.12 \pm 0.17$ \\
\hline GJ 3146 & $6.066 \pm 0.062$ & $1.457 \pm 0.058$ & $0.788 \pm 0.002$ & 6 & $2868 \pm 21$ & $-0.07 \pm 0.12$ & $-0.11 \pm 0.17$ \\
\hline LHS 252 & $6.004 \pm 0.036$ & $2.287 \pm 0.036$ & $0.798 \pm 0.002$ & 6 & $2898 \pm 17$ & $0.00 \pm 0.12$ & $-0.01 \pm 0.17$ \\
\hline G1 376 B & $7.725 \pm 0.077$ & $1.687 \pm 0.081$ & $0.776 \pm 0.003$ & 6 & $2829 \pm 21$ & $0.11 \pm 0.12$ & $0.14 \pm 0.17$ \\
\hline Gl 1156 & $6.645 \pm 0.058$ & $2.765 \pm 0.057$ & $0.792 \pm 0.002$ & 6 & $2881 \pm 20$ & $0.11 \pm 0.12$ & $0.15 \pm 0.17$ \\
\hline Gl 406 & $7.994 \pm 0.015$ & $1.977 \pm 0.015$ & $0.794 \pm 0.001$ & 6 & $2887 \pm 20$ & $0.14 \pm 0.12$ & $0.18 \pm 0.17$ \\
\hline GJ 3147 & $7.022 \pm 0.058$ & $2.773 \pm 0.054$ & $0.779 \pm 0.002$ & 6 & $2839 \pm 21$ & $0.16 \pm 0.12$ & $0.21 \pm 0.17$ \\
\hline LHS 292 & $4.791 \pm 0.035$ & $0.294 \pm 0.035$ & $0.759 \pm 0.002$ & 7 & $2772 \pm 25$ & $-0.28 \pm 0.12$ & $-0.41 \pm 0.17$ \\
\hline Gl $644 \mathrm{C}$ & $5.117 \pm 0.054$ & $0.571 \pm 0.051$ & $0.745 \pm 0.002$ & 7 & $2727 \pm 29$ & $-0.21 \pm 0.12$ & $-0.32 \pm 0.17$ \\
\hline GJ 1111 & $5.661 \pm 0.039$ & $1.039 \pm 0.038$ & $0.758 \pm 0.002$ & 7 & $2769 \pm 26$ & $-0.12 \pm 0.12$ & $-0.19 \pm 0.17$ \\
\hline LHS 2090 & $6.242 \pm 0.032$ & $1.405 \pm 0.032$ & $0.758 \pm 0.002$ & 7 & $2769 \pm 26$ & $-0.03 \pm 0.12$ & $-0.06 \pm 0.17$ \\
\hline V* V492 Lyr & $6.406 \pm 0.075$ & $1.225 \pm 0.078$ & $0.739 \pm 0.003$ & 7 & $2709 \pm 28$ & $-0.02 \pm 0.12$ & $-0.04 \pm 0.17$ \\
\hline Teegardens & $3.429 \pm 0.039$ & $0.269 \pm 0.032$ & $0.713 \pm 0.003$ & 8 & $2637 \pm 30$ & $-0.38 \pm 0.12$ & $-0.55 \pm 0.17$ \\
\hline 2MASS J1835 & $3.886 \pm 0.075$ & $0.721 \pm 0.073$ & $0.692 \pm 0.003$ & 8 & $2578 \pm 39$ & $-0.27 \pm 0.12$ & $-0.40 \pm 0.17$ \\
\hline LHS 2065 & $7.252 \pm 0.104$ & $1.316 \pm 0.099$ & $0.687 \pm 0.003$ & 8 & $2564 \pm 40$ & $0.12 \pm 0.12$ & $0.16 \pm 0.17$ \\
\hline LHS 2924 & $4.995 \pm 0.049$ & $0.845 \pm 0.053$ & $0.661 \pm 0.002$ & 9 & $2492 \pm 43$ & $-0.13 \pm 0.12$ & $-0.19 \pm 0.17$ \\
\hline Gl $908^{c}$ & $2.153 \pm 0.115$ & $2.275 \pm 0.113$ & $1.035 \pm 0.004$ & 0 & $3995 \pm 47$ & $-0.41 \pm 0.12$ & $-0.59 \pm 0.17$ \\
\hline Gl $809^{c}$ & $4.085 \pm 0.043$ & $4.193 \pm 0.041$ & $1.057 \pm 0.002$ & 0 & $\ldots$ & $-0.15 \pm 0.12$ & $-0.21 \pm 0.17$ \\
\hline Gl $644 \mathrm{AB}^{\mathrm{c}}$ & $3.623 \pm 0.048$ & $2.769 \pm 0.045$ & $1.015 \pm 0.003$ & 1 & $3845 \pm 19$ & $-0.27 \pm 0.12$ & $-0.39 \pm 0.17$ \\
\hline Gl $829 \mathrm{AB}^{\mathrm{c}}$ & $4.696 \pm 0.123$ & $3.265 \pm 0.129$ & $0.907 \pm 0.004$ & 3 & $3349 \pm 22$ & $-0.09 \pm 0.12$ & $-0.13 \pm 0.17$ \\
\hline
\end{tabular}

Notes.

${ }^{\text {a }}$ LSPM J0011+5908.

b 2MASS J18353790+3259545.

${ }^{\text {c }}$ Stars with low quality $K$-band spectra. 


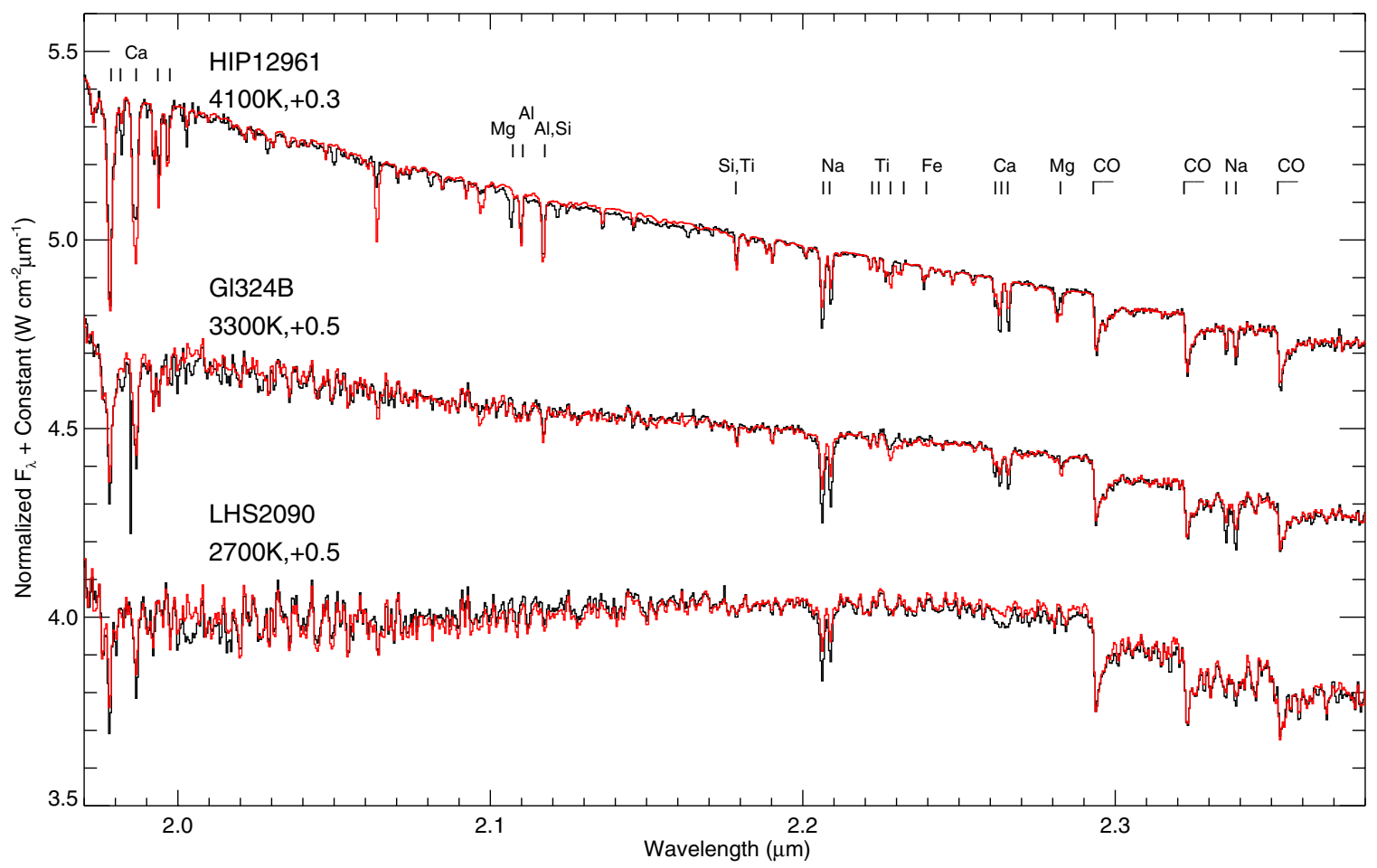

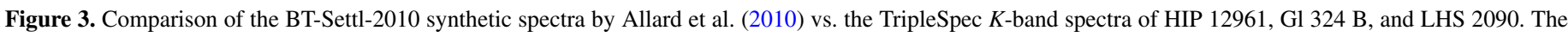

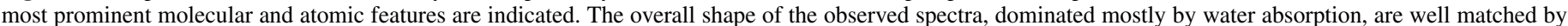

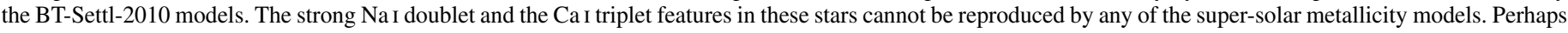

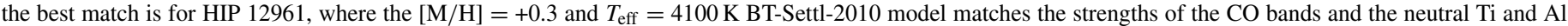

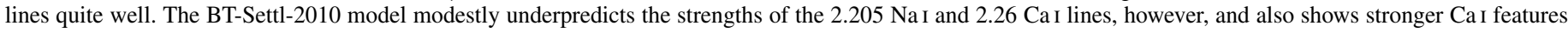
at $\sim 1.97 \mu \mathrm{m}$, and no Mg I line at $\sim 2.11 \mu \mathrm{m}$. Uncertain oscillator strengths and missing opacity sources could explain these discrepancies (Rajpurohit et al. 2010).

(A color version of this figure is available in the online journal.)

new estimate of the solar oxygen abundance, however, which is a factor of two lower than previous estimates and seems to have solved the water opacity discrepancy. The new BTSettl-2010 synthetic spectra by Allard et al. (2010) incorporate the new solar abundances, along with updated molecular line lists, and provide a better match to the spectral distribution of $\mathrm{M}$ dwarfs across the near-infrared than previous models. The BT-Settl-2010 models are available online ${ }^{11}$ using this interface, we constructed a grid of BT-Settl-2010 synthetic spectra with effective temperatures from $2200 \mathrm{~K}$ to $4100 \mathrm{~K}$, and overall metallicities $[\mathrm{M} / \mathrm{H}]$ equal to $+0.5,+0.3,+0.0,-0.5$, and -1.0 dex. This effective temperature range covers the whole $\mathrm{M}$ dwarf sequence, and the model grid covers a range of metallicities larger than that spanned by our calibration stars, or the metallicities estimated with Equation (8) for any of our target stars. All the stars analyzed here are known to be dwarfs and none show any spectral features indicating otherwise, so a value of $\log g=5.0$ was selected to provide the best agreement with empirical M dwarf gravity determinations (Fernandez et al. 2009; Demory et al. 2009).

To provide a quantitative indication of the feature strengths predicted by the theoretical models, we degraded the resolution of each theoretical spectrum to match that of our observed sample and measured the EWs of the $\mathrm{Na}$ I doublet and $\mathrm{Ca}$ I triplet, as well as the $\mathrm{H}_{2} \mathrm{O}-\mathrm{K} 2$ index, using the same routines applied to our observed spectra.

Figure 3 shows the spectra of three stars in our sample, ranging in spectral type from M1 to M7, superimposed by BT-Settl-2010 synthetic spectra degraded to the resolution of

\footnotetext{
11 http://phoenix.ens-lyon.fr/simulator
}

the TripleSpec data. The overall shape of the observed spectra, dominated mostly by water absorption, is well matched by the BT-Settl-2010 models. The Na I and $\mathrm{Ca}$ I atomic features used by the metallicity calibration fits, however, are noticeably weaker in the BT-Settl-2010 models than in the observed spectra: even the highest metallicity synthetic spectra cannot reproduce the strengths of the $\mathrm{NaI}$ doublet and $\mathrm{Ca}$ I triplet in the spectra of Gl $324 \mathrm{~B}$ and LHS 2090. The $[\mathrm{M} / \mathrm{H}]=+0.3$ and $T_{\text {eff }}=$ 4100 K BT-Settl-2010 model matches the strengths of the CO bands and the neutral Ti and Al lines for HIP 12961 quite well, but it shows stronger $\mathrm{Ca}$ I features at $\sim 1.97 \mu \mathrm{m}$, and no $\mathrm{Mg}$ I line at $\sim 2.11 \mu \mathrm{m}$. These discrepancies could be explained by uncertainties in the oscillator strengths and opacities adopted for these lines, as suggested by Rajpurohit et al. (2010). Another explanation could be the size of the wavelength interval (resolution) chosen to calculate the synthetic spectrum. If the lines in question are not well sampled at the resolution used to calculate the synthetic spectrum, convolving the synthetic spectrum with a Gaussian to degrade the resolution to match TripleSpec could cause them to be artificially "weakened."

Figure 4 shows the $\mathrm{H}_{2} \mathrm{O}-\mathrm{K} 2$ index and EWs of the $\mathrm{NaI}$ doublet and CaI triplet measured from the BT-Settl-2010 models, after degrading their spectral resolution to match that of TripleSpec, as a function of effective temperature and colorcoded by metallicity. The CaI triplet behaves as expected from observed data: its strength increases as both temperature and metallicity increase. However, the Ca I triplet disappears from the synthetic models at effective temperatures lower than $\sim 2900 \mathrm{~K}$, even for the super-solar models. In our high $\mathrm{S} / \mathrm{N}$ empirical spectra, the $\mathrm{Ca}$ I triplet remains visible even at spectral type M8. 


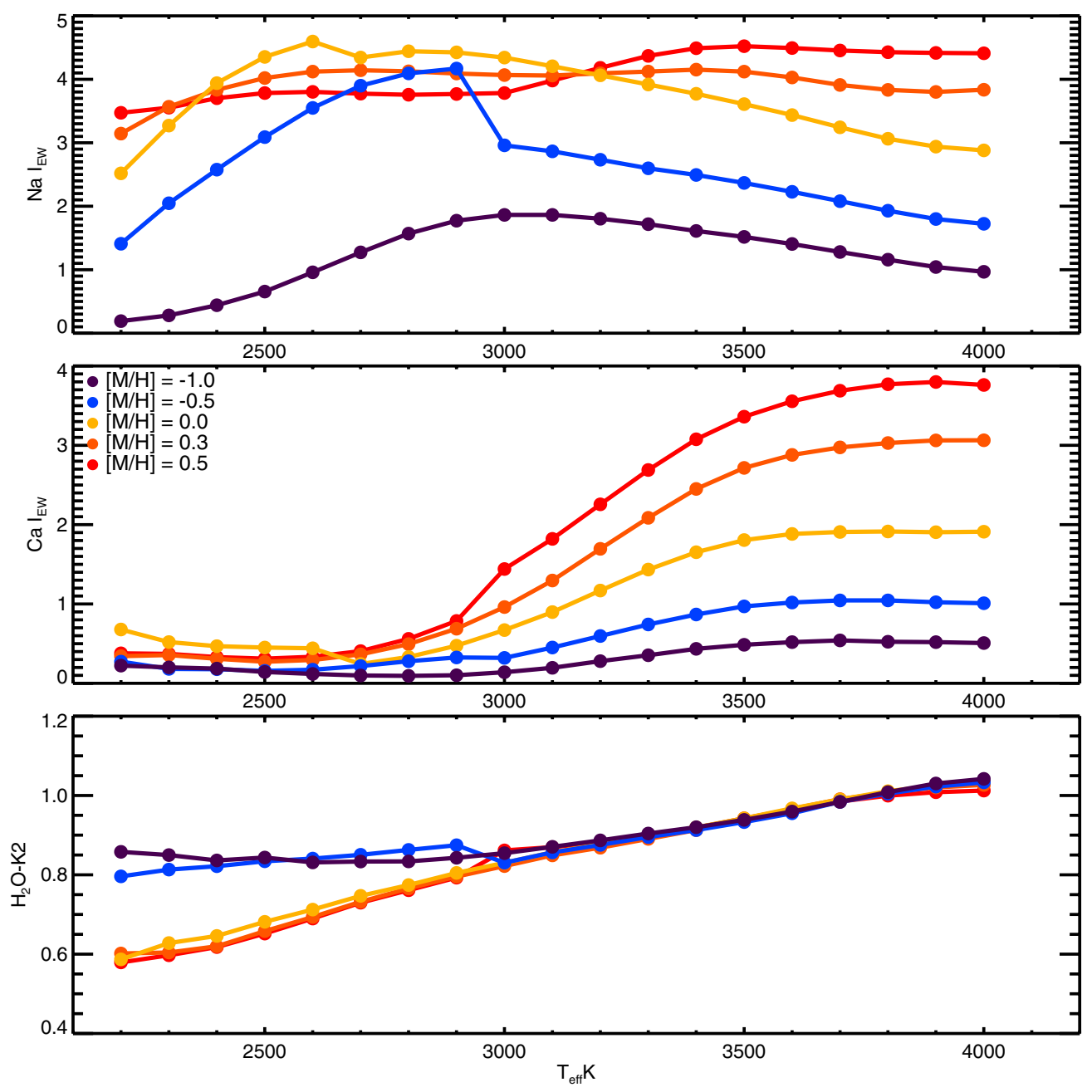

Figure 4. Equivalent widths of the NaI doublet (top) and $\mathrm{Ca}$ I triplet (middle), and $\mathrm{H}_{2} \mathrm{O}-\mathrm{K} 2$ index (bottom), measured from the BT-Settl-2010 synthetic spectra computed by Allard et al. (2010) and shown as a function of model $T_{\text {eff }}$. The strengths of the Na I and Ca I features are somewhat weaker in the synthetic spectra than in observed data, but the qualitative behavior of the CaI feature as a function of both temperature and metallicity is consistent. Water absorption appears to be a monotonic function of temperature, independent of metallicity, for models with $T_{\text {eff }} \geqslant 3000 \mathrm{~K}$. Below $3000 \mathrm{~K}$, however, the $\mathrm{H}_{2} \mathrm{O}-\mathrm{K} 2$ indices measured from the $[\mathrm{M} / \mathrm{H}] \leqslant-0.5$ dex spectral grids diverge from the $[\mathrm{M} / \mathrm{H}]>-0.5$ models; the stark divergence between the two domains suggests that the difference may be a computational artifact rather than a true astrophysical difference. The Na I doublet shows the oddest behavior in these spectra, with significant structure as a function of both metallicity and temperature.

(A color version of this figure is available in the online journal.)

The water absorption features predicted by the models also agree reasonably well with expectations from empirical spectra, with water absorption increasing monotonically with decreasing temperature, in a manner independent of metallicity, down to $T_{\text {eff }} \sim 3000 \mathrm{~K}$. Below $3000 \mathrm{~K}$, however, the models show a sharp metallicity difference: for the subsolar metallicity models, the $\mathrm{H}_{2} \mathrm{O}$ absorption remains almost constant from $3000 \mathrm{~K}$ to $2200 \mathrm{~K}$, while water absorption in solar and super-solar models continues to increase with decreasing temperature. The stark difference in the $\mathrm{H}_{2} \mathrm{O}$ opacity between solar and subsolar metallicity models suggests that the discrepancy in $\mathrm{H}_{2} \mathrm{O}$ absorption may be more likely a computational artifact than an astrophysical difference.

The most puzzling behavior is shown by the EWs for the Na I doublet. Observationally, the strength of the Na I doublet increases as temperature decreases, at least until effective temperatures equivalent to an M6V star (e.g., Cushing et al. 2005). Strong Na I doublets are also seen in the late-type stars studied in this work. The only synthetic spectra that show this trend, however, are the solar metallicity spectra. The most metal-poor $([\mathrm{M} / \mathrm{H}]=-1.0)$ synthetic spectra, by contrast, show the strongest $\mathrm{NaI}$ absorption near $\sim 3000 \mathrm{~K}$, with the
Na I EWs decreasing to both hotter and cooler temperatures; the moderately metal-poor $([\mathrm{M} / \mathrm{H}]=-0.5)$ models show a similar behavior but with a strong jump near $T_{\text {eff }}=2900 \mathrm{~K}$. The super-solar models, by contrast, possess the strongest Na I lines at warm temperatures, but the NaI lines in these models begin to weaken at $T_{\text {eff }} \leqslant 3600 \mathrm{~K}$, ultimately predicting Na I line strengths that are anti-correlated with metallicity (in the solar/super-solar regime) below $T_{\text {eff }}=3000 \mathrm{~K}$.

The behavior of the Na I doublet in the solar and super-solar synthetic models is not reproduced in our empirical spectra. The metal-rich calibrator, Gl $376 \mathrm{~B}\left([\mathrm{M} / \mathrm{H}]_{\text {SPOCS }}=+0.11\right.$, $[\mathrm{Fe} / \mathrm{H}]_{\text {SPOCS }}=+0.20$ ), is a late-type star (M6) and it exhibits one of the strongest Na I doublets of the 18 calibrators. Since low temperatures favor the formation of molecular species, the anomalous NaI behavior in the synthetic models could be an effect of the treatment of molecular formation at these temperatures. The most recent study of alkali chemistry in cool atmospheres was conducted by Lodders (1999). Lodders (1999) calculated the mole fraction, the amount of an element divided by the total amount of the elements, of neutral $\mathrm{Na}$, ionized $\mathrm{Na}$, and the molecules $\mathrm{NaCl}, \mathrm{NaH}$, and $\mathrm{NaOH}$ as function of 
temperature at 1 bar total pressure for cool dwarf atmospheres $(3300 \mathrm{~K}<T<1500 \mathrm{~K})$. The computations performed by Lodders (1999) indicated that the mole fraction of ionized $\mathrm{Na}$ decreases toward cooler temperatures, while the mole fractions of $\mathrm{NaCl}$ and $\mathrm{NaOH}$ increase; the mole fractions of neutral $\mathrm{Na}$ and $\mathrm{NaH}$ remain almost constant over this temperature range. Indeed, Lodders (1999) found that neutral $\mathrm{Na}$ is the most abundant Na-based species over this temperature range, with the abundance peaking near $\sim 2000 \mathrm{~K}$ and then beginning to decline as $\mathrm{Na}_{2} \mathrm{~S}$ condensation weakens the $\mathrm{Na}$ features in brown dwarf spectra (Lodders 2002). It is possible that these complex chemical networks could play a role in the anomalous behavior of the $\mathrm{Na}$ I feature in the synthetic models: perhaps the most metal-rich models favor the formation of Na-based molecules toward cooler temperatures, for example, such that the neutral $\mathrm{Na}$ responsible for the Na I 2.205 feature depletes more rapidly in metal-rich models, or perhaps $\mathrm{Na}_{2} \mathrm{~S}$ condensation occurs at higher temperatures in the super-solar models. These suggestions are highly speculative, however, and based on the number of odd behaviors exhibited for models cooler than $3000 \mathrm{~K}$, we suspect the root cause is as likely to be computational as astrophysical in nature.

\section{CALIBRATING THE $\mathrm{H}_{2} \mathrm{O}-\mathrm{K} 2$ INDEX AS A SPECTRAL TYPE AND $T_{\text {eff }}$ INDICATOR}

Water is a heteroatomic molecule and the appearance of the absorption band reflects the pressure and temperature sensitivity of the formation of these molecules (Merrill \& Ridgway 1979). Numerous investigators have leveraged this fact to use near-infrared water absorption bands to diagnose $\mathrm{M}$ dwarf effective temperatures and spectral types. Kleinmann \& Hall (1986) noted that the depression at $4778 \mathrm{~cm}^{-1}(2.096 \mu \mathrm{m})$ due to water absorption was largest for dwarf stars, and increases with decreasing spectral type. Jones et al. (1994) placed this calibration on an absolute $T_{\text {eff }}$ scale, deriving effective temperatures for stars with spectral types ranging from M2V to M9V by comparing the observed $\mathrm{H}_{2} \mathrm{O}$ bandhead at $1.34 \mu \mathrm{m}$ to laboratory data for the water absorption coefficient. Ali et al. (1995) similarly created a $T_{\text {eff }}$ indicator using an index sampling the $K$-band $\mathrm{H}_{2} \mathrm{O}$ feature in low resolution spectra at $\sim 2.15$ and $\sim 2.20 \mu \mathrm{m}$, deriving a linear relationship between effective temperature from low-resolution spectra of MK-dwarf standards. More recently, McLean et al. (2003) calculated linear relationships between stellar spectral type and the depth of water absorption bands present in $R \sim 2000$ near-infrared spectra of M, L, and T dwarfs. McLean et al. (2003) found that their $K$-band water index, $\mathrm{H}_{2} \mathrm{O}-\mathrm{D}$, was constant for spectral types M0-M4, at which point the index decreased (due to increased $\mathrm{H}_{2} \mathrm{O}$ absorption) toward cooler spectral types.

To calibrate the $\mathrm{H}_{2} \mathrm{O}-\mathrm{K} 2$ index as a well-defined $T_{\text {eff }}$ indicator, we provide in Table 4 the values of the $\mathrm{H}_{2} \mathrm{O}-\mathrm{K} 2$ indices measured from the BT-Settl-2010 models and originally presented in Figure 4. As Figure 4 demonstrates, the BT-Settl-2010 models predict that the $\mathrm{H}_{2} \mathrm{O}-\mathrm{K} 2$ index is a monotonic function of temperature, but independent of metallicity, for stars in the $3000 \mathrm{~K}<T_{\text {eff }}<4000 \mathrm{~K}$ temperature range. As noted earlier, the lower bound of this metallicity-independent behavior is defined by the clear difference in the behavior of the $\mathrm{H}_{2} \mathrm{O}-\mathrm{K} 2$ index as a function of $T_{\text {eff }}$ for metal-poor and solar/metal-rich stars, respectively. While this low-temperature behavior may be a computational artifact, the metallicity dependent differences at $T_{\text {eff }} \mathrm{s}>4000 \mathrm{~K}$ are much more likely to represent a true astrophysical behaviors. That is, while the $\mathrm{H}_{2} \mathrm{O}$ opacity appears
Table 4

BT-Settl $\mathrm{H}_{2} \mathrm{O}-\mathrm{K} 2$ Indices

\begin{tabular}{|c|c|c|c|c|c|}
\hline \multirow[t]{2}{*}{$T_{\text {eff }}$} & \multicolumn{5}{|c|}{$[\mathrm{M} / \mathrm{H}]$} \\
\hline & -1.0 & -0.5 & 0.0 & +0.3 & +0.5 \\
\hline 4400 & 1.0633894 & 1.0551468 & 1.0459396 & 1.0346350 & \\
\hline 4300 & 1.0610328 & 1.0530623 & 1.0447932 & 1.0325842 & \\
\hline 4200 & 1.0555427 & 1.0486027 & 1.0440688 & 1.0326834 & \\
\hline 4100 & 1.0496983 & 1.0415743 & 1.0399236 & 1.0308537 & \\
\hline 4000 & 1.0419007 & 1.0330761 & 1.0336640 & 1.0265948 & 1.0123681 \\
\hline 3900 & 1.0302266 & 1.0223378 & 1.0250625 & 1.0202581 & 1.0082819 \\
\hline 3800 & 1.0083129 & 1.0047255 & 1.0108148 & 1.0072626 & 0.99948109 \\
\hline 3700 & 0.98369432 & 0.98394169 & 0.98987729 & 0.99077235 & 0.98483467 \\
\hline 3600 & 0.95944974 & 0.95519382 & 0.96753477 & 0.96615374 & 0.96534533 \\
\hline 3500 & 0.93851486 & 0.93301470 & 0.94241768 & 0.93860547 & 0.94239536 \\
\hline 3400 & 0.92003132 & 0.91276339 & 0.91955948 & 0.91273634 & 0.91865987 \\
\hline 3300 & 0.90412690 & 0.89518129 & 0.89743695 & 0.89064011 & 0.89750592 \\
\hline 3200 & 0.88679857 & 0.87540384 & 0.87727299 & 0.86834456 & 0.88154377 \\
\hline 3100 & 0.87051604 & 0.85641549 & 0.85691544 & 0.84896337 & 0.86984104 \\
\hline 3000 & 0.85463719 & 0.83051899 & 0.83022602 & 0.82209357 & 0.86153062 \\
\hline 2900 & 0.84301951 & 0.87462956 & 0.80480058 & 0.79553348 & 0.79291515 \\
\hline 2800 & 0.83379220 & 0.86284561 & 0.77417570 & 0.76562934 & 0.76116386 \\
\hline 2700 & 0.83353582 & 0.85048313 & 0.74699251 & 0.73209011 & 0.72973959 \\
\hline 2600 & 0.83127154 & 0.84092836 & 0.71229056 & 0.69373495 & 0.68974967 \\
\hline 2500 & 0.84359432 & 0.83413574 & 0.68145572 & 0.65795920 & 0.65185812 \\
\hline 2400 & 0.83627202 & 0.82175367 & 0.64586819 & 0.61983636 & 0.61785883 \\
\hline 2300 & 0.84981127 & 0.81318717 & 0.62799732 & 0.60425485 & 0.59720128 \\
\hline 2200 & 0.85792452 & 0.79609664 & 0.58683221 & 0.60165298 & 0.57924869 \\
\hline
\end{tabular}

to "saturate" for even very low metallicities, such that the most metal-poor stars in the model grid do not appear to possess any less $\mathrm{H}_{2} \mathrm{O}$ opacity than the most metal-rich stars of the same temperature, this is not true at the highest temperatures, near the onset of the $\mathrm{H}_{2} \mathrm{O}$ feature in the NIR spectral sequence. Rather, the $\mathrm{H}_{2} \mathrm{O}$ index values tabulated in Table 4 appear to indicate that the $\mathrm{H}_{2} \mathrm{O}$ feature appears at higher temperatures for more metal-rich stars. Care must therefore be exercised in inferring the temperature of stars with very weak $\mathrm{H}_{2} \mathrm{O}$ features; in addition to the greater difficulty of ensuring a high $\mathrm{S} / \mathrm{N}$ measurement of a weak feature, the BT-Settl-2010 models suggest that even if high quality $\mathrm{H}_{2} \mathrm{O}-\mathrm{K} 2$ measurements can be obtained for stars with weak $\mathrm{H}_{2} \mathrm{O}$ absorption, there will be large astrophysical uncertainties introduced into the resultant $T_{\text {eff }}$ estimate due to the strong metallicity dependence of those features at relatively high $T_{\text {eff }} \mathrm{s}$. The gravity dependence of the $\mathrm{H}_{2} \mathrm{O}-\mathrm{K} 2$ index, as a function of model effective temperature and fixed metallicity, is shown in Figure 5. The gravity values of $\log g=4.5$ and 5.0 are the standard values adopted for $\mathrm{M}$ dwarf models. Within the metallicity range explored with the BT-Settl-2010 models, the sensitivity of the $\mathrm{H}_{2} \mathrm{O}-\mathrm{K} 2$ index to surface gravity is negligible for $T_{\text {eff }} \geqslant 3000 \mathrm{~K}$, and throughout the whole effective temperature range studied for the solar and super-solar metallicity models. The largest discrepancies in $\mathrm{H}_{2} \mathrm{O}-\mathrm{K} 2$ index due to surface gravity are found at lower temperatures in the $[\mathrm{M} / \mathrm{H}]=-1.0$ models. Therefore, according to the BT-Settl2010 models, the $\mathrm{H}_{2} \mathrm{O}-\mathrm{K} 2$ index values are metallicity and gravity insensitive for $3000 \mathrm{~K} \leqslant T_{\text {eff }} \leqslant 3800 \mathrm{~K}$.

Effective temperatures for the 133 stars were inferred from their $\mathrm{H}_{2} \mathrm{O}-\mathrm{K} 2$ index using the solar $([\mathrm{M} / \mathrm{H}]=0.0)$ BT-Settl2010 models. For each star, we interpolated the measured $\mathrm{H}_{2} \mathrm{O}-\mathrm{K} 2$ value onto the solar metallicity model $\left[\mathrm{H}_{2} \mathrm{O}-\mathrm{K} 2, T_{\text {eff }}\right]$ grid to get a $T_{\text {eff }}$ estimate and error from its $\mathrm{H}_{2} \mathrm{O}-\mathrm{K} 2$ uncertainty. The values used for the solar metallicity $\left[\mathrm{H}_{2} \mathrm{O}-\mathrm{K} 2, T_{\text {eff }}\right]$ grid are shown in Table 4 . The effective temperatures derived from the 

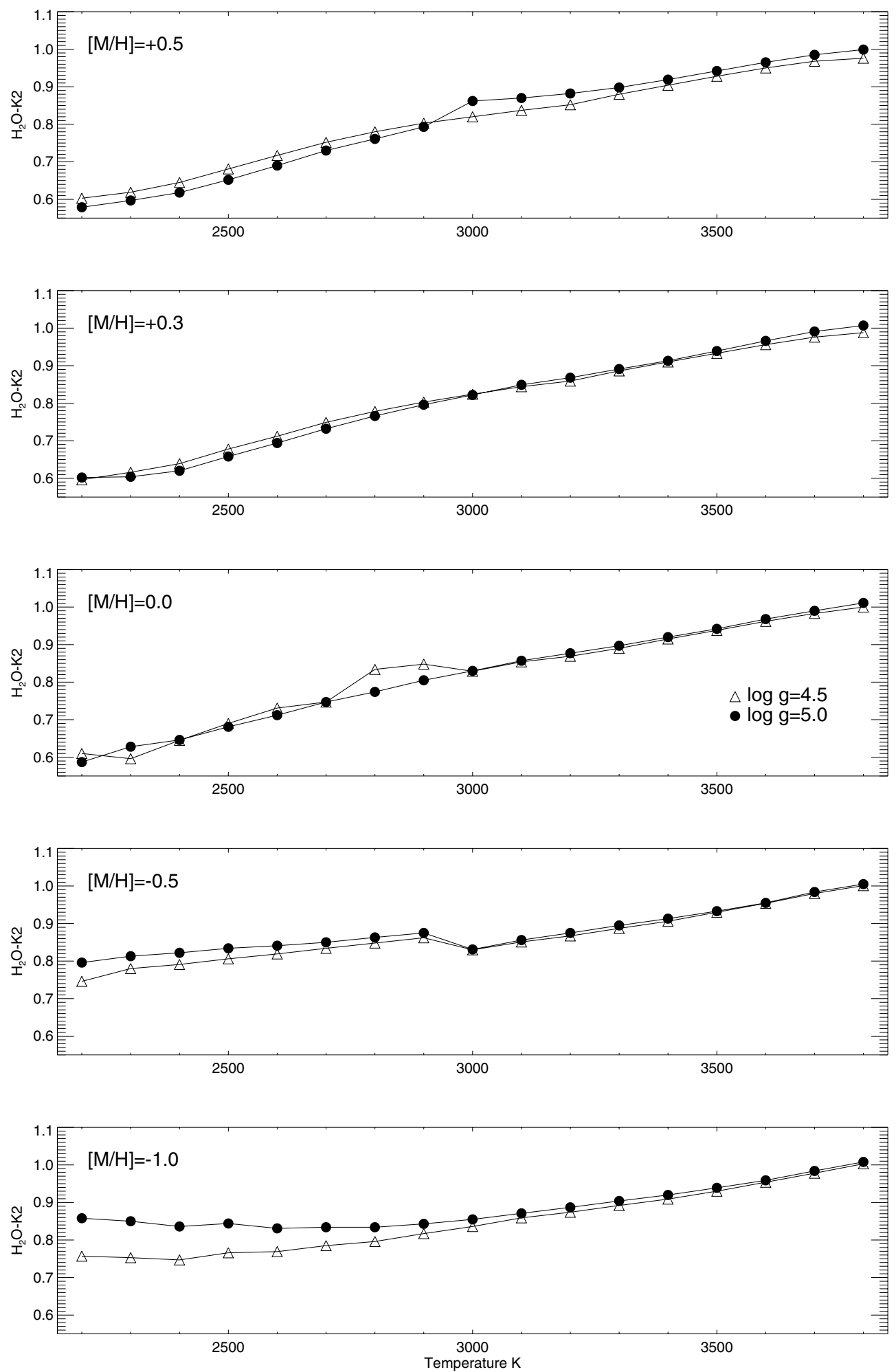

Figure 5. $\mathrm{H}_{2} \mathrm{O}-\mathrm{K} 2$ index measured from the BT-Settl-2010 synthetic spectra shown as a function of model $T_{\text {eff }}$. Triangles represent models with log $g=4.5$ and black dots represent models with $\log g=5.0$. The $\mathrm{H}_{2} \mathrm{O}-\mathrm{K} 2$ index shows negligible sensitivity to surface gravity in all models with $T_{\text {eff }} \geqslant 3000 \mathrm{~K}$. Differences in $\mathrm{H}_{2} \mathrm{O}-\mathrm{K} 2$ index due to surface gravity are small in the solar and super-solar models throughout the whole effective temperature range. The largest discrepancies due to surface gravity are found at lower temperatures in the subsolar $[\mathrm{M} / \mathrm{H}]$ models. The largest discrepancy between the two surface gravities in the $[\mathrm{M} / \mathrm{H}]=0.0 \mathrm{dex}$ models is believed to be a computational artifact.

synthetic spectra should not be considered extremely accurate or reliable, especially for the late-type $\mathrm{M}$ dwarfs in the sample. The BT-Settl-2010 models of $T_{\text {eff }}<3000 \mathrm{~K}$ show $\mathrm{H}_{2} \mathrm{O}$ absorption discrepancies that are more likely to be a computational artifact than an astrophysical difference due to $[\mathrm{M} / \mathrm{H}]$. Due to the finite resolution of the model grid, where models have only been calculated on a grid with a spacing of $\Delta T_{\text {eff }}=100 \mathrm{~K}$, systematic errors of $\sim 100 \mathrm{~K}$ are expected.

The dominant spectral sequence for $\mathbf{M}$ dwarf stars was established two decades ago by Kirkpatrick et al. (1991, hereafter 

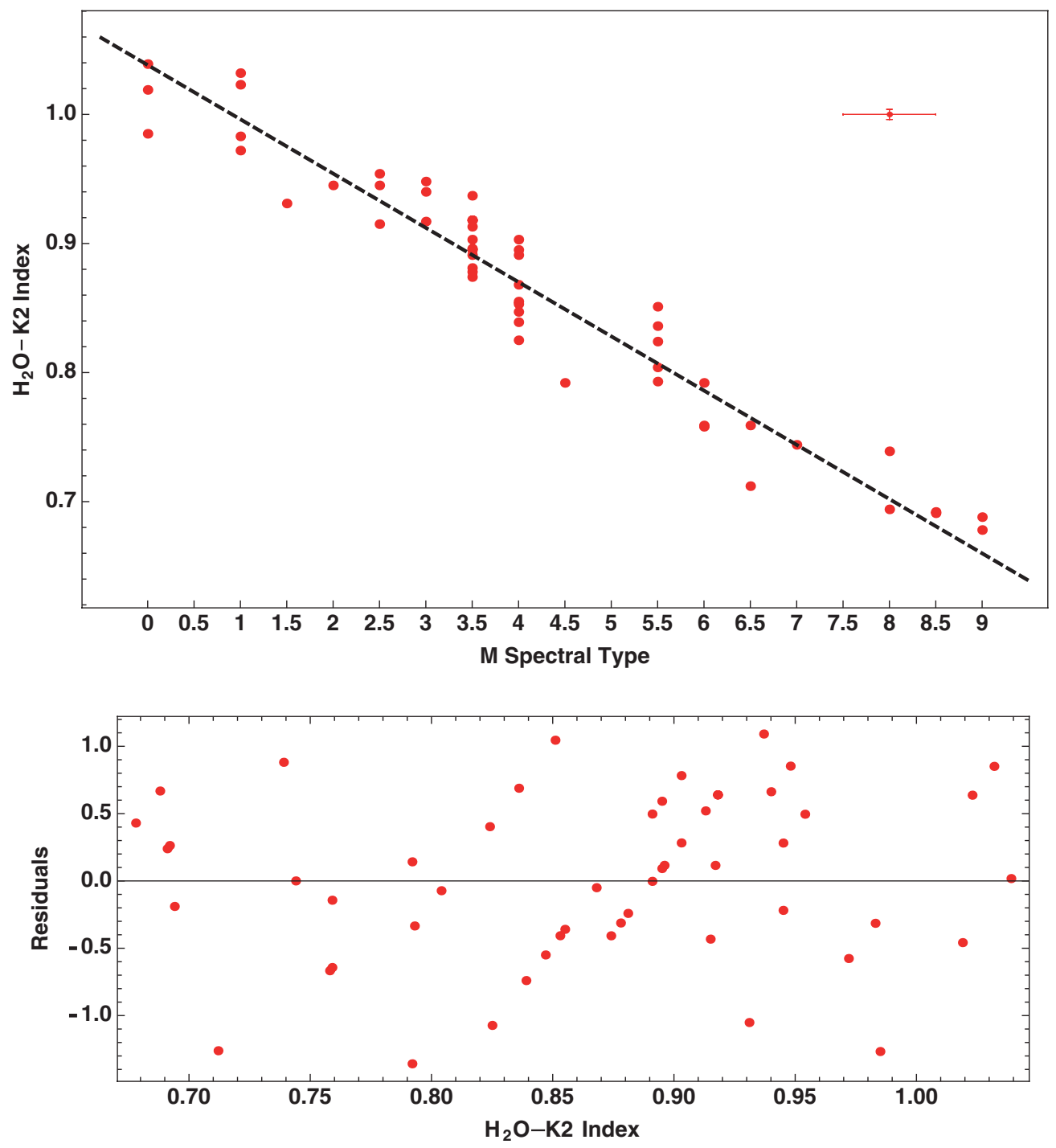

Figure 6. Top: the $\mathrm{H}_{2} \mathrm{O}-\mathrm{K} 2$ index vs. the Spectral Type of M dwarfs. The red dots represent the fifty-four stars with optical KHM spectral types by RECONS. The dashed black line is the linear relationship between KHM spectral types and the $\mathrm{H}_{2} \mathrm{O}-\mathrm{K} 2$ index in Equation (6), obtained using a Bayesian approach that includes the errors in both coordinates of the objects. Bottom: spectral type residuals vs. $\mathrm{H}_{2} \mathrm{O}-\mathrm{K} 2$ index.

(A color version of this figure is available in the online journal.)

KHM), who used red-optical spectra covering 6300-9000 $\AA$ to define a set of primary and secondary $M$ dwarf spectral standards. KHM derived their classifications from a least-squares minimization technique that depends on individual spectral features as well as the overall spectral slope. In the KHM technique, the low resolution spectrum $(18 \AA)$ is only normalized using the almost featureless region near $7500 \AA$ before performing the least-squares minimization, so the overall shape of the spectra is conserved.

Sixty-eight of the stars in our sample have KHM spectral types reported by the Research Consortium On Nearby Stars (RECONS; Henry et al. 2006), enabling us to calibrate the $\mathrm{H}_{2} \mathrm{O}-\mathrm{K} 2$ index as a proxy for KHM spectral type. We exclude from this calibration sample 14 objects with potentially ambiguous $\mathrm{H}_{2} \mathrm{O}-\mathrm{K} 2$ indices: 11 are members of binaries or multiple systems in very tight orbits, such that the final $K$-band spectrum mixes emission from all the stars in the system; we exclude the remaining 3 stars on the basis of their sub-standard telluric/flux calibration. For the 54 single stars in our sample with highquality spectra and KHM spectral types, we performed a linear regression between the measured $\mathrm{H}_{2} \mathrm{O}-\mathrm{K} 2$ indices and $\mathrm{KHM}$ spectral types using a Bayesian approach that takes into account the errors associated with each of the variables (Kelly 2007). According to Kirkpatrick et al. (1991), the KHM spectral types have $\sim 0.5$ subtype errors; the errors associated with each $\mathrm{H}_{2} \mathrm{O}-\mathrm{K} 2$ index are listed in Table 3. This regression analysis indicated that a star's KHM spectral type, expressed as a numerical $\mathrm{M}$ subtype, can be derived from its $\mathrm{H}_{2} \mathrm{O}-\mathrm{K} 2$ indices as

$$
\begin{aligned}
\text { M subype } & =\mathrm{A}+\mathrm{B}\left(\mathrm{H}_{2} \mathrm{O}-\mathrm{K} 2\right) \\
\mathrm{A} & =24.699 \pm 0.930 \\
\mathrm{~B} & =-23.788 \pm 1.067 \\
\mathrm{RMSE}(\text { Msubtype }) & =0.624 .
\end{aligned}
$$

The correlation between KHM spectral type, $\mathrm{H}_{2} \mathrm{O}-\mathrm{K} 2$ index, and the residuals of the fit for our 54 spectral type calibrators is shown in Figure 6. The residuals indicate that the $\mathrm{H}_{2} \mathrm{O}-\mathrm{K} 2$ index can predict each star's spectral type with a typical accuracy of 0.6 sub-types. As Figure 6 shows, however, some stars possess KHM spectral types that differ from the type predicted from their $\mathrm{H}_{2} \mathrm{O}-\mathrm{K} 2$ index by $\gtrsim 1$ subtype. A careful inspection of each of 


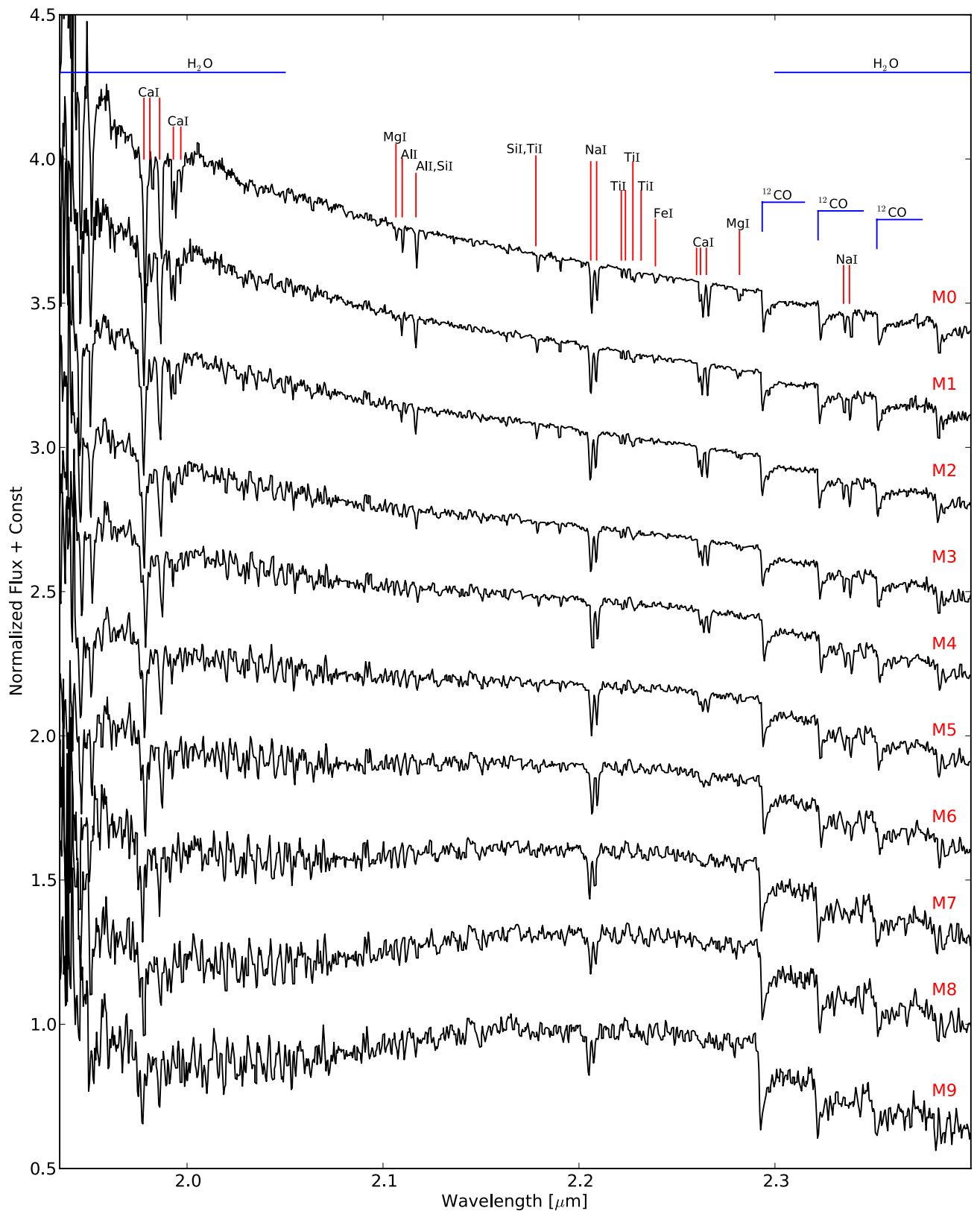

Figure 7. Mean TripleSpec $K$-band spectral type templates. The stars in our sample were organized by spectral type and a template was constructed by combining the spectra in each subtype. All of the subtypes are constituted by at least three stars, and up to as many as thirty, with the exception of the M9 subtype, for which LHS 2924 is the only prototype. The most prominent molecular and atomic features are indicated.

(A color version of this figure is available in the online journal.)

the 54 objects rules out poor quality spectra as the cause of the outliers, but reveals that for the more extreme cases, the overall shape of their $K$-band spectra disagrees with stars of the same KHM spectral type. These discrepancies could be explained by the influence of metallicity on the strengths of double-metal molecular bands, a topic we return to in Section 5.4.

We have used this relationship to infer the KHM spectral type for each star in our sample from its $\mathrm{H}_{2} \mathrm{O}-\mathrm{K} 2$ index; these types are listed in Table 3 and were used to order the sample from earliest to latest spectral types. To provide high quality template spectra for subsequent spectral type measurements, we also combined all the spectra in our sample in bins according to their decimal $\mathrm{H}_{2} \mathrm{O}-\mathrm{K} 2$ types. For each subtype bin, each spectrum was normalized by its mean flux between 2.16 and $2.2 \mu \mathrm{m}$. The spectra then were all cross-correlated with the spectrum of one of the stars in the subtype bin to make their spectral features coincide. Finally, the wavelength offsets were applied to each spectrum. At least 3 stars, and as many as 30, were then median combined to make the master template for each subtype, with the exception of the M9 template, for which LHS 2924 is the only prototype in our sample. The templates from M0 to M9 are shown in Figure 7.

\section{UPDATED AND EXTENDED $K$-BAND METALLICITY CALIBRATIONS}

\subsection{A Revised Empirical [Fe/H] Calibration}

RA10 presented a linear equation for estimating an (early-tomid-) $\mathrm{M}$ star's $[\mathrm{Fe} / \mathrm{H}]$ value based on the strengths of its $\mathrm{Na}$ I and $\mathrm{Ca}$ I lines, and its temperature as diagnosed via its $\mathrm{H}_{2} \mathrm{O}-\mathrm{K}$ 
Table 5

M Dwarf Metallicity Calibration Sample

\begin{tabular}{|c|c|c|c|c|c|}
\hline \multirow[t]{2}{*}{ Name } & \multirow[t]{2}{*}{ Sp. Type } & \multicolumn{2}{|c|}{ SPOCS } & \multicolumn{2}{|c|}{ Predicted } \\
\hline & & {$[\mathrm{M} / \mathrm{H}]$} & {$[\mathrm{Fe} / \mathrm{H}]$} & {$[\mathrm{M} / \mathrm{H}]$} & {$[\mathrm{Fe} / \mathrm{H}]$} \\
\hline Gl 212 & M1 & 0.16 & 0.19 & 0.02 & 0.03 \\
\hline HD $46375 \mathrm{~B}$ & M1 & 0.20 & 0.24 & 0.21 & 0.29 \\
\hline Gl 872 B & M2 & -0.16 & -0.22 & -0.17 & -0.25 \\
\hline G1 797 B & M2 & -0.09 & -0.09 & -0.16 & -0.23 \\
\hline Gl 250 B & M2 & -0.01 & 0.14 & 0.01 & 0.01 \\
\hline NLTT 14186 & M2 & 0.04 & 0.05 & 0.17 & 0.24 \\
\hline HD $222582 \mathrm{~B}$ & M3 & -0.02 & -0.03 & 0.02 & 0.02 \\
\hline Gl 324 B & M4 & 0.25 & 0.31 & 0.22 & 0.31 \\
\hline Gl 768.1 B & M4 & 0.12 & 0.16 & 0.08 & 0.10 \\
\hline Gl 231.1 B & M4 & -0.08 & -0.04 & 0.02 & 0.02 \\
\hline GJ 3348 B & M4 & -0.10 & -0.22 & -0.01 & -0.02 \\
\hline Gl 783.2 B & M4 & -0.09 & -0.15 & -0.20 & -0.29 \\
\hline Gl 544 B & M4 & -0.15 & -0.18 & -0.05 & -0.09 \\
\hline Gl 611 B & M5 & -0.49 & -0.69 & -0.32 & -0.45 \\
\hline Gl 777 B & M5 & 0.19 & 0.21 & 0.03 & 0.03 \\
\hline Gl $166 \mathrm{C}$ & M5 & -0.08 & -0.28 & -0.10 & -0.15 \\
\hline NLTT 15867 & M5 & -0.05 & -0.10 & -0.14 & -0.21 \\
\hline Gl 376 B & M6 & 0.11 & 0.20 & 0.11 & 0.14 \\
\hline
\end{tabular}

index. We present here a revised formalism to describe this relationship, where the revision has been motivated by three factors.

1. The adoption of the revised $\mathrm{H}_{2} \mathrm{O}-\mathrm{K}$ index, as introduced above.

2. The addition of a new M dwarf star, Gl 166C, to the metallicity calibrator sample (Table 5) following the publication of RA10.

3. The adoption of a new functional form to estimate the star's metallicity from its $K$-band spectral features.

The adoption of a new functional form for the $K$-band metallicity calibration is perhaps the most meaningful change and deserves further explanation. The linear expression in RA10 produced unrealistically high $[\mathrm{Fe} / \mathrm{H}]$ values for some of the more metal-rich $\mathrm{M}$ dwarfs in this sample (e.g., LHS 3799; $[\mathrm{Fe} / \mathrm{H}] \sim+0.64$ according to the RA10 calibration). While it is necessary to include the water index in the relationship to remove the temperature dependence of the $\mathrm{Na}$ I and $\mathrm{Ca}$ I features, the assumption that water absorption should be included as an independent variable is not necessarily correct. Extensive experimentation with various functional forms revealed that a better $[\mathrm{Fe} / \mathrm{H}]$ calibration fit could be obtained if water absorption is used as a "weight" for the $\mathrm{Na}$ I and Ca I strengths rather than as a fully independent variable.

We conducted multivariate linear regressions on the $\mathrm{Na}$ I and Ca I EWs, weighted by each star's $\mathrm{H}_{2} \mathrm{O}-\mathrm{K} 2$ index, for the 18 metallicity calibrators to identify the best-fit relationship to predict each star's $[\mathrm{Fe} / \mathrm{H}]$ value. The resulting calibration is

$$
\begin{aligned}
{[\mathrm{Fe} / \mathrm{H}] } & =\mathrm{A}+\mathrm{B} \frac{\mathrm{NaI}_{\mathrm{EW}}}{\mathrm{H}_{2} \mathrm{O}-\mathrm{K} 2}+\mathrm{C} \frac{\mathrm{CaI}_{\mathrm{EW}}}{\mathrm{H}_{2} \mathrm{O}-\mathrm{K} 2} \\
\mathrm{~A} & =-1.039 \pm 0.170 \\
\mathrm{~B} & =0.092 \pm 0.023 \\
\mathrm{C} & =0.119 \pm 0.033 \\
\operatorname{RMSE}([\mathrm{Fe} / \mathrm{H}]) & =0.141 .
\end{aligned}
$$

For completeness, we summarize in Appendix A the residual mean square (RMSp), the root-mean-squared error (RMSE), and adjusted square for the multiple correlation coefficient $\left(R_{\mathrm{ap}}^{2}\right)$ statistics for evaluating the uncertainties and predictive power associated with a functional fit to a given set of calibration data. These quantities were introduced by Schlaufman \& Laughlin (2010) to compare the quality of the photometric $[\mathrm{Fe} / \mathrm{H}]$ calibrations. The $[\mathrm{Fe} / \mathrm{H}]$ fit in $\mathrm{RA} 10$ has an $\mathrm{RMSp}([\mathrm{Fe} / \mathrm{H}])=$ 0.02 and an $R_{\mathrm{ap}}^{2}([\mathrm{Fe} / \mathrm{H}])=0.63$. The addition of another binary system and the new independent variables maintained or improved each of these statistics with respect to the RA10 calibration: the RA10 $[\mathrm{Fe} / \mathrm{H}]$ calibration and the revision presented here possess equivalent RMSp values, but the new $[\mathrm{Fe} / \mathrm{H}]$ fit has a higher $R_{\mathrm{ap}}^{2}\left(R_{\mathrm{ap}}^{2}\left([\mathrm{Fe} / \mathrm{H}]_{\mathrm{RA} 11}\right)=0.67\right)$.

For the $[\mathrm{Fe} / \mathrm{H}]$ model in Equation (7), the root-mean-squared error of validation $\left(\mathrm{RMSE}_{V}\right.$, Appendix A) is equal to 0.161 dex. The $\mathrm{RMSE}_{V}$ value is equivalent to a $\sim 70 \%$ confidence interval or $1 \sigma$ and is considered a sensible estimate of average prediction error. The $95 \%$ confidence interval is equal to \pm 0.344 dex.

The $[\mathrm{Fe} / \mathrm{H}]$ residuals versus the dependent variables of the fit in Equation (7) and the SPOCS $[\mathrm{Fe} / \mathrm{H}]$ values are shown in Figure 8. The residuals show mostly random scatter, and no evident structure can be seen as a function of the weighted line strengths (i.e., left and middle panels), and the computed $[\mathrm{Fe} / \mathrm{H}]$ from Equation (7) (right panel). The "outlier" in the bottom left of the $[\mathrm{Fe} / \mathrm{H}]$ residuals versus the SPOCS $[\mathrm{Fe} / \mathrm{H}]$ values plot is the lowest metallicity star in the calibration sample, Gl $611 \mathrm{~B}$, however, and demonstrates the pressing need for an expanded sample of metallicity calibrators to establish if any systematic errors are present, and if so, remove them.

The EWs of the CaI triplet and the NaI doublet features, weighted by the $\mathrm{H}_{2} \mathrm{O}-\mathrm{K} 2$ index, are plotted in Figure 9 for all the $\mathrm{M}$ dwarfs analyzed here. Isometallicity $[\mathrm{Fe} / \mathrm{H}]$ contours calculated from Equation (7) are shown as dashed and dotted lines. In this plane, there is a clear distinction between the $M$ dwarfs in the calibration sample with metal-rich and metalpoor FGK-dwarf companions. The M dwarf planet hosts also all have $[\mathrm{Fe} / \mathrm{H}]>0.0$ dex according to this relationship, with the exception of Gl 581 and Gl 649, whose $[\mathrm{Fe} / \mathrm{H}]$ are equal to -0.10 dex and -0.04 dex, respectively. The $[\mathrm{Fe} / \mathrm{H}]$ values predicted by Equation (7) for the full sample of stars analyzed here are listed in Table 3: the most iron-rich star in the sample is the planet host HIP 79431(+0.46 dex) and the least iron-rich is the flare star $\mathrm{V}^{*} \mathrm{~V} 1513 \mathrm{Cyg}(-0.64 \mathrm{dex})$.

\subsection{A New K-band Overall Metallicity $([M / H])$ Calibration}

In addition to iron abundance estimates, the SPOCS catalog also provides overall metallicity $[\mathrm{M} / \mathrm{H}]$ values for the FGKcompanions of stars in the calibration sample. In the fitting procedure described in Valenti \& Fischer (2005), the $[\mathrm{M} / \mathrm{H}]$ value is an individual model parameter, rather than a quantity constructed from individual element abundances. We performed a linear regression as described above, assuming the same functional form as adopted for the iron metallicity estimate, to calibrate the relationship between the water weighted $\mathrm{Na}$ I doublet and $\mathrm{Ca}$ I triplet and $[\mathrm{M} / \mathrm{H}]$ :

$$
\begin{aligned}
{[\mathrm{M} / \mathrm{H}] } & =\mathrm{A}+\mathrm{B} \frac{\mathrm{NaI}_{\mathrm{EW}}}{\mathrm{H}_{2} \mathrm{O}-\mathrm{K} 2}+\mathrm{C} \frac{\mathrm{CaI}_{\mathrm{EW}}}{\mathrm{H}_{2} \mathrm{O}-\mathrm{K} 2} \\
\mathrm{~A} & =-0.731 \pm 0.120 \\
\mathrm{~B} & =0.066 \pm 0.016 \\
\mathrm{C} & =0.083 \pm 0.023 \\
\operatorname{RMSE}([\mathrm{M} / \mathrm{H}]) & =0.100 .
\end{aligned}
$$



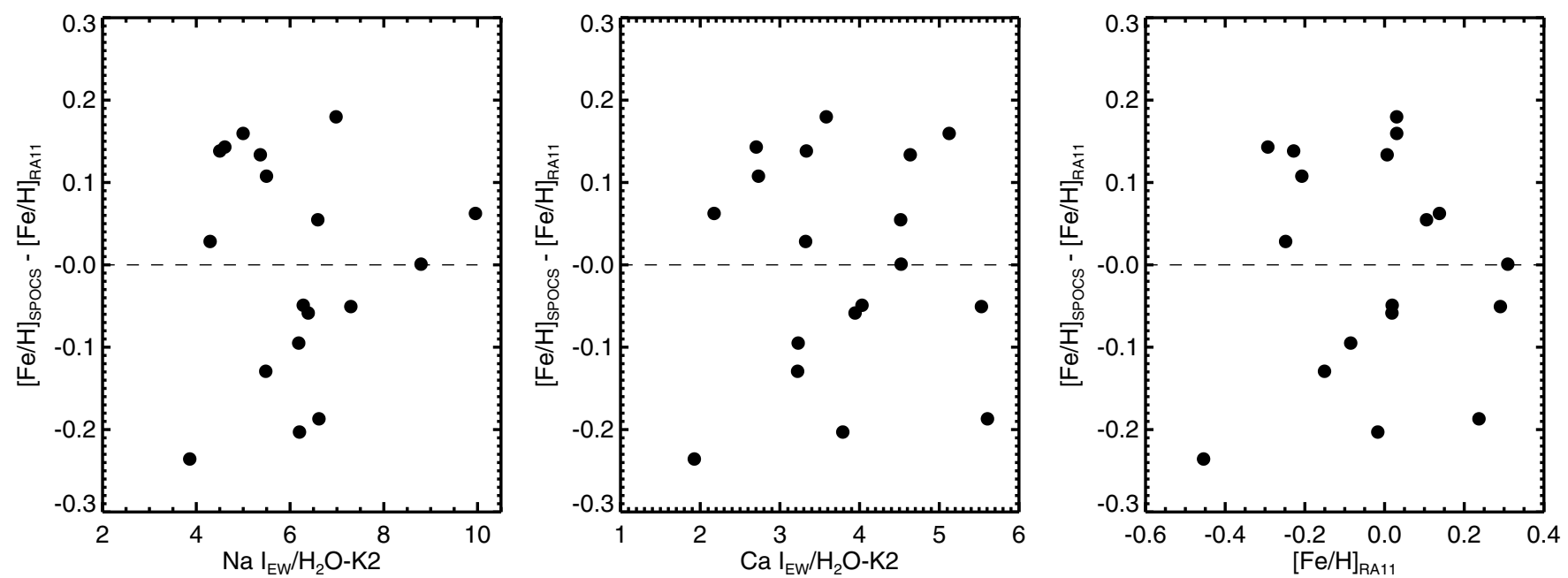

Figure 8. $[\mathrm{Fe} / \mathrm{H}]$ residuals vs. the independent variables of the $[\mathrm{Fe} / \mathrm{H}]$ fit in Equation (7) $\left(\mathrm{Na} / / \mathrm{H}_{2} \mathrm{O}-\mathrm{K} 2\right.$, left; $\mathrm{Ca} \mathrm{I} / \mathrm{H}_{2} \mathrm{O}-\mathrm{K} 2$, middle), and as a function of the predicted metallicity (right).

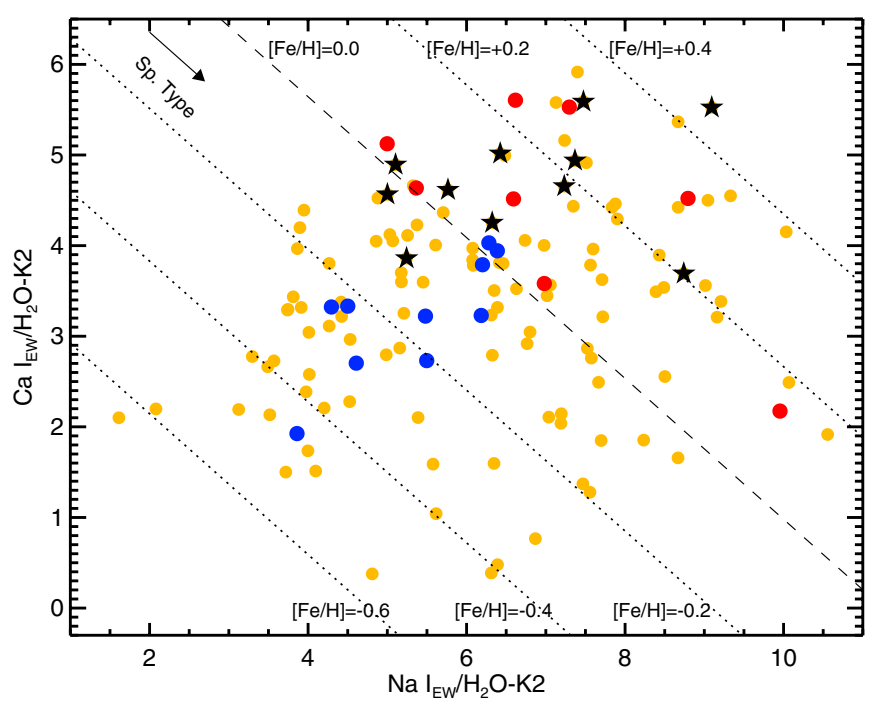

Figure 9. EW of the CaI vs. the EW of the NaI, both weighted by the $\mathrm{H}_{2} \mathrm{O}-\mathrm{K} 2$ index, for the $\mathrm{M}$ dwarfs in our sample. The red and blue dots are the $18 \mathrm{M}$ dwarfs in the metallicity calibration sample with $[\mathrm{Fe} / \mathrm{H}] \geqslant 0.0$ and $[\mathrm{Fe} / \mathrm{H}]<0.0$, respectively. The black stars represent the $\mathrm{M}$ dwarf planet hosts, and the yellow dots the rest of the $\mathrm{M}$ dwarfs studied in this work. The dashed line is an isometallicity contour for $[\mathrm{Fe} / \mathrm{H}]=0.0$. The dotted lines are isometallicity contours for $[\mathrm{Fe} / \mathrm{H}]$ values $+0.4,+0.2,-0.2,-0.4$, and -0.6 dex from the top right corner to the bottom left corner, respectively. The isometallicity contours were calculated from Equation (7). In this plane, there is a clear distinction between the $\mathrm{M}$ dwarfs in the calibration sample with metal-rich and metalpoor FGK-dwarf companions. The $\mathrm{M}$ dwarf planet hosts all have $[\mathrm{Fe} / \mathrm{H}]>$ 0.0 dex, except Gl 581 and Gl 649, whose $[\mathrm{Fe} / \mathrm{H}]$ are equal to -0.10 dex and -0.04 dex, respectively. The most iron-rich star in the sample is the planet host HIP 79431(+0.46 dex) and the least iron-rich is the flare star V* V1513 Cyg $(-0.64$ dex $)$.

(A color version of this figure is available in the online journal.)

The $K$-band $[\mathrm{M} / \mathrm{H}]$ fit has an $\mathrm{RMSp}([\mathrm{M} / \mathrm{H}])=0.010$ and an adjusted $R$ square $R_{\text {ap }}^{2}([\mathrm{M} / \mathrm{H}])=0.67$. For the $[\mathrm{M} / \mathrm{H}]$ model in Equation (8), the $\mathrm{RMSE}_{V}$ is equal to $0.111 \mathrm{dex}(\sim 70 \%$ confidence interval or $1 \sigma$ ) and the $95 \%$ confidence interval is equal to \pm 0.237 . None of the previous photometric or spectroscopic works on M dwarf metallicities have estimated $[\mathrm{M} / \mathrm{H}]$ values, therefore, a comparison to other methods cannot be performed. The $[\mathrm{M} / \mathrm{H}]$ residuals versus the dependent variables of the fit in Equation (8) and the SPOCS $[\mathrm{M} / \mathrm{H}]$ values are shown in Figure 10. The residuals show just random scatter; as with the $[\mathrm{Fe} / \mathrm{H}]$ residuals, no structure is evident in the residuals as function of the normalized $\mathrm{Na}$ and $\mathrm{Ca}$ line strengths or the computed $[\mathrm{M} / \mathrm{H}]$ values.

Figure 11 shows the same $\mathrm{Ca} / \mathrm{H}_{2} \mathrm{O}-\mathrm{K} 2$ versus $\mathrm{Na} / \mathrm{H}_{2} \mathrm{O}-\mathrm{K} 2$ plane as Figure 9, but the dashed and dotted lines represent iso-overall-metallicity contours calculated from Equation (8). The $\mathrm{M}$ dwarf planet hosts all have $[\mathrm{M} / \mathrm{H}]>0.0$ dex, with the exception of Gl 581 and Gl 649 (the same stars that were subsolar in $[\mathrm{Fe} / \mathrm{H}]$ ) with $[\mathrm{M} / \mathrm{H}]$ values equal to -0.06 dex and -0.02 dex, respectively. The most metal-rich star in the sample HIP 79431 has a $[\mathrm{M} / \mathrm{H}]=+0.33$ dex and the least metal-rich $\mathrm{V}^{*}$ V1513 Cyg has $[\mathrm{M} / \mathrm{H}]=-0.45 \operatorname{dex}$. The predicted $[\mathrm{M} / \mathrm{H}]$ values from Equation (8) for the stars in our sample are shown in Table 3.

As the BT-Settl-2010 theoretical model atmospheres are calibrated as a function of their overall metallicity, we are now able to directly compare the feature strengths measured from our model grid against the isometallicity contours we inferred from the empirical calibration sample above. Figure 12 shows the EW of Ca I versus the EW of Na I, both weighted by $\mathrm{H}_{2} \mathrm{O}-\mathrm{K} 2$ that we measured from the BT-Settl-2010 model spectra with temperatures equal to or higher than $3000 \mathrm{~K}$. This plane splits the synthetic models by metallicity, in a similar way that it also splits the calibration stars for the metallicity fits. The $[\mathrm{M} / \mathrm{H}]=-1.0$ dex models lie in the bottom-left corner, and the $[\mathrm{M} / \mathrm{H}]=+0.5$ dex models lie upward and to the right of the $[\mathrm{M} / \mathrm{H}]=-1.0$ dex models, consistent with the direction of the offsets predicted by the isometallicity contours. However, since the synthetic models predict weaker Na I doublet and $\mathrm{Ca}$ I triplet features than are observed in the empirical spectra, the models do not directly align with the empirical isometallicity contours (depicted by dashed lines) obtained with the $K$-band $[\mathrm{M} / \mathrm{H}]$ fit of Equation (8). The $[\mathrm{M} / \mathrm{H}]=-1.0$ dex models are located above the $[\mathrm{M} / \mathrm{H}]=-0.6$ dex empirical contour, while the $[\mathrm{M} / \mathrm{H}]=+0.5$ dex model points are far below the $[\mathrm{M} / \mathrm{H}]=$ 0.0 dex empirical contour. The odd behavior of the Na I doublet features of the super-solar models mentioned above becomes evident as temperature decreases in Figure 12. Subsolar and solar models of temperatures between $3700 \mathrm{~K}$ and $3000 \mathrm{~K}$ appear as parallel lines in this plane, but the super-solar models intersect the solar model at $3000 \mathrm{~K}$. 

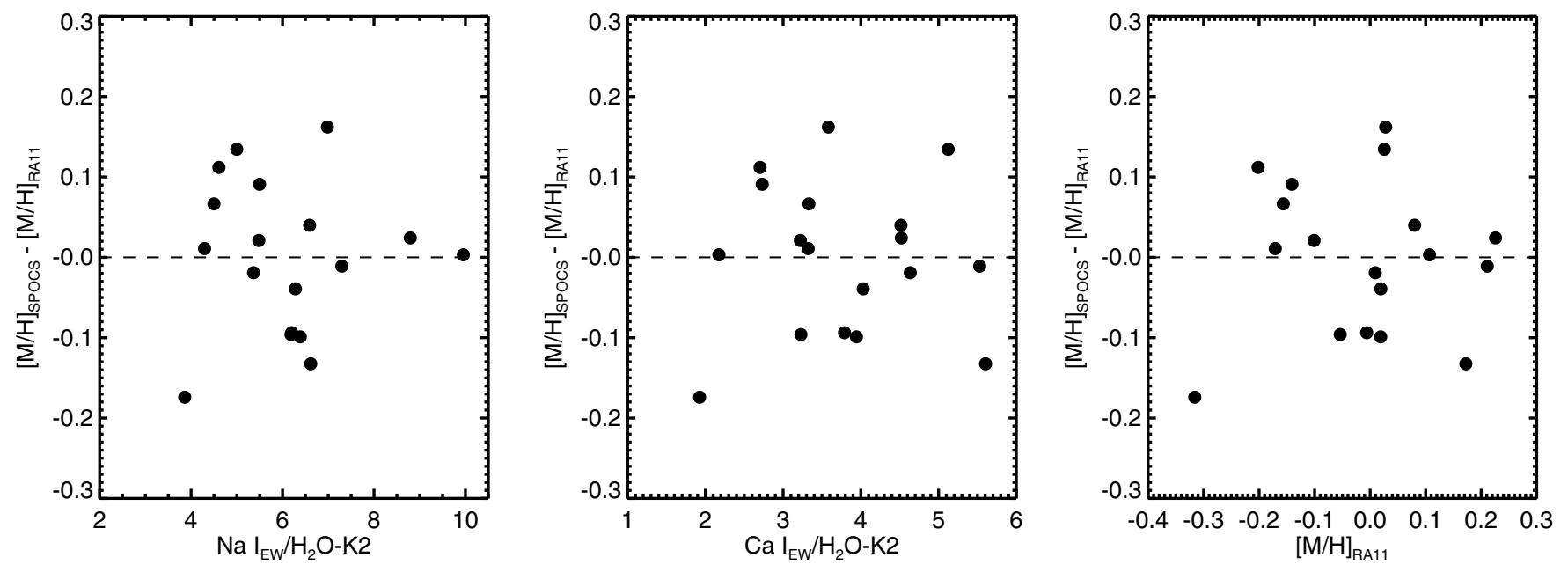

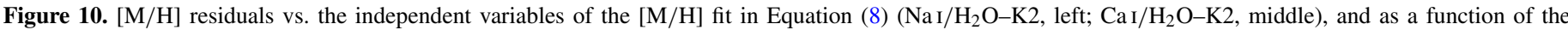
predicted metallicity (right)

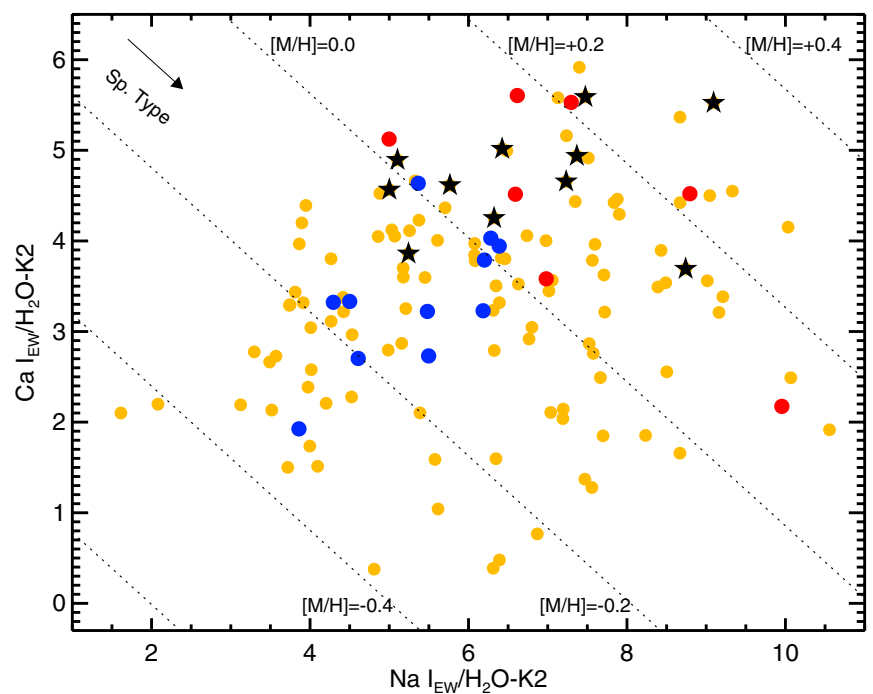

Figure 11. EW of the Ca I vs. the EW of the $\mathrm{Na}$ I both weighted by the $\mathrm{H}_{2} \mathrm{O}-\mathrm{K} 2$ index, for the M dwarfs in our sample. The nomenclature is the same as Figure 9. The dashed line is an isometallicity contour for $[\mathrm{M} / \mathrm{H}]=0.0$. The dotted lines are isometallicity contours for $[\mathrm{M} / \mathrm{H}]$ values $+0.4,+0.2,-0.2$, and -0.4 dex from the top right corner to the bottom left corner, respectively. The isometallicity contours were calculated from Equation (8). The $\mathrm{M}$ dwarf planet hosts all have $[\mathrm{M} / \mathrm{H}]>0.0$ dex, except $\mathrm{Gl} 581$ and $\mathrm{Gl649}$, whose $[\mathrm{M} / \mathrm{H}]$ are equal to -0.06 dex and -0.02 dex. The most metal-rich star in the sample HIP 79431 has an $[\mathrm{M} / \mathrm{H}]=+0.33$ dex and the least metal-rich $\mathrm{V}^{*} \mathrm{~V} 1513 \mathrm{Cyg}$ has $[\mathrm{M} / \mathrm{H}]=-0.45$ dex.

(A color version of this figure is available in the online journal.)

\subsection{The $\mathrm{K}$-band $[\mathrm{Fe} / \mathrm{H}]$ Method Compared with Other $[\mathrm{Fe} / \mathrm{H}]$ Techniques}

\subsubsection{Statistics of the $\mathrm{K}$-band $[\mathrm{Fe} / \mathrm{H}]$ and Photometric Methods}

We now seek to compare the quality of our $[\mathrm{Fe} / \mathrm{H}] \mathrm{fit}$, and the resultant values it predicts for our calibration stars, to similar evaluations of the $[\mathrm{Fe} / \mathrm{H}]$ predictions produced by the photometric calibrations. Neves et al. (2012, hereafter N12) performed a similar exercise, comparing the RMSp, $R_{\mathrm{ap}}^{2}$, and the dispersion around the mean (rms) values of the photometric metallicity scales using a sample of $23 \mathrm{M}$ dwarfs with solar-type companions of known metallicity, and refined the Schlaufman \& Laughlin (2010) calibration based on their results.

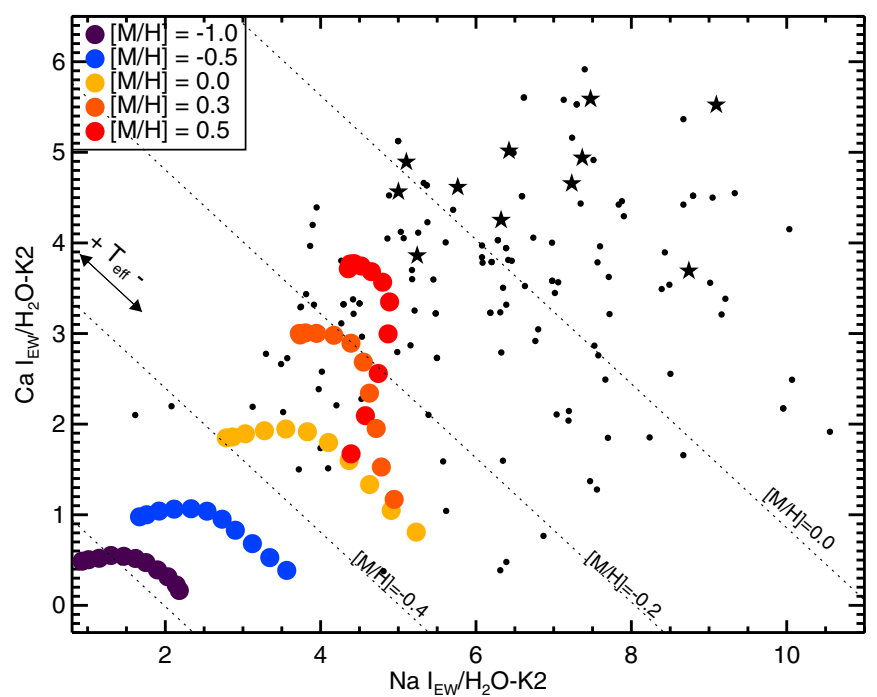

Figure 12. EW of CaI vs. EW of $\mathrm{Na}$, weighted by $\mathrm{H}_{2} \mathrm{O}-\mathrm{K} 2$ index, for the BT-Settl-2010 synthetic models by Allard et al. (2010) with $T_{\text {eff }} \leqslant 3000 \mathrm{~K}$. The dashed lines are isometallicity contours for $[\mathrm{M} / \mathrm{H}]$ values, from the top right corner to the bottom left corner, of $-0.2,-0.4$, and -0.6 . The isometallicity contours for $[\mathrm{M} / \mathrm{H}]$ were calculated from Equation (8). There is a clear distinction between the different metallicity models in this plane. The BT-Settl2010 synthetic models follow the same direction as the isometallicity contours but their weaker strengths of the $\mathrm{Na}$ I doublet and $\mathrm{Ca}$ I triplet do not allow a direct metallicity comparison with empirical data. Subsolar and solar models of temperatures between $3700 \mathrm{~K}$ and $3000 \mathrm{~K}$ appear as parallel lines in this plane, but the super-solar models intersect the solar model at $3000 \mathrm{~K}$, due to the odd behavior of the $\mathrm{Na}$ I doublet features of the super-solar models mentioned in the text.

(A color version of this figure is available in the online journal.)

Unfortunately, for the same reason that N12 did not include the RA10 metallicity predictions in their analysis, because our calibrator samples do not overlap well, it is difficult to directly compare the statistics of our fit to those computed by N12. N12 analyzed $23 \mathrm{M}$ dwarfs with reliable metallicity estimates, $V$ magnitudes, and trigonometric parallaxes; of these, only seven are members of our sample of $\mathbf{M}$ dwarfs with moderate resolution NIR spectra, providing minimal leverage for evaluating the accuracy of the various techniques with a common set of calibrators. This demonstrates an important point: the statistics calculated above, by definition, describe 
Table 6

Statistics of the $[\mathrm{Fe} / \mathrm{H}]$ Prediction Models

\begin{tabular}{llrcccr}
\hline \hline Calibration & $p$ & $N$ & rms & RMSp & $R_{\text {ap }}^{2}$ & Source \\
\hline B05 & 5 & 48 & $0.483 \pm 0.047$ & $0.037 \pm 0.006$ & $0.858 \pm 0.028$ & Table $^{\text {a }}$ \\
JA09 & 1 & 6 & $0.082 \pm 0.016$ & $0.004 \pm 0.002$ & $0.472 \pm 1.224$ & Table $1^{\text {b }}$ \\
SL10 & 1 & 19 & $0.144 \pm 0.017$ & $0.022 \pm 0.005$ & $0.489 \pm 0.188$ & Table $1^{\text {c }}$ \\
N12 & 2 & 23 & $0.167 \pm 0.035$ & $0.034 \pm 0.010$ & $0.426 \pm 0.238$ & Table 2 ${ }^{\text {d }}$ \\
RA11 & 3 & 18 & $0.208 \pm 0.030$ & $0.020 \pm 0.004$ & $0.670 \pm 0.161$ & Table 5
\end{tabular}

Notes. (a) Bonfils et al. 2005a; (b) Johnson \& Apps 2009; (c) Schlaufman \& Laughlin 2010; (d) N12.

how well a given formalism can reproduce measurements of a particular calibration set. The meaning of those statistics, therefore, is inextricably linked to the particular calibration set from which they were computed: comparing those statistics among various metallicity calibrations requires that the statistics be computed with respect to a specific, and shared, set of calibrators.

To make matters worse, the veracity of the calibration set is as important, if not more so, as the number of calibrators. For example, while the B05 functional fit has overall better statistics (as we show below), some of the stars in its calibration sample have poor photometry values. The JA09, SL10, and N12 calibrations improved the B05 calibration by including only stars with high precision photometry/parallax measurements, excluding several of the stars in B05, and including stellar population and kinematic considerations. Moreover, we find differences up to 0.3 dex in the $[\mathrm{Fe} / \mathrm{H}]$ values assigned for the $\mathrm{M}$ dwarfs in our calibration sample and the values used to derived the photometric scales. For example, B05, SL10, and N12 assign an $[\mathrm{Fe} / \mathrm{H}]$ value of -0.15 dex to Gl $250 \mathrm{~B}$, while the SPOCS $[\mathrm{Fe} / \mathrm{H}]$ value we adopt for this star in our own calibration sample is +0.14 dex. ${ }^{12}$ As the metallicity calibrations were derived with reference to these $[\mathrm{Fe} / \mathrm{H}]$ values, the choice of which set of $[\mathrm{Fe} / \mathrm{H}]$ values to adopt as a benchmark will necessarily bias the comparison toward the calibrations derived from those $[\mathrm{Fe} / \mathrm{H}]$ values. As a result, a truly fair comparison of the photometric and spectroscopic techniques will only be possible once the calibration sample has been significantly enlarged, and the veracity of the $[\mathrm{Fe} / \mathrm{H}]$ values adopted for the calibration sample has been well established.

Given the limitations outlined above, we conclude that the fairest comparison of the spectroscopic and photometric techniques that can currently be made is to qualitatively compare the internal quality of each fit, as benchmarked by its native calibration sample, as well as to compare the size and character of the calibration sample from which each fit was derived. Table 6 lists the rms, RMSp, and $R_{\text {ap }}^{2}$ values for each calibration technique, along with the number of predictors $p$ and sample size $N$ for each model. The uncertainties were estimated using the bootstrapping method described in N12.

The number of calibrators and the dispersion around the mean value evaluate the quality of the calibration sample that each technique is based on. Large samples (large $N$ ) that cover a large range of values (large dispersion) are preferred. According to Table 6 , the B50 calibration sample is the largest $(N=48)$, and also the most diverse $(r m s=0.483)$, followed by our calibration (RA11), SL10, and N12, respectively. The JA09 calibration

\footnotetext{
12 We note that as this paper went to press, Önehag et al. (2011) reported a third metallicity for G1 250B of -0.05 dex, based on an analysis of

high-resolution ( $R \sim 50,000)$ high signal-to-noise $J$-band spectrum of Gl 250 $\mathrm{B}$ itself.
}

sample has a very low rms value since it is composed only of high $[\mathrm{Fe} / \mathrm{H}]$ stars.

The RMSp and $R_{\text {ap }}^{2}$ statistics evaluate the internal quality of each functional fit; they describe how well the values predicted by each fit match the measured for the calibrator stars that were used to construct the fit. Small RMSp values (small residuals) and $R_{\text {ap }}^{2}$ values closer to 1 are preferred (see Appendix A). The JA09 fit has the smallest RMSp, but its $R_{\text {ap }}^{2}$ has a large uncertainty. N12 noted that the $R_{\mathrm{ap}}^{2}$ is a noisy statistical estimator for small samples, however, and the JA09 fit only has six stars in its calibration sample. The B05 fit has the largest $R_{\text {ap }}^{2}$.

In summary, the B05 calibration has the better statistics when its native calibration sample is used. However, the B05 calibration underestimates $\mathrm{M}$ dwarf metallicities, probably a result of using old visual magnitudes, instead of Johnson $V$ band magnitudes, and the lack of high $[\mathrm{Fe} / \mathrm{H}] \mathrm{M}$ dwarfs in their calibration sample. The JA09 fit has a very small calibration sample composed only of high metallicity $\mathrm{M}$ dwarfs, and therefore, not a reliable fit for $[\mathrm{Fe} / \mathrm{H}]$ prediction for $\mathrm{M}$ dwarfs. We find that the RA11, SL10, and N12 fits have calibration samples of similar size, and similar rms and RMSp values; our calibration has the highest $R_{\mathrm{ap}}^{2}$ values of the three. However, as noted earlier, none of these statistics can evaluate the veracity of the input values of the calibration sample, which determines the accuracy of the predicted values by the functional fit, as the recalibration of the $\mathrm{B} 05$ fit demonstrates. Constructing an enlarged calibration sample and demonstrating the veracity of the adopted $[\mathrm{Fe} / \mathrm{H}]$ estimates are key steps to enable a full and detailed comparison of the reliability of these techniques.

\subsection{2. $\mathrm{K}$-band $[\mathrm{Fe} / \mathrm{H}]$ Estimates Compared with Other $[\mathrm{Fe} / \mathrm{H}]$ Techniques}

Super-solar metallicities for most of the M dwarf planet hosts are predicted by the $K$-band $[\mathrm{Fe} / \mathrm{H}]$ fit as well as the photometric calibrations by Johnson \& Apps (2009) and Schlaufman \& Laughlin (2010). While these three methods agree on the systematic metal-richness of planet hosts, the $[\mathrm{Fe} / \mathrm{H}]$ values they predict do differ for each of the planet hosts (Table 9).

Photometric $[\mathrm{Fe} / \mathrm{H}]$ methods need accurate $V$ magnitudes and distances, to calculate absolute $K_{S}$ magnitudes, and thereby infer the metallicities of M dwarfs from the $M_{V}$ versus $V-K_{S}$ color-magnitude diagram. Forty-eight of the $\mathrm{M}$ dwarfs in our sample have accurate Johnson- $V$ magnitudes and parallaxes by HIPPARCOS (van Leeuwen 2007), and $K_{S}$ magnitudes by 2MASS (Skrutskie et al. 2006). By including the M dwarfs with Johnson $V$ magnitudes and parallaxes in the Yale Trigonometric Parallaxes Catalogue (van Altena et al. 2001), we can assemble a sample of ninety stars with the necessary data to compare the results from the new $K$-band $[\mathrm{Fe} / \mathrm{H}]$ fit and photometric $[\mathrm{Fe} / \mathrm{H}]$ relations. 
Table 7

$[\mathrm{Fe} / \mathrm{H}]$ Comparison Sample

\begin{tabular}{|c|c|c|c|c|c|c|}
\hline Name & $\begin{array}{c}\mathrm{B} 05 \\
{[\mathrm{Fe} / \mathrm{H}]}\end{array}$ & $\begin{array}{c}\text { JA09 } \\
{[\mathrm{Fe} / \mathrm{H}]}\end{array}$ & $\begin{array}{c}\text { SL10 } \\
{[\mathrm{Fe} / \mathrm{H}]}\end{array}$ & $\begin{array}{c}\text { W09 } \\
{[\mathrm{Fe} / \mathrm{H}]}\end{array}$ & $\begin{array}{c}\text { RA11 } \\
{[\mathrm{Fe} / \mathrm{H}]}\end{array}$ & $\begin{array}{c}\text { L07 } \\
\text { Class }\end{array}$ \\
\hline Gl 699 & $\cdots$ & $\ldots$ & -0.76 & $\cdots$ & -0.39 & $\mathrm{dM}$ \\
\hline Gl 411 & -0.34 & $\ldots$ & $\ldots$ & -0.14 & -0.41 & $\mathrm{dM}$ \\
\hline G1 905 & $\ldots$ & 0.17 & 0.14 & $\ldots$ & 0.19 & $\mathrm{dM}$ \\
\hline Gl 447 & $\ldots$ & $\ldots$ & -0.28 & $\ldots$ & -0.01 & $\mathrm{dM}$ \\
\hline Gl 725 B & -0.37 & $\ldots$ & -0.44 & -0.04 & -0.36 & $\mathrm{dM}$ \\
\hline Gl 725 A & -0.32 & $\ldots$ & -0.29 & 0.01 & -0.49 & $\mathrm{dM}$ \\
\hline GJ 1111 & $\ldots$ & $\ldots$ & 0.25 & $\ldots$ & -0.19 & $\mathrm{dM}$ \\
\hline Gl 273 & -0.15 & 0.07 & -0.06 & 0.02 & -0.17 & $\mathrm{dM}$ \\
\hline Gl 860 AB & -0.18 & 0.03 & -0.10 & -0.15 & -0.11 & $\mathrm{dM}$ \\
\hline G1 $234 \mathrm{AB}$ & -0.00 & 0.32 & 0.22 & $\ldots$ & 0.13 & $\mathrm{dM}$ \\
\hline G1 628 & -0.12 & 0.12 & -0.01 & -0.08 & -0.02 & $\mathrm{dM}$ \\
\hline Gl 687 & -0.13 & 0.12 & -0.02 & 0.04 & -0.09 & $\mathrm{dM}$ \\
\hline LHS 292 & $\ldots$ & $\ldots$ & 0.20 & $\ldots$ & -0.41 & $\mathrm{dM}$ \\
\hline GJ 1245 AC & $\ldots$ & 0.20 & 0.20 & $\ldots$ & -0.14 & $\mathrm{dM}$ \\
\hline GJ 1245 B & $\ldots$ & -0.07 & -0.18 & $\ldots$ & -0.13 & $\mathrm{dM}$ \\
\hline G1 876 & 0.01 & 0.38 & 0.22 & 0.02 & 0.18 & $\mathrm{dM}$ \\
\hline Gl 412 A & -0.52 & $\ldots$ & -0.50 & -0.33 & -0.40 & $\mathrm{dM}$ \\
\hline G1 388 & 0.03 & 0.37 & 0.20 & -0.21 & 0.28 & $\mathrm{dM}$ \\
\hline G1 873 & -0.10 & 0.17 & 0.03 & -0.21 & -0.01 & $\mathrm{dM}$ \\
\hline GJ $1116 \mathrm{AB}$ & $\ldots$ & $\ldots$ & 0.89 & $\ldots$ & -0.12 & $\mathrm{dM}$ \\
\hline GJ 3379 & -0.12 & 0.08 & -0.04 & $\ldots$ & 0.08 & $\mathrm{dM}$ \\
\hline Gl 445 & -0.30 & $\ldots$ & -0.34 & $\ldots$ & -0.25 & $\mathrm{dM}$ \\
\hline Gl 526 & -0.26 & -0.05 & -0.17 & 0.06 & -0.30 & $\mathrm{dM}$ \\
\hline LP 816-60 & -0.17 & -0.05 & -0.16 & $\ldots$ & 0.06 & $\ldots$ \\
\hline G1 251 & -0.23 & -0.03 & -0.16 & -0.13 & -0.07 & $\mathrm{dM}$ \\
\hline Gl 169.1 A & -0.01 & 0.34 & 0.20 & 0.06 & 0.36 & $\mathrm{dM}$ \\
\hline Gl 402 & $\ldots$ & -0.06 & -0.18 & -0.04 & 0.20 & $\mathrm{dM}$ \\
\hline G1 205 & -0.10 & 0.12 & $\ldots$ & -0.13 & 0.35 & $\mathrm{dM}$ \\
\hline G1 213 & $\ldots$ & -0.12 & -0.23 & $\ldots$ & -0.25 & $\mathrm{dM}$ \\
\hline Gl $752 \mathrm{AB}$ & -0.03 & 0.23 & 0.09 & 0.00 & -0.05 & $\mathrm{dM}$ \\
\hline Gl 285 & 0.09 & 0.55 & 0.40 & $\ldots$ & 0.40 & $\mathrm{dM}$ \\
\hline Gl 555 & 0.00 & 0.36 & 0.23 & 0.03 & 0.22 & $\mathrm{dM}$ \\
\hline Gl 338 A & -0.19 & $\ldots$ & $\ldots$ & -0.42 & -0.18 & $\operatorname{sdM}$ \\
\hline Gl 581 & -0.25 & -0.09 & -0.21 & -0.10 & -0.10 & $\mathrm{dM}$ \\
\hline G1 338 B & -0.34 & $\ldots$ & $\ldots$ & -0.29 & -0.15 & $\mathrm{dM}$ \\
\hline G1 $268 \mathrm{AB}$ & 0.17 & 0.76 & 0.65 & $\ldots$ & 0.10 & $\mathrm{dM}$ \\
\hline Gl 643 & $\ldots$ & $\ldots$ & -0.41 & -0.07 & -0.22 & $\mathrm{dM}$ \\
\hline G1 1156 & $\ldots$ & 0.07 & -0.01 & $\ldots$ & 0.15 & $\mathrm{dM}$ \\
\hline Gl 625 & -0.48 & $\ldots$ & -0.50 & -0.35 & -0.23 & $\mathrm{sdM}$ \\
\hline Gl 408 & -0.22 & -0.02 & -0.14 & -0.09 & -0.09 & $\mathrm{dM}$ \\
\hline GJ 1286 & $\ldots$ & -0.01 & -0.10 & $\ldots$ & -0.04 & $\mathrm{dM}$ \\
\hline G 203-47 & -0.08 & 0.19 & 0.06 & $\ldots$ & 0.33 & $\mathrm{dM}$ \\
\hline LHS 3376 & $\ldots$ & $\ldots$ & -0.68 & $\ldots$ & -0.12 & $\mathrm{dM}$ \\
\hline LHS 3799 & $\ldots$ & 0.20 & 0.14 & $\ldots$ & 0.38 & $\mathrm{dM}$ \\
\hline GJ 1224 & $\ldots$ & $\ldots$ & -0.29 & $\ldots$ & -0.05 & $\mathrm{dM}$ \\
\hline LHS 3558 & -0.18 & 0.04 & -0.10 & -0.14 & 0.01 & $\mathrm{dM}$ \\
\hline Gl 686 & -0.39 & $\ldots$ & -0.33 & -0.25 & -0.28 & $\mathrm{dM}$ \\
\hline GJ 3146 & $\ldots$ & $\ldots$ & $\ldots$ & $\ldots$ & -0.11 & $\mathrm{dM}$ \\
\hline LHS 2065 & $\ldots$ & $\ldots$ & $\ldots$ & $\ldots$ & 0.16 & $\mathrm{dM}$ \\
\hline G1 849 & 0.15 & 0.53 & 0.36 & $\ldots$ & 0.31 & $\mathrm{dM}$ \\
\hline LHS 224 & $\ldots$ & -0.06 & -0.18 & $\ldots$ & -0.46 & $\mathrm{dM}$ \\
\hline LHS 1809 & $\ldots$ & -0.10 & -0.25 & $\ldots$ & -0.01 & $\mathrm{dM}$ \\
\hline LHS 3549 & $\ldots$ & 0.05 & -0.05 & $\ldots$ & 0.16 & $\mathrm{dM}$ \\
\hline Gl 176 & -0.06 & 0.19 & 0.06 & -0.16 & 0.15 & $\mathrm{dM}$ \\
\hline LHS 495 & $\ldots$ & 0.13 & 0.03 & $\ldots$ & 0.20 & $\mathrm{dM}$ \\
\hline LHS 252 & $\ldots$ & 0.06 & -0.01 & $\ldots$ & -0.01 & $\mathrm{dM}$ \\
\hline$V^{*}$ V547 Cas & -0.26 & -0.05 & -0.18 & -0.18 & -0.28 & $\mathrm{dM}$ \\
\hline Gl 436 & -0.03 & 0.26 & 0.10 & -0.05 & 0.04 & $\mathrm{dM}$ \\
\hline GJ 1119 & $\ldots$ & 0.14 & 0.03 & $\ldots$ & -0.04 & $\mathrm{dM}$ \\
\hline Gl 649 & -0.15 & 0.07 & -0.05 & -0.13 & -0.04 & $\mathrm{dM}$ \\
\hline GJ 3147 & $\ldots$ & $\ldots$ & $\ldots$ & $\ldots$ & 0.21 & $\mathrm{dM}$ \\
\hline LHS 2924 & $\ldots$ & $\ldots$ & $\ldots$ & $\ldots$ & -0.19 & $\mathrm{dM}$ \\
\hline HIP 57050 & -0.02 & 0.31 & 0.15 & 0.07 & 0.05 & $\mathrm{dM}$ \\
\hline Gl 777 B & $\ldots$ & $\ldots$ & -0.39 & $\ldots$ & 0.03 & $\mathrm{dM}$ \\
\hline Gl 164 & -0.04 & 0.23 & 0.12 & . & 0.02 & $\mathrm{dM}$ \\
\hline
\end{tabular}

Table 7

(Continued)

\begin{tabular}{|c|c|c|c|c|c|c|}
\hline Name & $\begin{array}{c}\mathrm{B} 05 \\
{[\mathrm{Fe} / \mathrm{H}]}\end{array}$ & $\begin{array}{c}\text { JA09 } \\
{[\mathrm{Fe} / \mathrm{H}]}\end{array}$ & $\begin{array}{c}\text { SL10 } \\
{[\mathrm{Fe} / \mathrm{H}]}\end{array}$ & $\begin{array}{c}\text { W09 } \\
{[\mathrm{Fe} / \mathrm{H}]}\end{array}$ & $\begin{array}{c}\text { RA11 } \\
{[\mathrm{Fe} / \mathrm{H}]}\end{array}$ & $\begin{array}{c}\text { L07 } \\
\text { Class }\end{array}$ \\
\hline LHS 18 & $\ldots$ & $\ldots$ & $\ldots$ & $\ldots$ & -0.48 & $\mathrm{dM}$ \\
\hline Gl 179 & -0.01 & 0.32 & 0.16 & 0.02 & 0.23 & $\mathrm{dM}$ \\
\hline Gl 212 & -0.04 & 0.18 & 0.07 & -0.24 & 0.03 & $\mathrm{dM}$ \\
\hline LHS 3577 & -0.26 & -0.05 & -0.18 & -0.22 & -0.11 & $\mathrm{dM}$ \\
\hline GJ 1214 & $\ldots$ & 0.04 & -0.07 & $\ldots$ & 0.20 & $\mathrm{dM}$ \\
\hline LHS 220 & $\ldots$ & $\ldots$ & -0.72 & $\ldots$ & -0.35 & $\mathrm{dM}$ \\
\hline LHS 3605 & -0.51 & $\ldots$ & -0.57 & -0.19 & -0.28 & $\mathrm{dM}$ \\
\hline Gl 611 B & $\ldots$ & $\ldots$ & $\ldots$ & $\ldots$ & -0.45 & $\mathrm{dM}$ \\
\hline LHS 3593 & $\ldots$ & 0.03 & -0.08 & $\ldots$ & 0.03 & $\mathrm{dM}$ \\
\hline LHS 1706 & $\ldots$ & 0.23 & $\ldots$ & $\ldots$ & 0.21 & $\mathrm{dM}$ \\
\hline V* V492 Lyr & $\ldots$ & $\ldots$ & $\ldots$ & $\ldots$ & -0.04 & $\mathrm{dM}$ \\
\hline GJ 3253 & 0.05 & 0.47 & 0.38 & $\ldots$ & -0.07 & $\mathrm{dM}$ \\
\hline Gl 630.1 A & -0.11 & 0.15 & 0.02 & $\ldots$ & -0.39 & $\mathrm{dM}$ \\
\hline HIP 79431 & 0.19 & 0.56 & 0.39 & $\ldots$ & 0.46 & $\ldots$ \\
\hline V* V1513 Cyg & -0.89 & $\ldots$ & -1.02 & -0.80 & -0.64 & $\mathrm{sdM}$ \\
\hline LHS 494 & 0.08 & 0.49 & 0.32 & $\ldots$ & 0.24 & $\mathrm{dM}$ \\
\hline NSV 13261 & $\ldots$ & 0.31 & 0.26 & $\ldots$ & -0.29 & $\mathrm{dM}$ \\
\hline G 168-24 & -0.29 & $\ldots$ & -0.26 & -0.09 & 0.01 & $\mathrm{dM}$ \\
\hline LHS 3576 & -0.13 & $\ldots$ & -0.02 & -0.20 & -0.19 & $\mathrm{dM}$ \\
\hline LHS 3409 & $\ldots$ & $\ldots$ & $\ldots$ & $\ldots$ & -0.52 & sdM \\
\hline LHS 6007 & -0.04 & 0.27 & 0.14 & $\ldots$ & 0.16 & $\mathrm{dM}$ \\
\hline G 210-45 & -0.16 & 0.06 & -0.05 & -0.12 & -0.05 & $\mathrm{dM}$ \\
\hline HIP 12961 & -0.20 & $\ldots$ & -0.07 & $\ldots$ & 0.01 & $\ldots$ \\
\hline LHS 3591 & 0.06 & 0.35 & 0.21 & . & -0.12 & $\ldots$ \\
\hline G 262-29 & 0.16 & 0.38 & 0.28 & -0.19 & -0.30 & $\mathrm{dM}$ \\
\hline
\end{tabular}

Table 7 lists the ninety $\mathrm{M}$ dwarfs with Johnson- $V$ magnitudes and parallaxes, with their $[\mathrm{Fe} / \mathrm{H}]$ values obtained from the photometric calibrations and the $K$-band $[\mathrm{Fe} / \mathrm{H}]$ fit in Equation (7). Since the N12 photometric calibration is a marginal refinement of the Schlaufman \& Laughlin (2010) calibration, we only included the later in this comparison. Eight-six of the stars in Table 7 are also included in the Palomar-Michigan State University (PMSU) Nearby Star Spectroscopic Survey (Reid et al. 1997) and have measurements of the $\mathrm{TiO}$ and $\mathrm{CaH}$ feature strengths from their optical spectra. Woolf et al. (2009) created a spectroscopic optical technique based on these indices to estimate $[\mathrm{Fe} / \mathrm{H}]$ metallicities for early $\mathrm{M}$ dwarfs. The $[\mathrm{Fe} / \mathrm{H}]$ values predicted by the Woolf et al. (2009) technique from the PMSU indices are also listed in Table 7. The value of $[\mathrm{Fe} / \mathrm{H}]$ is listed for a star in Table 7 only if the parameters to calculate it satisfy the conditions of each technique.

1. Bonfils et al. (2005a, hereafter B05). The $[\mathrm{Fe} / \mathrm{H}]$ photometric relation by B05 is valid for stars with $4 \leqslant M_{K} \leqslant 7.5$, $2.5 \leqslant V-K_{s} \leqslant 6$, and $-1.5 \leqslant[\mathrm{Fe} / \mathrm{H}] \leqslant+0.2$.

2. Johnson \& Apps (2009, hereafter JA09). The [Fe/H] photometric relation by JA09 is valid for stars with $3.9 \leqslant V-K_{s} \leqslant 6.6$ and $[\mathrm{Fe} / \mathrm{H}] \geqslant-0.05$ dex. However, we decreased the $[\mathrm{Fe} / \mathrm{H}]$ lower limit to $-0.12 \mathrm{dex}$, which corresponds to the extrapolation done for the star Gl 832 in JA09.

3. Schlaufman \& Laughlin (2010, hereafter SL10). The $[\mathrm{Fe} / \mathrm{H}]$ photometric relation by SL10 does not explicitly mention any conditions. However, we only estimated $[\mathrm{Fe} / \mathrm{H}]$ values for stars with $3 \leqslant V-K_{s} \leqslant 7$, the color range in the color-magnitude diagrams in SL10.

4. Woolf et al. (2009, hereafter W09). The $[\mathrm{Fe} / \mathrm{H}]$ relation based on $\mathrm{TiO}$ and $\mathrm{CaH}$ molecular absorption by W09 depends on the $\zeta_{\mathrm{TiO} / \mathrm{CaH}}$ parameter by Lépine et al. 
(2007). The W09 calibration is valid for stars with $-1.5 \leqslant$ $[\mathrm{Fe} / \mathrm{H}] \leqslant+0.05$ and $3500 \mathrm{~K} \leqslant T_{\text {eff }} \leqslant 4000 \mathrm{~K}$. We used the stars with spectral types earlier than $\mathrm{M} 3$. The $\zeta_{\mathrm{TiO} / \mathrm{CaH}}$ parameter categorizes the main-sequence $M$ stars into four classes ordered by decreasing metallicity: dwarfs (dM), subdwarfs (sdM), extreme subdwarfs (esdM), and ultrasubdwarfs (usdM). The metallicity class for each star is also listed in Table 7.

Figure 13 shows the difference between the $[\mathrm{Fe} / \mathrm{H}]$ values obtained by Equation (7), and the metallicity methods listed before.

The B05 and W09 relations assign lower $[\mathrm{Fe} / \mathrm{H}]$ values to the stars with solar and super-solar metallicities according to the $K$-band $[\mathrm{Fe} / \mathrm{H}]$ calibration. The stars with $[\mathrm{Fe} / \mathrm{H}]$ differences within 0.15 dex have $K$-band $[\mathrm{Fe} / \mathrm{H}]$ metallicities between $\sim+0.1$ dex and $\sim-0.5$ dex, and correspond to $50 \%$ and $58 \%$ of the stars in the B05 and W07 samples, respectively.

The JA09 relation assigns higher $[\mathrm{Fe} / \mathrm{H}]$ values to the metalpoor stars in the $K$-band $[\mathrm{Fe} / \mathrm{H}]$ calibration. The stars with $0.15 \mathrm{dex}[\mathrm{Fe} / \mathrm{H}]$ differences (i.e., $\sim 1 \sigma$ of the $K$-band calibration) have $K$-band $[\mathrm{Fe} / \mathrm{H}]$ metallicities between -0.3 dex to +0.4 dex, and represent the $55 \%$ of the stars with JA09 metallicities.

The SL10 relation $[\mathrm{Fe} / \mathrm{H}]$ values agree within $0.15 \mathrm{dex}$ with the $K$-band $[\mathrm{Fe} / \mathrm{H}]$ calibration for $56 \%$ of the stars with SL10 metallicities. However, the dispersion for metalpoor stars is larger than for stars with high $K$-band $[\mathrm{Fe} / \mathrm{H}]$ values, when compared to the SL10 technique. The SL10 technique "underestimates" the values of some of the solar and super-solar $K$-band $[\mathrm{Fe} / \mathrm{H}]$ stars, but for low $K$-band $[\mathrm{Fe} / \mathrm{H}]$ values, it can either underestimate or overestimate that value by up to 0.6 dex. GJ $1116 \mathrm{~A}$ (M6) has a $K$-band metallicity of $[\mathrm{Fe} / \mathrm{H}]_{\mathrm{RA} 11}=-0.12 \mathrm{dex}$, but the SL10 method predicts a value of $[\mathrm{Fe} / \mathrm{H}]_{\mathrm{SL} 10}=+0.89$ dex. The SL10 $[\mathrm{Fe} / \mathrm{H}]$ value for GJ $1116 \mathrm{~A}$ is very unlikely and it may be a result of bad $V$ photometry or a bad parallax measurement.

All the plots in Figure 13 show a similar slope, except the $[\mathrm{Fe} / \mathrm{H}]_{\mathrm{SL} 10}-[\mathrm{Fe} / \mathrm{H}]_{\mathrm{RA} 11}$ versus $[\mathrm{Fe} / \mathrm{H}]_{\mathrm{RA} 11}$ plot. The W09 relation was calibrated using the $\zeta_{\mathrm{TiO} / \mathrm{CaH}}$ parameter and metallicities inferred from $\mathrm{Fe}$ I and $\mathrm{Ti}$ I abundances of K-dwarfs and early M dwarfs from their high resolution spectra (Woolf \& Wallerstein 2005). B05 acknowledge in their conclusion that their $[\mathrm{Fe} / \mathrm{H}]$ values are consistent (where the parameter spaces overlap) with the $[\mathrm{Fe} / \mathrm{H}]$ values from Woolf \& Wallerstein (2005). Since the B05 and W09 results agree, it is not surprising that both plots look quite similar. The JA09 calibration depends on the same observables as the B05 calibration, though super-solar metallicity stars were used as $[\mathrm{Fe} / \mathrm{H}]$ calibrators for the fit. This could explain why the JA09 plot has the same slope as the B05 and W09, but with an offset toward higher metallicities. The observables in B05 were also used in the SL10 calibration; however, the kinematics of the FGK-calibrators and theoretical models were considered in the SL10 calibration. For the super-solar metallicity stars, the SL10 shows just an offset toward lower metallicities when compared to the RA11 calibration. For the metal-poor stars, the scatter is larger but there is not a distinct slope between SL10 and RA11.

\subsection{Inferring Potential Optical Metallicity Indicators}

While M dwarfs are bright in the NIR, historically the optical regime has received more scrutiny as a means of studying $\mathrm{M}$ dwarfs. The molecules $\mathrm{CaH}, \mathrm{TiO}, \mathrm{CaOH}$, and $\mathrm{VO}$ cover the spectral range between 6300 and $9000 \AA$. TiO absorption bands are known to be metallicity dependent (e.g., Woolf \& Wallerstein 2006). Titanium oxide bands grow stronger with increasing metallicity as well as decreasing temperature. Hydride absorption bands also increase in strength with decreasing temperature, but, since the number density of neutral hydrogen increases as metallicity decreases, they grow stronger with decreasing abundance, too. Therefore, hydride absorption bands dominate the spectra of metal-poor stars, and the ratio between $\mathrm{TiO}$ and $\mathrm{CaH}$ has been used as a discriminant between disk and halo $\mathrm{M}$ stars (e.g., the subdwarf classification by Gizis 1997).

One hundred and nine $\mathrm{M}$ dwarfs studied in our sample are part of the PMSU Nearby Star Spectroscopic Survey (Reid et al. 1995; Hawley et al. 1996). The PMSU survey obtained optical spectroscopy of most of the M stars in The Third Catalogue of Nearby Stars (Gliese \& Jahreiss 1991), allowing spectral types to be estimated from $\mathrm{TiO}$ band-strengths, chromospherically active stars to be identified by $\mathrm{H}_{\alpha}$ emission, and metal-poor stars to be identified from the $\mathrm{CaH}$ strength relative to $\mathrm{TiO}$ strength. The overlap between our sample and the PMSU catalog allows us to test if an M dwarf's metallicity influences the spectral type obtained by the KHM technique.

Reid et al. (1995) defined 9 narrow band spectroscopic indices ( $5 \mathrm{TiO}$ indices, $3 \mathrm{CaH}$ indices, and $1 \mathrm{CaOH}$ index) to measure molecular features in the 6200-7200 $\AA$ wavelength range. Assuming that $\mathrm{TiO}$ band-strengths are primarily temperature dependent, Reid et al. (1995) measured the full strength of the $\mathrm{TiO}$ band at $\sim 7050 \AA$ (the TiO5 index) for a large number of stars with KHM spectral types to calibrate $\mathrm{TiO}$ as a spectral type indicator. Gizis (1997) used the Reid et al. (1995) index definitions to calibrate $\mathrm{CaH} 2$ and $\mathrm{CaH} 3$ indices as spectral type indicators, too. We calculated spectral types for the stars in our sample that are also in PMSU using the Reid et al. (1995) and Gizis (1997) fit relations. The spectral types derived from the $\mathrm{TiO} 5$ and $\mathrm{CaH}$ features agree for most of the sample. The difference in spectral type predicted by the optical indices and $\mathrm{H}_{2} \mathrm{O}-\mathrm{K} 2$ index as function of overall metallicity is shown in Figure 14. The TiO5 index predicts later subtypes than the $\mathrm{H}_{2} \mathrm{O}-\mathrm{K} 2$ index for the solar and metal-rich stars, and earlier subtypes for the metal-poor stars. The $\mathrm{CaH} 2$ index predicts later subtypes than the $\mathrm{H}_{2} \mathrm{O}-\mathrm{K} 2$ index for most of the stars. The $\mathrm{CaH} 3$ index tends to give earlier subtypes than the $\mathrm{H}_{2} \mathrm{O}-\mathrm{K} 2$ index. However, considering their uncertainties, the spectral types derived from the $\mathrm{TiO}$ and $\mathrm{CaH}$ indices agree with the $\mathrm{H}_{2} \mathrm{O}-\mathrm{K} 2$ index based spectral types.

The values of $\mathrm{TiO} 2, \mathrm{TiO} 3$, and $\mathrm{CaH} 1$ indices versus the $\mathrm{H}_{2} \mathrm{O}-\mathrm{K} 2$ index of the stars in the PMSU survey are shown in Figure 15. There is a strong correlation between the water index and the $\mathrm{TiO}$ indices up to $\mathrm{H}_{2} \mathrm{O}-\mathrm{K} 2 \sim 0.8$. For spectral types later than M6, VO decreases the strength of the TiO features at $\sim 7050 \AA$, and therefore, reverses the relation. The $\mathrm{CaH} 1$ index saturates at $\mathrm{TiO} 5 \sim 0.49$ and, hence, was not used as a spectral type indicator for the optical calibrations by Reid et al. (1995) and Gizis (1997). Reid et al. (1995) noticed that even when $\mathrm{CaH} 1$ was weaker that the $\mathrm{CaH} 3$ index, it appeared to be more sensitive to "(presumably) abundance variations among the earlier type $\mathrm{M}$ dwarfs." Figure 15 has $\mathrm{M}$ dwarfs with $[\mathrm{M} / \mathrm{H}]>0.0$ dex and $[\mathrm{M} / \mathrm{H}]<0.0$ dex color-coded with red and blue, respectively. The considerable dispersion in the $\mathrm{CaH} 1$ versus $\mathrm{H}_{2} \mathrm{O}-\mathrm{K} 2$ relation can be explained by the metallicities of the stars. The $\mathrm{CaH} 1$ index saturates at $\sim \mathrm{M} 3$, due to the increasing $\mathrm{TiO}$ absorption in that region of the optical spectrum. 

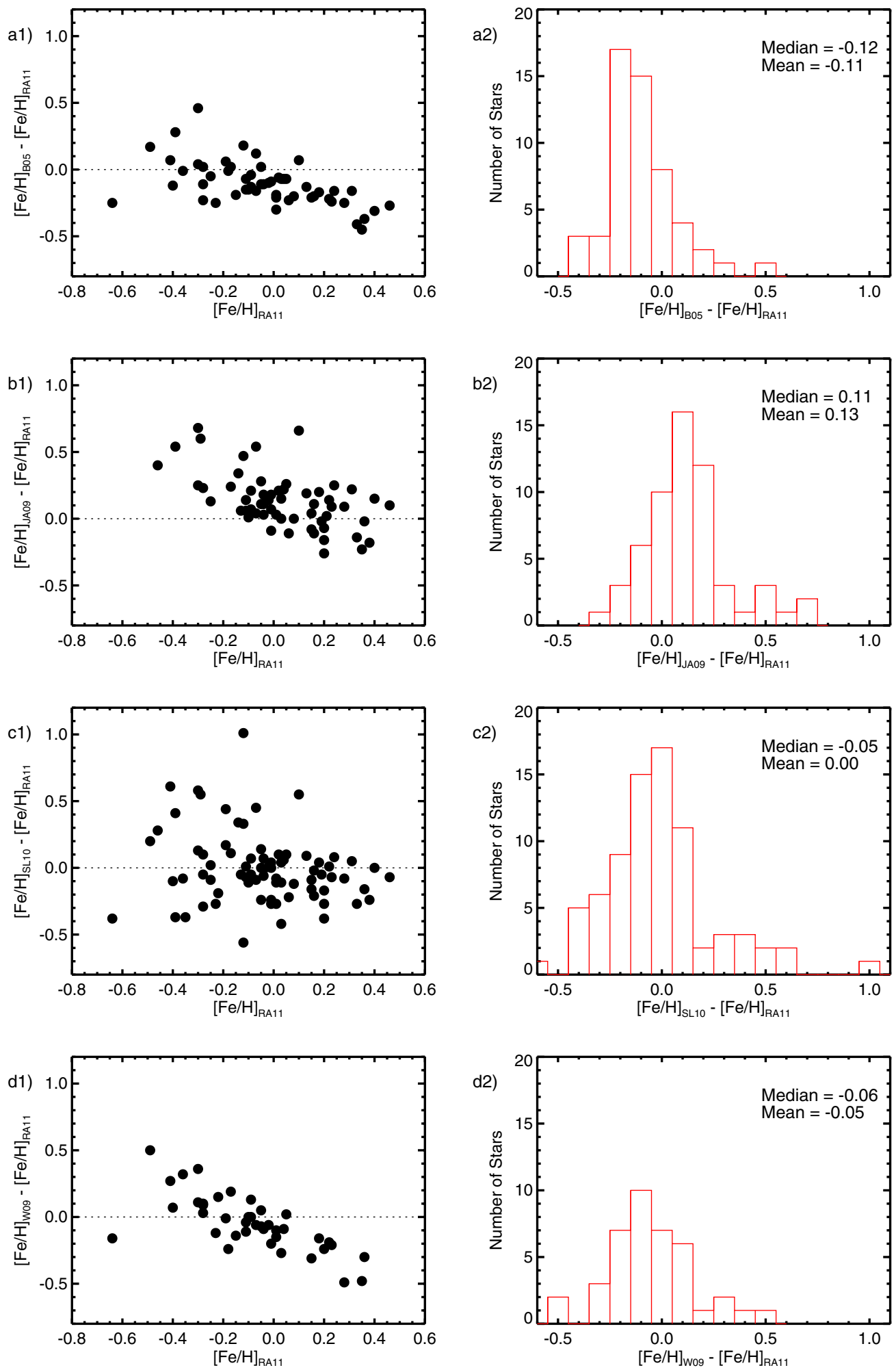

Figure 13. Differences between the $[\mathrm{Fe} / \mathrm{H}]$ values predicted for $\mathrm{M}$ dwarf stars in our sample from the $K$-band $[\mathrm{Fe} / \mathrm{H}]$ fit in Equation (7) and (a1) the [Fe/H] from $\mathrm{B} 05$, (b1) the $[\mathrm{Fe} / \mathrm{H}]$ from JA09, (c1) the $[\mathrm{Fe} / \mathrm{H}]$ from SL10, (d1) the $[\mathrm{Fe} / \mathrm{H}]$ from W09. The B05 and W09 calibrations underestimate $[\mathrm{Fe} / \mathrm{H}]$ values of the super-solar $K$-band $[\mathrm{Fe} / \mathrm{H}]$ stars. The JA09 calibration overestimates the $[\mathrm{Fe} / \mathrm{H}]$ values of the subsolar $K$-band $[\mathrm{Fe} / \mathrm{H}]$ stars. The scatter of the residuals increases for the subsolar $K$-band $[\mathrm{Fe} / \mathrm{H}]$ stars when their $[\mathrm{Fe} / \mathrm{H}]$ are compared with the SL10 $[\mathrm{Fe} / \mathrm{H}]$ values.

(A color version of this figure is available in the online journal.)

Since both molecules, $\mathrm{CaH}$ and $\mathrm{TiO}$, increase in strength toward lower temperatures, a ratio between these two molecules could be what $\mathrm{CaH} 1$ is really measuring. If a given star has low metallicity, the $\mathrm{TiO}$ absorption is not going to be as signif- icant as the $\mathrm{CaH}$, for any given temperature. The $\mathrm{CaH} 1$ feature will remain strong for low metallicities, which will correspond to low $\mathrm{CaH} 1$ index values. But, if the star is metal-rich, the pseudocontinuum used to estimate the $\mathrm{CaH} 1$ index is going to 

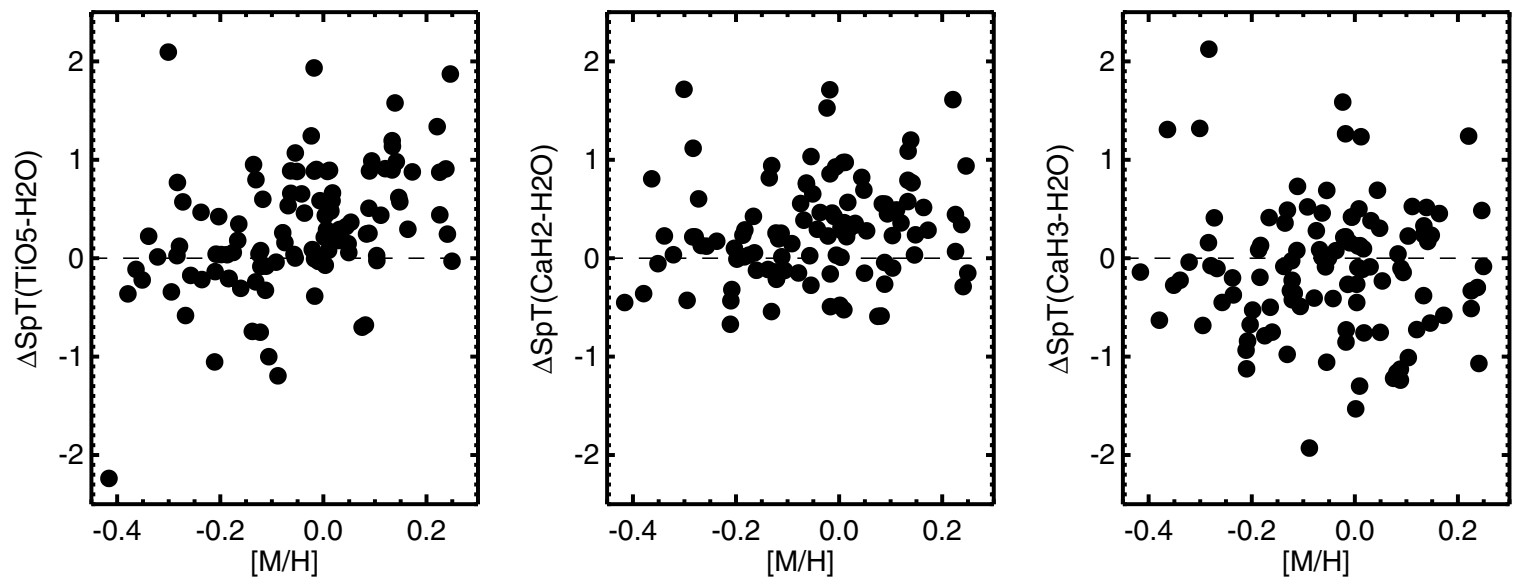

Figure 14. Difference between the spectral types derived using the $\mathrm{H}_{2} \mathrm{O}-\mathrm{K} 2$ index and the TiO5 (left), $\mathrm{CaH} 2$ (middle), and CaH3 (right) indices vs. [M/H]. The TiO5 index predicts later subtypes than the $\mathrm{H}_{2} \mathrm{O}-\mathrm{K} 2$ index for the solar and metal-rich stars, and earlier subtypes to the metal-poor stars. The CaH2 and CaH3 indices show less metallicity sensitivity. The $\mathrm{CaH} 2$ index predicts slightly later subtypes than the $\mathrm{H}_{2} \mathrm{O}-\mathrm{K} 2$ index, while the $\mathrm{CaH} 3$ index shows an opposite offset, but no bias with metallicity is visible for any of these two $\mathrm{CaH}$ indices.
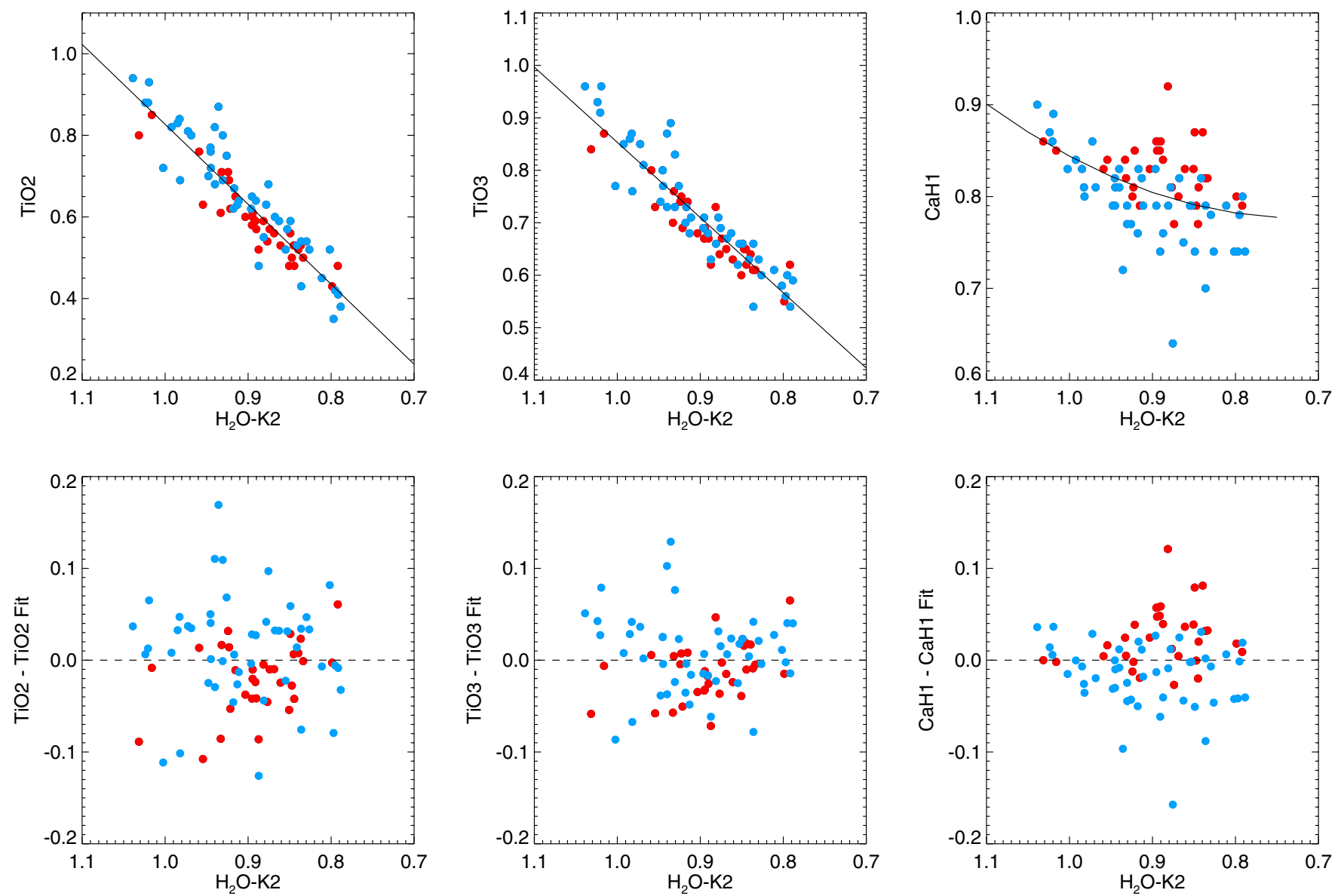

Figure 15. PMSU TiO2, TiO3, and $\mathrm{CaH} 1$ indices vs. the $\mathrm{H}_{2} \mathrm{O}-\mathrm{K} 2$ index. $\mathrm{M}$ dwarfs with $[\mathrm{M} / \mathrm{H}]>0.0$ dex and $[\mathrm{M} / \mathrm{H}]<0.0$ dex are color coded with red and blue, respectively. The TiO indices correlate with the $\mathrm{H}_{2} \mathrm{O}-\mathrm{K} 2$ index, with larger scatter toward early-type $\mathrm{M}$ dwarfs. The CaH1 index seems to be more sensitive to metallicity than any of the other indices.

(A color version of this figure is available in the online journal.)

be affected by the strong $\mathrm{TiO}$ absorption. The $\mathrm{CaH} 1$ feature will become less prominent, corresponding to high $\mathrm{CaH} 1$ index values. There is a similar split between the metal-rich and metal-poor $\mathrm{M}$ dwarfs for the $\mathrm{TiO} 2$ and $\mathrm{TiO} 3$ relations, but it is less significant. The $\mathrm{CaH} 1$ feature could be used as a metallicity discriminator for early solar-metallicity $\mathrm{M}$ dwarfs in the optical.

\section{INDEPENDENT TESTS OF THE $K$-BAND [Fe/H] FIT: M-M BINARIES, UVW SPACE MOTIONS, AND $\mathrm{H} \alpha$ ACTIVITY}

\subsection{Do M-M Binaries Return Similar Metallicities?}

The $K$-band $[\mathrm{Fe} / \mathrm{H}]$ calibration and the photometric methods rely on the assumption that stars in binary systems share the 
Table 8

M Dwarf Wide Binaries [Fe/H] Comparison

\begin{tabular}{|c|c|c|c|c|c|c|c|}
\hline \multirow[t]{2}{*}{ Name } & \multirow[t]{2}{*}{ Sp. Type } & \multicolumn{2}{|c|}{ This Work } & \multirow{2}{*}{$\begin{array}{c}\mathrm{B} 05 \\
{[\mathrm{Fe} / \mathrm{H}]}\end{array}$} & \multirow{2}{*}{$\begin{array}{c}\text { JA09 } \\
{[\mathrm{Fe} / \mathrm{H}]}\end{array}$} & \multirow{2}{*}{$\begin{array}{c}\text { SL10 } \\
{[\mathrm{Fe} / \mathrm{H}]}\end{array}$} & \multirow{2}{*}{$\begin{array}{c}\text { W09 } \\
{[\mathrm{Fe} / \mathrm{H}]}\end{array}$} \\
\hline & & {$[\mathrm{M} / \mathrm{H}]$} & {$[\mathrm{Fe} / \mathrm{H}]$} & & & & \\
\hline GJ 1245 AC & M6 & -0.09 & -0.14 & $\ldots$ & 0.20 & 0.20 & $\ldots$ \\
\hline GJ 1245 B & M6 & -0.09 & -0.13 & $\ldots$ & -0.07 & -0.18 & $\ldots$ \\
\hline Diff: & & 0.00 & 0.01 & & 0.26 & 0.3 & \\
\hline G1 338 A & M0 & -0.13 & -0.18 & -0.19 & $\ldots$ & $\ldots$ & -0.42 \\
\hline G1 338 B & M0 & -0.11 & -0.15 & -0.34 & $\ldots$ & $\ldots$ & -0.29 \\
\hline Diff: & & 0.02 & 0.03 & 0.15 & & & 0.13 \\
\hline G1 725 A & M1 & -0.34 & -0.49 & -0.32 & $\ldots$ & -0.29 & 0.01 \\
\hline Gl 725 B & M3 & -0.25 & -0.36 & -0.37 & $\ldots$ & -0.44 & -0.04 \\
\hline Diff: & & 0.09 & 0.13 & 0.05 & & 0.15 & 0.05 \\
\hline $\mathrm{V}^{*}$ V547 Cas & M2 & -0.19 & -0.28 & -0.26 & -0.05 & -0.18 & -0.18 \\
\hline LHS 115 & M3 & -0.19 & -0.27 & $\ldots$ & $\ldots$ & $\ldots$ & $\ldots$ \\
\hline Diff: & & 0.00 & 0.01 & & & & \\
\hline Gl 412 A & M1 & -0.28 & -0.40 & -0.52 & $\ldots$ & -0.50 & -0.33 \\
\hline Gl 412 B & M6 & -0.27 & -0.39 & $\ldots$ & $\ldots$ & $\ldots$ & $\ldots$ \\
\hline Diff: & & 0.01 & 0.01 & & & & \\
\hline Gl 643 & M3 & -0.15 & -0.22 & $\ldots$ & $\ldots$ & -0.41 & -0.07 \\
\hline Gl $644 \mathrm{C}$ & M7 & -0.21 & -0.32 & $\ldots$ & $\ldots$ & $\ldots$ & $\ldots$ \\
\hline Diff: & & 0.06 & 0.10 & & & & \\
\hline
\end{tabular}

same metallicity, as a result of being formed from the same interstellar material and at the same time. This assumption, which allows an M dwarf secondary's metallicity to be inferred from measurements of its FGK primary, has been validated for binaries with multiple solar-type components (Desidera et al. 2004, 2006), and implies that any metallicity estimation technique should return consistent values for two M dwarfs in a wide multiple system. As a sanity check, therefore, we inspect the $[\mathrm{Fe} / \mathrm{H}]$ values predicted by each technique for the individual components of double $\mathrm{M}$ dwarf wide binary systems in our sample. Table 8 shows the $K$-band and photometric metallicities of $5 \mathrm{M}-\mathrm{M}$ binary or multiple systems in our sample, along with their NIR spectral types; Gl 643 and Gl 644C are also included as a pair, since both are proper motion companions to the Gl 644AB pair/triplet according to Ségransan et al. (2000). Photometric estimates cannot be made for all the stars in the systems since their photometry and/or parallaxes do not satisfy the conditions listed in Section 5.3 for each calibration. We also show these systems in Figure 16, where we connect the individual components of a multiple system with dotted lines to examine how well each system agrees with the slope of our derived isometallicity contours.

The $K$-band $[\mathrm{Fe} / \mathrm{H}]$ and $[\mathrm{M} / \mathrm{H}]$ estimates for individual components of (non-calibrator) binary systems agree remarkably well; four of the six systems have $[\mathrm{Fe} / \mathrm{H}]$ and $[\mathrm{M} / \mathrm{H}]$ metallicity differences of less than 0.02 dex, and none of the six have individual metallicity estimates that disagree by more than 0.13 dex, comparable to the $1 \sigma$ accuracy of the underlying calibration itself. Indeed, the largest discrepancy for the $K$-band calibration is for the Gl 725AB pair, whose 0.09 dex and $0.13 \mathrm{dex}[\mathrm{M} / \mathrm{H}]$ and $[\mathrm{Fe} / \mathrm{H}]$ differences are similar to the dispersion (RMSE) of each fit in Equations (8) and (7), respectively.

The metallicity estimates predicted by the photometric techniques, however, do not reproduce this agreement: the photometric techniques predict metallicities for these individual binary components that typically differ by $0.1-0.2$ dex, comparable to the single largest metallicity difference predicted by the $K$-band technique. The Gl 412 system is the binary in our sample with the greatest spectral type difference, consisting of

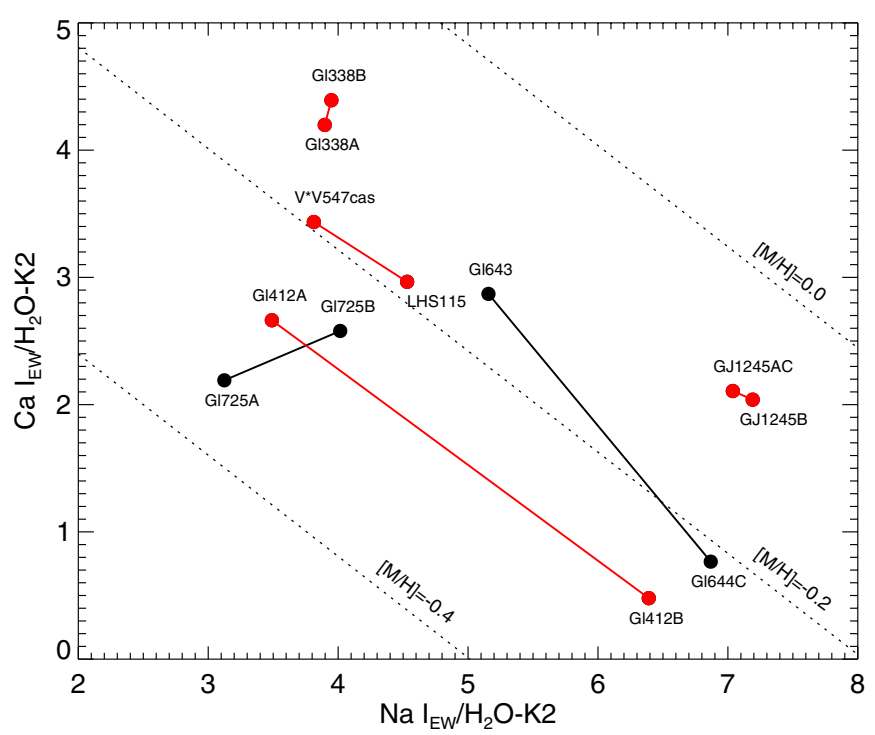

Figure 16. EW of $\mathrm{CaI}$ vs. EW of $\mathrm{NaI}$, weighted by $\mathrm{H}_{2} \mathrm{O}-\mathrm{K} 2$ index, for the $\mathrm{M}-\mathrm{M}$ binaries in Table 8 . The binary components are linked with lines. The dotted lines are isometallicity contours for $[\mathrm{M} / \mathrm{H}]$ calculated from Equation (8). The red dots/lines indicate the systems where the $K$-band metallicity estimates for the individual components agree to within 0.02 dex.

(A color version of this figure is available in the online journal.)

M1 and M6 components. Despite the significant range in temperatures between these two stars, the metallicities predicted by the $K$-band calibration only differ by $0.01 \mathrm{dex}$, suggesting that our calibration is not strongly biased with respect to $T_{\text {eff }}$. The GJ1245 system provides another good case study: both components have the same spectral-type in the optical and in the $K$ band, and their $K$-band spectra share a very similar morphology, so it is perhaps not surprising that the metallicity estimates predicted by the $K$-band calibration for the individual components agree quite well $(\Delta \mathrm{RA} 11[\mathrm{Fe} / \mathrm{H}]=0.01)$. The photometric $[\mathrm{Fe} / \mathrm{H}]$ estimates for each component, however, disagree much more $(\Delta[\mathrm{Fe} / \mathrm{H}]=0.25-0.3)$, reflecting underlying differences in the source photometry: while the distance measurements for 
GJ $1245 \mathrm{AC}$ and GJ $1245 \mathrm{~B}$ agree well $(\delta d=0.2 \mathrm{pc})$, their photometry does not, with GJ 1245 AC appearing $\sim 0.55 \mathrm{mag}$ brighter in both $V$ and $K_{s}$, placing it significantly higher in the $M_{V}$ versus $V-K_{s}$ diagram and thus implying a more metalrich composition via the photometric technique. McCarthy et al. (1988) resolved each component of the GJ 1245 triple system with speckle interferometry and obtained $K$-band photometry for each of its components. McCarthy et al.'s (1988) photometric measurements revealed almost identical magnitudes for the $\mathrm{A}$ and $\mathrm{B}$ components, $\sim 1.1$ mag brighter than $\mathrm{C}$. However, the combined $K$ magnitude for the GJ 1245 AC system was $\sim 0.5$ mag brighter than GJ $1245 \mathrm{~B}$, but the optical spectra for AC and B were almost identical. Therefore, the faint GJ $1245 \mathrm{C}$ component could explain the photometric $[\mathrm{Fe} / \mathrm{H}]$ discrepancy: it makes the star GJ 1245 A to appear brighter without affecting its optical and $K$-band spectra. This implies that an unknown unresolved binary could appear to be more metal-rich under the photometric calibration if it is assumed to be a single star.

\subsection{Do K-band Metallicities Reproduce the Metallicity/Kinematic Correlations Expected for Galactic Sub-populations?}

It has been known for the better part of a century that there are clear correlations between the spatial, kinematic, and chemical properties of stars in the Milky Way. These correlations allow the Milky Way to be decomposed into distinct stellar populations and structural components (e.g., halo versus disk; O'Connell 1958), although some debate remains concerning the exact number and properties of sub-components (e.g., thin versus thick disks; Ivezić et al. 2008). Bensby \& Feltzing (2010) recently showed that when 899 F- and G- dwarf stars were separated by their kinematic properties, a considerable number of thick disk stars exhibit metallicities consistent with the metallicity trend of the thin disk, and vice versa. When the solar-type stars were separated by age rather than kinematics, however, the number of outliers in each metallicity trend was reduced.

Age estimates for M dwarf stars are difficult to obtain, however, making kinematic properties the best available means for associating low-mass stars with various Galactic components. Bochanski et al. (2007) investigated metallicity differences between $\mathrm{M}$ dwarfs associated with the thin and thick disk populations, bisected kinematically using the method described by Bensby et al. (2003). Bochanski et al. (2007) measured the $(\mathrm{CaH} 2+\mathrm{CaH} 3) / \mathrm{TiO} 5$ ratio, previously shown by Lépine et al. (2003) to roughly discriminate between solar-metallicity $([\mathrm{M} / \mathrm{H}] \sim 0)$, subdwarf $([\mathrm{M} / \mathrm{H}] \sim-1.2)$, and extreme-subdwarf $([\mathrm{M} / \mathrm{H}] \sim-2.0) \mathrm{M}$ dwarfs, for $6577 \mathrm{M}$ dwarfs with Sloan Digital Sky Survey (SDSS) spectra. Bochanski et al. (2007) found that the observed $(\mathrm{CaH} 2+\mathrm{CaH} 3) / \mathrm{TiO} 5$ distributions for the kinematically divided thin and thick disk stars did not differ greatly, implying metallicity differences less than $1 \mathrm{dex}$, but suggested that the kinematically selected thick disk stars were consistent with an older population due to the lower fraction of stars with $\mathrm{H}_{\alpha}$ emission, a tracer of chromospheric activity.

One hundred and fourteen M dwarfs studied in this work have UVW space motions from the PMSU Nearby Star Spectroscopic Survey (Reid et al. 1997) enabling us to test if the $K$-band metallicity calibration can replicate the metallicity trends identified for higher-mass members of the kinematic sub-populations of the Milky Way. One way to visualize the different kinematic populations of the Milky Way is through the so-called Toomre diagram. ${ }^{13}$ The Toomre diagram is a representation of the stars' combined vertical and radial kinetic energies, $\left(\mathrm{U}^{2}+\mathrm{W}^{2}\right)^{1 / 2}$, as a function of the stars' rotational energy, $V$. Constant values of the total space velocity for the stars, $V_{\text {tot }}=\left(\mathrm{U}^{2}+\mathrm{W}^{2}+\mathrm{V}^{2}\right)^{1 / 2}$, can be represented by circles centered at $[0,0]$, as is shown in steps of $50 \mathrm{~km} \mathrm{~s}^{-1}$ in Figure 17. We base our kinematic sub-divisions on the boundaries defined by Bensby \& Feltzing (2010), where thin disk stars possess $V_{\text {tot }}<50 \mathrm{~km} \mathrm{~s}^{-1}$ and thick disk stars possess $\sim 70 \mathrm{~km} \mathrm{~s}^{-1}<V_{\text {tot }}<200 \mathrm{~km} \mathrm{~s}^{-1}$; we additionally classify stars with intermediate velocities $\left(50 \mathrm{~km} \mathrm{~s}^{-1}<V_{\text {tot }}<70 \mathrm{~km} \mathrm{~s}^{-1}\right)$ as thin-thick stars. We show in Figure 18 the distributions of $K$-band overall metallicity estimates for members of each of these kinematics groups, with the mean and median $[\mathrm{M} / \mathrm{H}]$ indicated for each distribution. Consistent with results seen for higher mass stars, we find the thin disk population is noticeably enriched $(\Delta[\mathrm{M} / \mathrm{H}] \sim 0.2$ dex $)$ relative to the thick disk population, and a two-sided K-S test identifies a $<0.003 \%$ probability that the two samples are consistent with the same parent distribution.

To further explore the differences between these populations, we show in Figure 17 the Toomre diagram for the stars with kinematic measurements in the PMSU survey, color-coded by metallicity, with boundaries for the metallicity sub-groups chosen from the mean values of the metallicity distributions of Figure 18. Visualizing the sample in this way, it is apparent that the metal-poor stars span a much larger range of velocities than the metal-rich stars: more than half the stars with $[\mathrm{M} / \mathrm{H}]<-0.14$ have $V_{\text {tot }}>70 \mathrm{~km} \mathrm{~s}^{-1}$, while every star with $[\mathrm{M} / \mathrm{H}]>-0.15$ lies within the $V_{\text {tot }} \sim 100 \mathrm{~km} \mathrm{~s}^{-1}$ contour. These results reinforce the trends seen by Bensby \& Feltzing (2010) for higher mass stars: Bensby \& Feltzing (2010) found that older $(t>8 \mathrm{Gyr})$ stars are a kinematically hot sample that cover most of the velocity space in the Toomre diagram, just like the thickdisk M dwarfs in Figure 17. Bensby \& Feltzing (2010) also found kinematically hot stars within the younger $(t<7 \mathrm{Gyr})$ population, but the majority have $V_{\text {tot }} \leqslant 100 \mathrm{~km} \mathrm{~s}^{-1}$, consistent with what we see in Figure 17 for our (metallicity selected) thin-disk M dwarfs.

\subsection{Are K-band Metallicities Consistent with an Assumed Age-Metallicity Activity Relation?}

A final observable that can be used to probe the existence of an underlying age-metallicity relation is chromospheric activity. Common tracers of chromospheric activity, such as emission in $\mathrm{Ca}$ II $\mathrm{H} \& \mathrm{~K}$ or $\mathrm{H}_{\alpha}$, indicate that young stars demonstrate high levels of chromospheric activity, with the activity decaying as the stars' age; this effect has been seen in solar-type stars (Skumanich 1972) and well into the M dwarf regime (Stauffer \& Hartmann 1986). Chromospheric activity timescales of $\mathrm{M}$ dwarfs expand from $1 \mathrm{Gyr}$ for early-type/higher-mass stars to $\sim 10 \mathrm{Gyr}$ for the later-type/lower-mass stars (Hawley et al. 1999; West et al. 2008).

As noted in the previous section, Bensby \& Feltzing (2010) found that the link between age and metallicity appears to be stronger for solar-type stars than the link between kinematics and metallicity. While it is not possible to derive an exact age for an $\mathrm{M}$ dwarf based on its observed activity level, one can use the activity lifetimes noted above to statistically divide stars into older and younger samples. Gizis et al. (2002) measured the EWs of $\mathrm{H}_{\alpha}$ on high-resolution spectra of $676 \mathrm{M}$ dwarfs

\footnotetext{
13 Sandage \& Fouts (1987) were the first to show this diagram in their paper on the collapse of the Galaxy, but attributed the diagram to A. Toomre.
} 


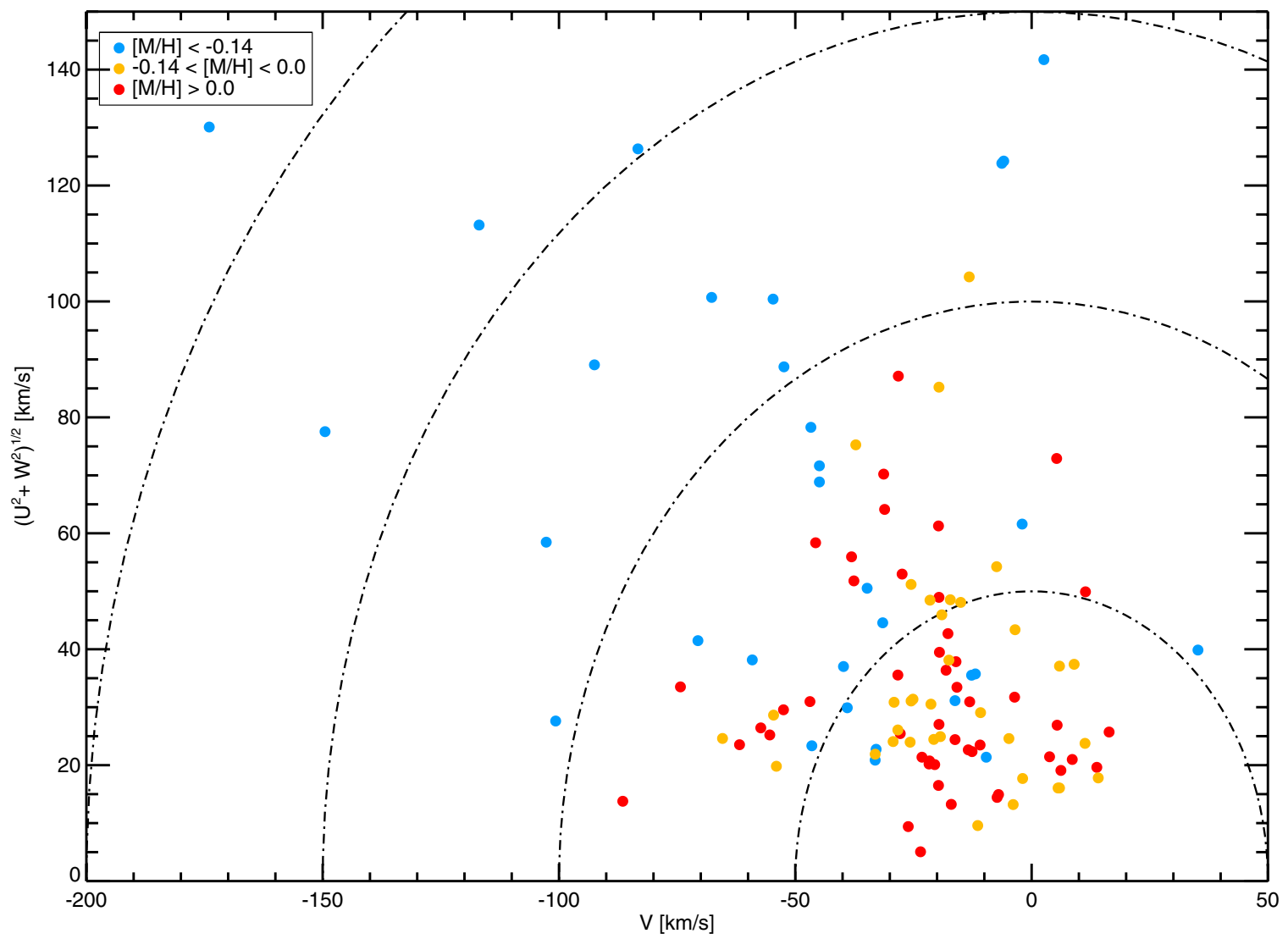

Figure 17. Toomre diagram for the stars in our sample with PMSU kinematics, color-coded by metallicity. The metal-poor stars $([\mathrm{Fe} / \mathrm{H}]<-0.14$ dex $)$, depicted by blue dots, cover the whole velocity space, while the metal-rich stars $\left([\mathrm{Fe} / \mathrm{H}]>0.0\right.$ dex) exhibit $V_{\text {tot }}$ velocities no larger than $\sim 100 \mathrm{~km} \mathrm{~s}^{-1}$.

(A color version of this figure is available in the online journal.)
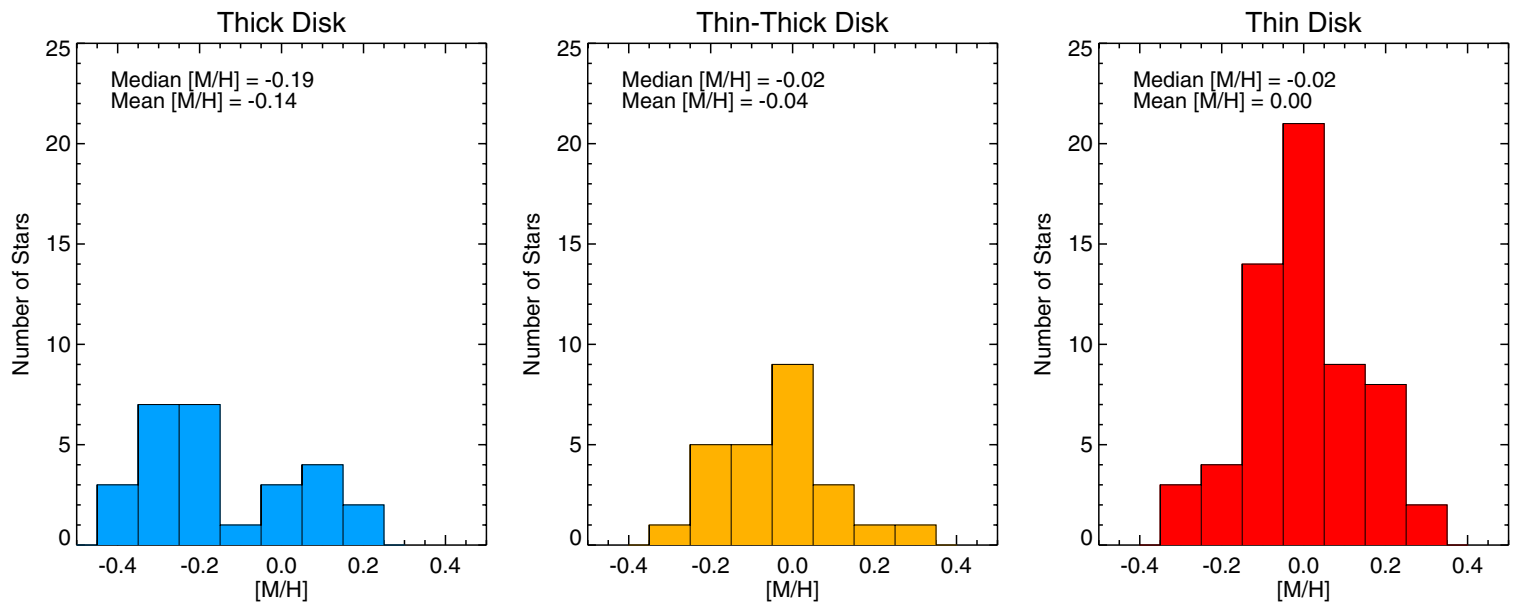

Figure 18. $K$-band metallicity distributions for the stars in our sample with PMSU kinematics. The boundaries of these kinematic sub-groups are: thin disk, $V_{\text {tot }}<$ $50 \mathrm{~km} \mathrm{~s}^{-1}$; thin-thick, $50 \mathrm{~km} \mathrm{~s}^{-1}<V_{\text {tot }}<70 \mathrm{~km} \mathrm{~s}^{-1}$; thick disk, $70 \mathrm{~km} \mathrm{~s}^{-1}<V_{\mathrm{tot}}<200 \mathrm{~km} \mathrm{~s}^{-1}$. The metallicity distributions of these kinematically selected subgroups are consistent with the trends seen for solar-type stars: stars with large space motions are preferentially metal-poor, while stars with small space motions are preferentially metal-rich.

(A color version of this figure is available in the online journal.)

and identified distinct groups according to their $\mathrm{H}_{\alpha}$ properties. Our sample includes 82 stars analyzed by Gizis et al. (2002), providing an opportunity to use activity as a crude proxy for age, and examine the evidence for an age-metallicity relationship within the $\mathrm{M}$ dwarfs in our sample.

Figure 19 shows the $\mathrm{H}_{\alpha}$ emission strength versus spectral type for these stars, divided according to the metallicity boundaries inferred from Figure 18 and applied previously in Figure 17.
Across all metallicity categories, most of the early-type (M0 to M2) stars show $\mathrm{H}_{\alpha}$ absorption, implying either a weak/moderate activity or no activity at all, while the later-type stars show $\mathrm{H}_{\alpha}$ emission, consistent with the longer activity lifetimes expected for the lowest-mass stars. The metal-rich category seems to have a slightly higher average level of $\mathrm{H}_{\alpha}$ emission when compared with the other categories, consistent with the metal-rich stars being a statistically younger sample than the more metal-poor 

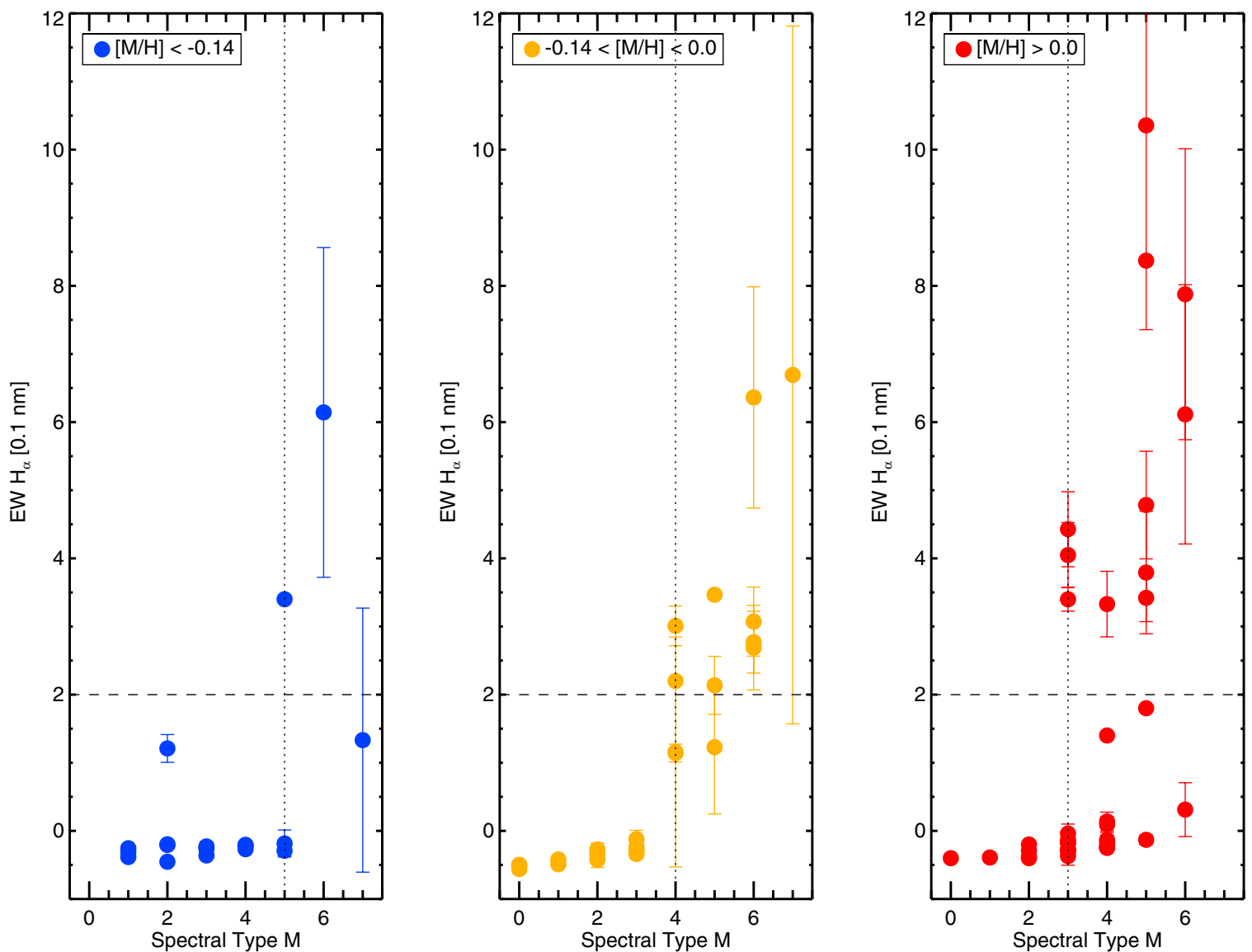

Figure 19. $\mathrm{H}_{\alpha}$ strength as a function of spectral type for the stars in our sample with $\mathrm{H}_{\alpha}$ measurements by Gizis et al. (2002). The dotted-line represents a $2 \AA$ lower limit in $\mathrm{H}_{\alpha}$ to define $\mathrm{M}$ dwarfs with significantly enhanced activity (i.e., more active than required to satisfy the $0.75 \AA \AA$ Aimit adopted by West et al. 2008). All late-type (>M5) stars are still active in all three metallicity categories, but activity persists to earlier types in the metal-rich samples, consistent with an underlying correlation between a star's age, activity, and metallicity. Chromospheric activity is noticeable for M3 stars with $[\mathrm{M} / \mathrm{H}]>-0.01$ dex, for M4 stars with -0.15 dex $<[\mathrm{M} / \mathrm{H}]<-0.01$ dex, and for an M5 stars with $[\mathrm{M} / \mathrm{H}]<-0.15$ dex. $\mathrm{V}^{*} \mathrm{~V} 1513 \mathrm{Cyg}$ is a metal-poor M2 dwarf that still exhibits chromospheric activity.

(A color version of this figure is available in the online journal.)

samples. If we adopt a $2 \AA$ threshold for significant $\mathrm{H}_{\alpha}$ activity, the earliest star in each category with elevated activity is clearly correlated with metallicity: the earliest active metal-poor star is an M5, while the earliest active intermediate and metal-rich stars are M4 and M3, respectively. This correlation is precisely what would be expected if there were an underlying age-metallicity relation, such that the sample of metal-poor stars consists of statistically older stars, where activity is only seen for the lowermass stars with relatively long activity lifetimes, while metalrich stars are statistically young, with higher mass stars not yet having transitioned into the inactive stage.

\section{PARTICULARLY PROMISING M DWARF PLANET HOSTS}

The nearly 600 confirmed extrasolar planets discovered to date reveal that planets are a natural and frequent by-product of star formation. One of the most important observational constraints is that planet frequency rises steeply with host star abundance above solar metallicity in FGK stars (e.g., Santos et al. 2004; Fischer \& Valenti 2005). As mentioned in the introduction, the first photometric and spectroscopic metallicity measurements for $\mathrm{M}$ dwarf planet hosts indicated that they were slightly metal-poor (Bonfils et al. 2005a; Bean et al. 2006a). However, recent metallicity calibrations (Johnson \& Apps 2009; Rojas-Ayala et al. 2010; Schlaufman \& Laughlin 2010) have convincingly shown that the small sample of nearby M dwarf planet hosts have solar and super-solar metallicities.

Table 10 flags the $\mathrm{M}$ dwarf planet hosts in our sample. According to the metallicity calibration derived in Section 5.2, the $11 \mathrm{M}$ dwarf planet hosts have overall metallicities higher than -0.06 dex, with the Jovian hosts being more metal-rich than the Neptune or super-Earth hosts. The sample of M dwarf planet hosts is small, but their $K$-band metallicities suggest the existence of a planet-metallicity correlation for $\mathrm{M}$ dwarfs. We compared the $[\mathrm{Fe} / \mathrm{H}]$ distribution of the 11 planet hosts to the $[\mathrm{Fe} / \mathrm{H}]$ distribution of $35 \mathrm{M}$ dwarfs in our sample that are also members of the California Planet Survey (CPS) sample of lowmass stars (J. Johnson 2011, private communication). These $35 \mathrm{M}$ dwarfs have been subjected to an intensive radial velocity (RV) monitoring campaign by the CPS team, and their RV measurements have ruled out the presence of Jupiter-size planets within several AU. Figure 20 shows the metallicity distributions of the 35 CPS M dwarfs and of the 11 planet hosts in our sample. A two-sided K-S test identifies that there is a $<1.6 \%$ probability that the $K$-band metallicities we calculate for these samples of $\mathrm{M}$ dwarfs with and without planets share the same parent distribution. If the planet sample is divided by Jupiter and Neptune/super-Earth hosts, then a two-sided K-S test gives a $0.8 \%$ chance that the Jovian hosts and the 35 CPS stars are drawn from the same parent distribution. The probability that the Neptune and super-Earth hosts share the same 


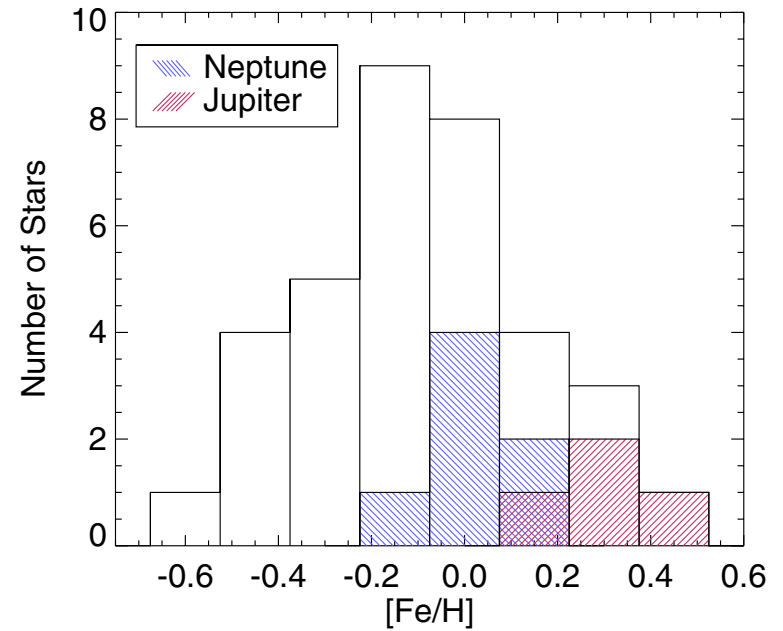

Figure 20. $[\mathrm{Fe} / \mathrm{H}]$ distribution of $35 \mathrm{M}$ dwarfs whose RV measurements have rule out the presence of Jupiter-size planets within several AU by the CPS team, along with the $[\mathrm{Fe} / \mathrm{H}]$ distribution of Jupiter hosts and Neptune hosts in our sample.

(A color version of this figure is available in the online journal.)

$[\mathrm{Fe} / \mathrm{H}]$ distribution as the $35 \mathrm{CPS}$ stars without detected planets is much higher: $8.6 \%$. These results are in agreement with that observed for FGK stars, where Neptunes and super-Earths, unlike Jupiters, do not seem to form preferentially around highly metal-rich $([\mathrm{Fe} / \mathrm{H}]>0.2 \mathrm{dex})$ stars (Sousa et al. 2008; Mayor et al. 2011). However, we acknowledge that the small size of these samples prevents any conclusive result.

According to the $[\mathrm{Fe} / \mathrm{H}]$ distribution of the planet hosts in our sample, M dwarfs with solar and super-solar metallicities should be the preferred targets to look for planets around cool stars. The most metal-rich stars $([\mathrm{M} / \mathrm{H}]>+0.2 \mathrm{dex})$ in this sample are the Jovian hosts HIP 79431 and Gl 849, and the M dwarfs Gl 285 (M5), G 203-47 (M4), Gl 169.1 A (M3), LHS 3799 (M5), and
G1 205 (M0). Bower et al. (2009) used radio astrometry to search for planets around a sample of active $\mathrm{M}$ stars within $10 \mathrm{pc}$ and their analyzed sample included two of the metal-rich stars listed above (Gl 285 and LHS 3799). Bower et al. (2009) were able to rule out the existence of 3-6 $M_{J}$ planets within 1 AU at the $99 \%$ confidence level. Lawler et al. (2009) searched for an infrared excess around G1 205 and found none excluding the presence of debris at distances beyond the snow line.

Interestingly, the metal-rich stars G 203-47 and Gl 169.1 A do not have planetary companions, but they do possess white dwarf (WD) companions. Gl 169.1 B is a relatively young and featureless WD with an age estimate of $\sim 2.8$ Gyr based on a model with a core composed of pure carbon (Leggett et al. 1998). Gl 203-47 B does not have an age estimate, however Delfosse et al. (1999) predicted that its primary (Gl 203-47) would have a peculiar composition since the system was likely in a contact configuration during the post main-sequence evolution of the current day WD component. The semi-major axis of the present orbit of the G 203-47 system is $\sim 0.05$ AU (Delfosse et al. 1999). Therefore, G 203-47 could have accreted nucleosynthesis products dredged up to the surface of Gl 203-47B's asymptotic giant branch progenitor. Figure 21 shows the optical PMSU spectra and $K$-band spectra of G 203-47 and Gl 169.1 A, along with the spectra of LHS 3409, a star with similar spectral type but subsolar metallicity. The strong $\mathrm{Na}$ I and $\mathrm{Ca}$ I features in $K$ band, and the weak $\mathrm{CaH}$ absorption in their optical spectra, indicate that these stars have indeed super-solar metallicities.

Most of exoplanet searches have been performed at visible wavelengths. M dwarfs are intrinsically faint in the optical, so the largest stellar component in the solar neighborhood have not yet been searched thoroughly for planets, except for the brightest, closest early $\mathrm{M}$ dwarfs. However, planet searches are now being performed with near-infrared RV instruments (e.g., Bean et al. 2010; Crockett et al. 2011; Muirhead et al. 2011) and with far red filters for transits (Irwin et al. 2009). For these searches, the visible faintness of $\mathrm{M}$ dwarfs is not a problem, but
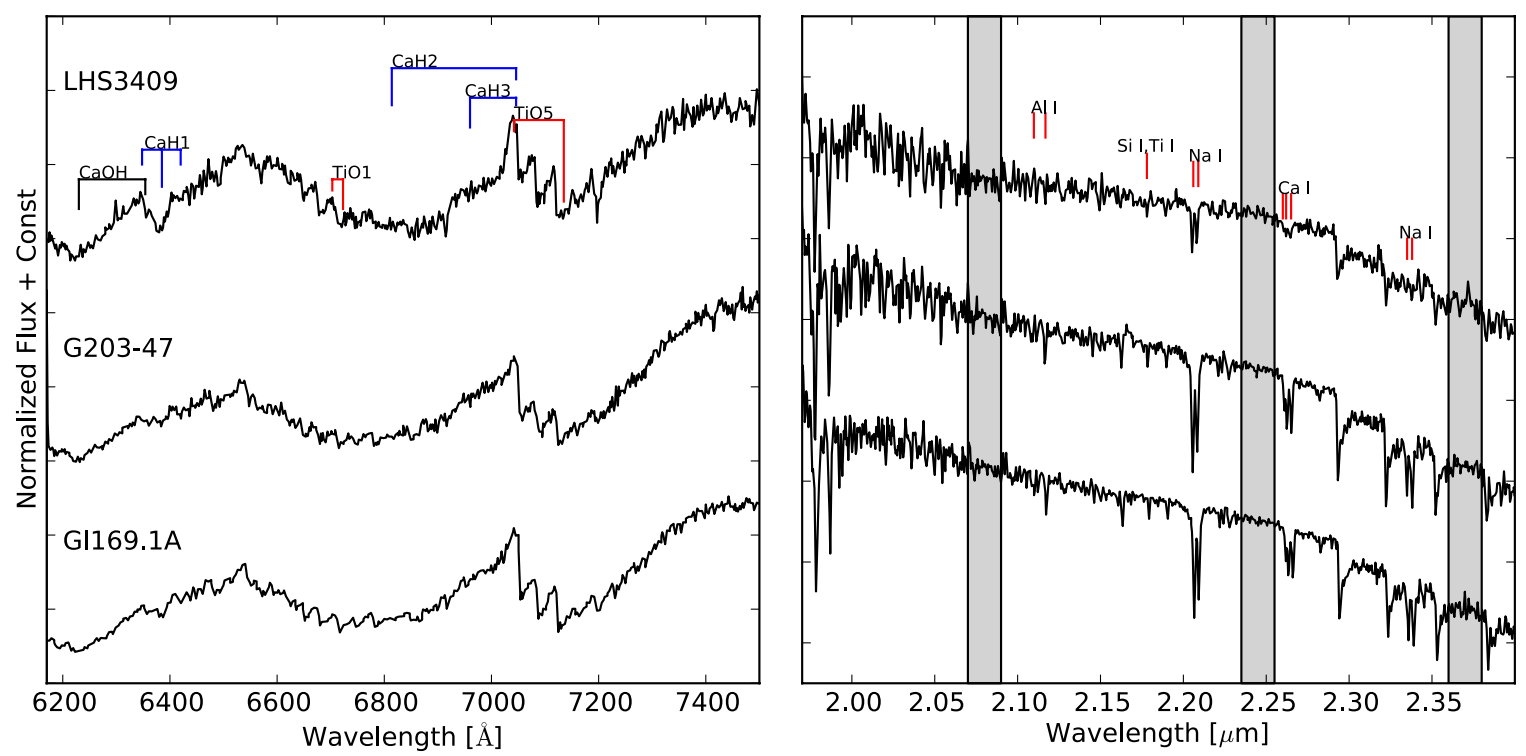

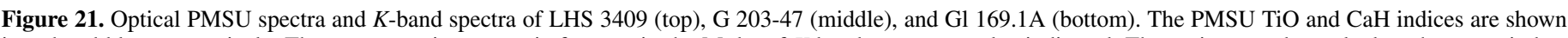

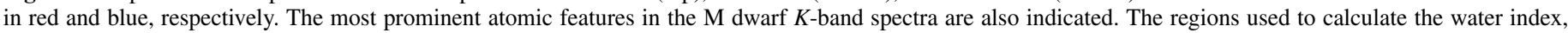

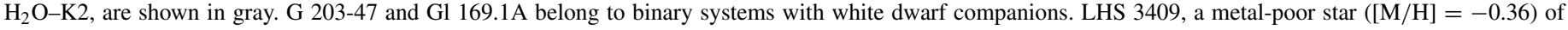

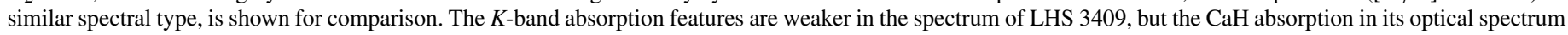
is stronger than in the two metal-rich stars, which indicates that G 203-47 and G1 169.1A have super-solar metallicities.

(A color version of this figure is available in the online journal.) 
Table 9

M Dwarf Planet Hosts and Metallicity Calibration Sample

\begin{tabular}{|c|c|c|c|c|c|c|c|c|c|c|c|}
\hline \multirow[t]{2}{*}{ Name } & \multicolumn{3}{|c|}{ This Work } & \multirow{2}{*}{$\begin{array}{c}\text { KHM } \\
\text { Sp. Type }\end{array}$} & \multirow{2}{*}{$\begin{array}{c}\mathrm{B} 05 \\
{[\mathrm{Fe} / \mathrm{H}]}\end{array}$} & \multirow{2}{*}{$\begin{array}{c}\text { JA09 } \\
{[\mathrm{Fe} / \mathrm{H}]}\end{array}$} & \multirow{2}{*}{$\begin{array}{c}\text { W09 } \\
{[\mathrm{Fe} / \mathrm{H}]}\end{array}$} & \multirow{2}{*}{$\begin{array}{c}\text { SL10 } \\
{[\mathrm{Fe} / \mathrm{H}]}\end{array}$} & \multirow{2}{*}{$\begin{array}{c}\mathrm{R} 10 \\
{[\mathrm{Fe} / \mathrm{H}]}\end{array}$} & \multirow{2}{*}{$\begin{array}{c}M \sin (i) \\
M_{J}\end{array}$} & \multirow[t]{2}{*}{ Planet Notes } \\
\hline & Sp. Type & {$[\mathrm{M} / \mathrm{H}]$} & {$[\mathrm{Fe} / \mathrm{H}]$} & & & & & & & & \\
\hline HIP 79431 & M1 & +0.33 & +0.46 & M3 & +0.16 & +0.52 & $\ldots$ & +0.35 & +0.60 & 2.1 & Jupiter \\
\hline G1 849 & M2 & +0.23 & +0.31 & M3 & +0.14 & +0.58 & +0.20 & +0.41 & +0.49 & 0.82 & Jupiter \\
\hline Gl 179 & M3 & +0.17 & +0.23 & M3.5 & $\ldots$ & +0.30 & +0.02 & +0.20 & $\ldots$ & 0.82 & Jupiter \\
\hline GJ 1214 & M4 & +0.15 & +0.20 & M4.5 & $\ldots$ & +0.03 & $\ldots$ & +0.28 & +0.39 & 0.0179 & Super-Earth \\
\hline G1 876 & M3 & +0.14 & +0.19 & M4 & +0.03 & +0.37 & +0.02 & +0.23 & +0.43 & 2.64 & 2 Jupiters + Neptune + super-Earth \\
\hline Gl 176 & M2 & +0.11 & +0.15 & M2.5 & $\ldots$ & +0.18 & -0.16 & +0.06 & $\ldots$ & 0.0265 & Super-Earth \\
\hline Gl 436 & M3 & +0.04 & +0.05 & M2 & -0.03 & +0.25 & -0.05 & +0.10 & -0.00 & 0.072 & Neptune \\
\hline HIP 57050 & M3 & +0.03 & +0.04 & M4 & -0.02 & +0.32 & +0.07 & $\ldots$ & +0.12 & 0.298 & Neptune \\
\hline HIP 12961 & M1 & +0.01 & +0.01 & M0 & $\ldots$ & $\ldots$ & $\ldots$ & -0.07 & $\ldots$ & 0.35 & Neptune \\
\hline Gl 649 & M1 & -0.02 & -0.04 & M1 & -0.18 & +0.04 & -0.13 & -0.03 & +0.14 & 0.328 & Neptune \\
\hline Gl 581 & M2 & -0.06 & -0.10 & M3 & -0.25 & -0.10 & -0.10 & -0.22 & -0.02 & 0.0492 & Neptune +3 super-Earths \\
\hline
\end{tabular}

Table 10

Best M Dwarf Targets for Planet Searches

\begin{tabular}{|c|c|c|c|c|c|c|c|c|c|}
\hline Name & PMSU & Sp. Type & $V$ & $K_{s}$ & {$[\mathrm{M} / \mathrm{H}]$} & {$[\mathrm{Fe} / \mathrm{H}]$} & $\mathrm{H}_{\alpha}{ }^{1}$ & $v \sin i^{2}$ & Pop \\
\hline Gl 581 & 2420 & M2 & 10.57 & 5.84 & -0.06 & -0.10 & -0.359 & $\leqslant 2.1^{\mathrm{a}}$ & Thin \\
\hline Gl 687 & 2797 & M3 & 9.15 & 4.55 & -0.06 & -0.09 & -0.34 & $\leqslant 2.8^{\mathrm{a}}$ & Thin \\
\hline Gl 408 & 1709 & M2 & 10.03 & 5.50 & -0.06 & -0.09 & -0.307 & $\leqslant 2.3^{\mathrm{a}}$ & Thin \\
\hline G1 251 & 1094 & M2 & 9.9 & 5.3 & -0.04 & -0.07 & -0.272 & $\leqslant 2.4^{\mathrm{a}}$ & Thin \\
\hline G1 $752 \mathrm{AB}$ & 3037 & M1 & 9.12 & 4.67 & -0.03 & -0.05 & -0.42 & $<2.5^{\mathrm{b}}$ & Thin/Thick \\
\hline Gl 649 & 2673 & M1 & 9.7 & 5.6 & -0.02 & -0.04 & -0.49 & $\leqslant 1.9^{\mathrm{c}}$ & Thin \\
\hline Gl 628 & 2599 & M3 & 10.1 & 5.08 & -0.01 & -0.02 & -0.234 & $1.1^{\mathrm{d}}$ & Thin \\
\hline Gl 447 & 1849 & M4 & 11.12 & 5.65 & -0.00 & -0.01 & -0.181 & $\leqslant 2^{\mathrm{e}}$ & Thin \\
\hline LHS 1809 & 1004 & M4 & 14.5 & 8.4 & -0.00 & -0.01 & -0.231 & $4.3^{\mathrm{f}}$ & Thin \\
\hline LHS 252 & 1355 & M6 & 15.05 & 8.67 & 0.00 & -0.01 & 0.312 & $4^{\mathrm{f}}$ & Thin/Thick \\
\hline LHS 3558 & 3203 & M3 & 10.4 & 5.9 & 0.01 & 0.01 & -0.039 & $\leqslant 3.2^{\mathrm{a}}$ & Thin \\
\hline Gl250 B & 1092 & M2 & 10.1 & 5.7 & 0.01 & 0.01 & -0.292 & $<2.5^{\mathrm{b}}$ & Thin \\
\hline G1 212 & 956 & M1 & 9.9 & 5.8 & 0.02 & 0.03 & -0.391 & $<2.5^{\mathrm{b}}$ & Thin \\
\hline G1 436 & 1830 & M3 & 10.7 & 6.1 & 0.03 & 0.04 & -0.37 & $\leqslant 1^{\mathrm{c}}$ & Thin/Thick \\
\hline LHS 3549 & 3195 & M5 & 14.0 & 8.1 & 0.12 & 0.16 & -0.131 & $\leqslant 4.5^{\mathrm{f}}$ & Thin/Thick \\
\hline G1 905 & 3743 & M4 & 12.3 & 5.9 & 0.14 & 0.19 & 0.092 & $\leqslant 1.2^{\mathrm{a}}$ & Thick \\
\hline G1 876 & 3604 & M2 & 10.2 & 5.0 & 0.14 & 0.19 & -0.2 & $\leqslant 2^{\mathrm{e}}$ & Thin \\
\hline Gl 402 & 1687 & M3 & 11.7 & 6.4 & 0.15 & 0.20 & -0.28 & $\leqslant 2.3^{\mathrm{e}}$ & Thin \\
\hline Gl 555 & 2310 & M3 & 11.3 & 5.9 & 0.16 & 0.22 & -0.177 & $2.7^{\mathrm{e}}$ & Thin \\
\hline G1 324 B & 1383 & M4 & 13.2 & 7.7 & 0.22 & 0.31 & -0.249 & $<2.5^{\mathrm{b}}$ & Thin \\
\hline G1 849 & 3478 & M2 & 10.4 & 5.6 & 0.23 & 0.31 & -0.384 & $<2.5^{\mathrm{b}}$ & Thin \\
\hline Gl 205 & 930 & M0 & 8.0 & 4.0 & 0.25 & 0.35 & -0.401 & $1^{\mathrm{d}}$ & Thin/Thick \\
\hline Gl 169.1 A & 780 & M3 & 11.1 & 5.7 & 0.26 & 0.36 & -0.174 & $1.9^{\mathrm{e}}$ & Thin/Thick \\
\hline
\end{tabular}

Notes.

${ }^{1}$ Reference of $\mathrm{H}_{\alpha}$ : Gizis et al. 2002.

${ }^{2}$ Reference of $v$ sin $i$ : (a) Delfosse et al. 1998b; (b) Browning et al. 2010; (c) Marcy \& Chen 1992; (d) Reiners 2007; (e) Mohanty \& Basri 2003; and (f) Jenkins et al. 2009.

other intrinsic properties of $\mathrm{M}$ dwarfs should be considered to optimize detectability.

Therefore, the most "desirable" $\mathrm{M}$ dwarf targets for planet searches should be:

1. Bright. The optical faintness of $\mathrm{M}$ dwarfs is not a problem for the near-infrared searches, but a large number of $\mathbf{M}$ dwarfs per night is desirable. Brighter $\mathbf{M}$ stars are favorable for achieving a fixed $\mathrm{S} / \mathrm{N}$ and for ease of followup for any planets that are detected.

2. Slow rotators. Stars with high rotation rates have RV measurements with lower precision since stellar rotation widens the spectral features. Bouchy et al. (2001) found that the RV uncertainties increase by a factor of $\sim 4$ when the rotation velocity increases from $\sim 1 \mathrm{~km} \mathrm{~s}^{-1}$ to $10 \mathrm{~km} \mathrm{~s}^{-1}$. Stars with $v \sin i>10 \mathrm{~km} \mathrm{~s}^{-1}$ should be avoided to provide optimal RV sensitivity.
3. Inactive. The activity of $\mathrm{M}$ dwarfs can also cause false positives (e.g., starspot-crossing events; Carter et al. 2011) and can cause RV and photometric jitter (Wright 2005; Berta et al. 2011). Depending on the starspot coverage, the planet-to-star radius ratio can change up to few percent (Carter et al. 2011). Stars with $\mathrm{H}_{\alpha}$ emission should be avoided to avoid false positives and jitter.

4. Metal-rich. The M dwarf planet hosts shown in Table 9 all have metallicities $[\mathrm{M} / \mathrm{H}] \geqslant-0.05$ dex. Therefore, it is probable that solar and super-solar metallicity $\mathrm{M}$ dwarfs are more likely to host planets.

Table 10 lists the stars that satisfy the conditions mentioned above for activity, rotation, and metallicity in our $\mathrm{M}$ dwarf sample. The $\mathrm{H}_{\alpha}$ values are from Gizis et al. (2002). The $v$ $\sin i$ values were taken from Jenkins et al. (2009, Tables 1 and 3 ) and Browning et al. (2010). Some of the stars in Table 10 
already have detected stellar or planetary companions (five of the eleven $\mathrm{M}$ dwarf planet hosts have their $\mathrm{H}_{\alpha}$ and $v$ sin $i$ listed in the papers mentioned above). It will be interesting to see how many planets are discovered around the targets in Table 10 in the near future, and what "flavor" they might be.

\section{CONCLUSIONS}

We present $K$-band spectra for a sample of $133 \mathrm{M}$ dwarfs, including $18 \mathrm{M}$ dwarfs with reliable metallicity estimates (as inferred from an FGK type companion), 11 planet hosts, more than $2 / 3$ of the Northern 8 pc sample, and additional $\mathrm{M}$ dwarfs from the Lepine Shara Catalog. From these spectra, we measured EWs of the $\mathrm{CaI}$ and $\mathrm{NaI}$ lines, as well as an index quantifying the absorption due to $\mathrm{H}_{2} \mathrm{O}$ opacity, features we have previously shown to be of use for predicting $\mathrm{M}$ dwarf temperatures and metallicities.

1. From the subset of our stars that possess primary or secondary KHM spectral types, we calibrated the $\mathrm{H}_{2} \mathrm{O}-\mathrm{K} 2$ index as a spectral type indicator. We also estimated effective temperatures for the stars in the sample by interpolating their $\mathrm{H}_{2} \mathrm{O}-\mathrm{K} 2$ indices onto a solar metallicity $\left[\mathrm{H}_{2} \mathrm{O}-\mathrm{K} 2\right.$, $\left.T_{\text {eff }}\right]$ model grid.

2. We revise the functional form adopted to predict the $[\mathrm{Fe} / \mathrm{H}]$ metallicities of $\mathrm{M}$ dwarfs based on measurements of their $\mathrm{Na}$ I, Ca I, and $\mathrm{H}_{2} \mathrm{O}$ features. We perform a linear regression upon $18 \mathrm{M}$ dwarfs with reliable metallicity estimates from FGK companions to calibrate this updated relationship. Statistical tests demonstrate that the $[\mathrm{Fe} / \mathrm{H}]$ estimates produced by this relationship are accurate to RMSE = $0.141 \mathrm{dex}$, and confirm that this relation accounts for more of the variance within our calibrant sample than any other existing technique for estimating $\mathrm{M}$ dwarf metallicities.

3. For the first time, we derive an expression for an M dwarf's overall metallicity $[\mathrm{M} / \mathrm{H}]$; quantitative comparisons to model atmospheres, which are benchmarked according to overall metallicity, provide a qualitative validation of our approach for estimating metallicities from $\mathrm{NaI}$, Ca I, and $\mathrm{H}_{2} \mathrm{O}$ features, but also demonstrate an overall offset between the atomic line strengths predicted by models as compared to actual observations.

4. We examined previous optical molecular indices sensitive to stellar metallicity using out metallicity estimates. We identify the $\mathrm{CaH} 1$ feature as a potentially valuable optical metallicity discriminator for solar-metallicity early-type M dwarfs.

5. We perform several sanity checks of our metallicity estimates, confirming that our metallicity estimates reproduce expected correlations between metallicity and Galactic space motions and $\mathrm{H} \alpha$ emission line strengths, and return statistically identical metallicities for members of $\mathrm{M}-\mathrm{M}$ multiple systems.

6. We recovered the results from previous metallicity studies that nearby $\mathrm{M}$ dwarf planet host exhibits solar to super-solar metallicities, where stars with Jovian-mass planets are more metal-rich than stars with Neptune-likes or super-Earths. A list of the best targets in the sample for planetary searches selected by metallicity, activity, and rotation-rates is given.

We thank the staff and telescope operators of Palomar Observatory for their support. We thank Travis Barman, John Bochanski, Jeff Valenti, and Andrew West for helpful discussions about various topics. We thank John Johnson for providing the $\mathrm{M}$ dwarf targets in the CPS target list for the K-S test calculations. We thank the anonymous referee for her/his helpful comments that improved our manuscript.

K.R.C. acknowledges support for this work from the Hubble Fellowship Program, provided by NASA through Hubble Fellowship grant HST-HF-51253.01-A awarded by the STScI, which is operated by the AURA, Inc., for NASA, under contract NAS 5-26555.

This research has made use of NASA's Astrophysics Data System Bibliographic Services, the SIMBAD database, operated at CDS, Strasbourg, France, the NASA/IPAC Extragalactic Database, operated by the Jet Propulsion Laboratory, California Institute of Technology, under contract with the National Aeronautics and Space Administration, and the VizieR database of astronomical catalogs (Ochsenbein et al. 2000).

This publication makes use of data products from the Two Micron All Sky Survey, which is a joint project of the University of Massachusetts and the Infrared Processing and Analysis Center/California Institute of Technology, funded by the National Aeronautics and Space Administration and the National Science Foundation.

Facility: Hale (TSPEC)

\section{APPENDIX}

\section{STATISTICAL TESTS AND RELIABLE UNCERTAINTIES FOR FUNCTIONAL FITS}

A well-fitting regression model results in predicted values close to the observed data values. The following statistics are commonly used to evaluate a model fit.

Residual mean square (RMSp). The RMSp is defined as

$$
\mathrm{RMSp}=\sum_{i=1}^{n} \frac{\left(\widehat{\mathrm{y}}_{\mathrm{i}}-\mathrm{y}_{\mathrm{i}}\right)^{2}}{n-p},
$$

where $n$ is the number of data points (in this case the number of calibrators), $p$ is the number of predictors in the model (including the constant term), $y_{i}$ is the value of the response variable $i$, and $\widehat{y}_{i}$ is the prediction value given by the regression model for $y_{i}$. The RMSp is the variance of the residuals. In general, an RMSp value closer to 0 indicates a model fit that is more useful for prediction.

Root-mean-square error (RMSE). The RMSE is the square root of the variance of the residuals

$$
\text { RMSE }=\sqrt{\text { RMSp. }}
$$

The RMSE indicates how accurately the regression model predicts the response. The RMSE is an absolute measure of it and has the same units as the response variable. Lower values of RMSE indicate better fit.

Adjusted square of the multiple correlation coefficient $\left(R_{a p}^{2}\right)$. The $R_{\text {ap }}^{2}$ represents the proportion of variability in a data set that is accounted for by a regression model. The $R_{\text {ap }}^{2}$ is defined as

$$
\begin{aligned}
R_{a p}^{2} & =1-\frac{(n-1) \sum_{i=1}^{n}\left(\widehat{y}_{i}-y_{i}\right)^{2}}{(n-p) \sum_{i=1}^{n}\left(y_{i}-\bar{y}\right)^{2}} \\
& =1-\frac{\operatorname{RMSp}}{\mathrm{MST}},
\end{aligned}
$$

where $\bar{y}$ is the overall mean of the observations $y_{i}$, and MST is the total mean of squares. The $R_{\text {ap }}^{2}$ compares the unbiased 
variance of the residuals (RMSp) and the unbiased variances of the observations (MST), therefore, can be interpreted as the proportion of total variance that is explained by the model. A value of $R_{\text {ap }}^{2}=1$ indicates that the regression model explains all of the variance in the sample, while $R_{\mathrm{ap}}^{2}=0$ indicates that the regression models explain none of the variance. $R_{\text {ap }}^{2}$ should always be used with models with more than one predictor variable.

Root-mean-square error of validation $\left(R M S E_{V}\right)$. A better estimate of the uncertainty in the $K$-band $[\mathrm{Fe} / \mathrm{H}]$ fit can be obtained by performing the Predicted Residual Sum of Squares (PRESS) procedure (Weisberg 2005). The PRESS statistic is equivalent to the "leave-one-out" method, where, in this case, the regression procedure is repeated leaving one of the $n$ calibrators out and performing the regression with the other $n-1$ calibrators, for each one of the $n$ calibrators. The PRESS statistics is defined as

$$
\begin{aligned}
\operatorname{PRESS} & =\sum_{i=1}^{n} \widehat{e}_{i}^{2} \\
& =\sum_{i=1}^{n}\left(\widehat{y}_{\left(-c_{i}\right) i}-y_{i}\right)^{2}
\end{aligned}
$$

where $\widehat{e}_{i}$ is the residual for the calibrator $c_{i}$, computed as the difference between the observed value of the predictand $y_{i}$ and the prediction $\widehat{y}_{\left(-c_{i}\right) i}$ from a regression model calibrated using the calibration sample but without the calibrator $c_{i}$. Then, it is possible to use as a measure of uncertainty a statistic based on this validation data

$$
\operatorname{RMSE}_{\mathrm{V}}=\sqrt{\frac{\mathrm{PRESS}}{n_{V}}} .
$$

Equation (A6) is known as the root-mean-squared error of validation $\left(\mathrm{RMSE}_{V}\right)$, where $n_{V}$ is the number of calibrators. The $\mathrm{RMSE}_{V}$ value is a sensible estimate of average prediction error according to Weisberg (2005). The $\mathrm{RMSE}_{V}$ value can be used then to obtain confidence intervals at a desired significance level around the predictions

$$
\widehat{y}_{i} \pm \mathrm{C}\left(t_{\alpha / 2, n-p}\right) \mathrm{RMSE}_{\mathrm{V}},
$$

where the multiplier of $\mathrm{RMSE}_{V}$ corresponds to the desired probability point on the cumulative distribution function. The correct cumulative distribution function for this specific fit is the two-sided $t$-student distribution with $n-p=15$ degrees of freedom, where $n=18$ is the sample size for calibration and $p=3$ is the number of regressors in the model fit, including the constant term. The two-sided $t$-student distribution is preferred instead of a normal distribution due to the small number of calibrators $\left(n=n_{V}=18\right)$. For approximate $70 \%, 95 \%$, and $99 \%$ confidence intervals, the values for $\mathrm{C}\left(t_{\alpha / 2, n-p=15}\right)$ are equal to $1.07,2.13$, and 2.95 , respectively.

\section{REFERENCES}

Ali, B., Carr, J. S., Depoy, D. L., Frogel, J. A., \& Sellgren, K. 1995, AJ, 110 , 2415

Allard, F. 1990, PhD Thesis, Ruprecht Karls Univ., Heidelberg

Allard, F., \& Hauschildt, P. H. 1995, ApJ, 445, 433

Allard, F., Hauschildt, P. H., \& Schwenke, D. 2000, ApJ, 540, 1005

Allard, F., Homeier, D., \& Freytag, B. 2010, arXiv e-prints

Apps, K., Clubb, K. I., Fischer, D. A., et al. 2010, PASP, 122, 156
Asplund, M., Grevesse, N., Sauval, A. J., \& Scott, P. 2009, ARA\&A, 47, 481

Bastian, N., Covey, K. R., \& Meyer, M. R. 2010, ARA\&A, 48, 339

Bean, J. L., Benedict, G. F., \& Endl, M. 2006a, ApJ, 653, L65

Bean, J. L., Seifahrt, A., Hartman, H., et al. 2010, ApJ, 713, 410

Bean, J. L., Sneden, C., Hauschildt, P. H., Johns-Krull, C. M., \& Benedict, G. F. 2006b, ApJ, 652, 1604

Bensby, T., \& Feltzing, S. 2010, in IAU Symp. 265, Chemical Abundances in the Universe: Connecting First Stars to Planets, ed. K. Cunha, M. Spite, \& B. Barbuy (Cambridge: Cambridge Univ. Press), 300

Bensby, T., Feltzing, S., \& Lundström, I. 2003, A\&A, 410, 527

Berta, Z. K., Charbonneau, D., Irwin, J., et al. 2011, BAAS, 43, 343.11

Bessel, M. S. 1990, A\&AS, 83, 357

Bochanski, J. J., Munn, J. A., Hawley, S. L., et al. 2007, AJ, 134, 2418

Bonfils, X., Delfosse, X., Udry, S., et al. 2005a, A\&A, 442, 635

Bonfils, X., Forveille, T., Delfosse, X., et al. 2005b, A\&A, 443, L15

Bouchy, F., Pepe, F., \& Queloz, D. 2001, A\&A, 374, 733

Bower, G. C., Bolatto, A., Ford, E. B., \& Kalas, P. 2009, ApJ, 701, 1922

Browning, M. K. 2008, ApJ, 676, 1262

Browning, M. K., Basri, G., Marcy, G. W., West, A. A., \& Zhang, J. 2010, AJ, 139,504

Butler, R. P., Johnson, J. A., Marcy, G. W., et al. 2006, PASP, 118, 1685

Butler, R. P., Vogt, S. S., Marcy, G. W., et al. 2004, ApJ, 617, 580

Carter, J. A., Winn, J. N., Holman, M. J., et al. 2011, ApJ, 730, 82

Charbonneau, D., Berta, Z. K., Irwin, J., et al. 2009, Nature, 462, 891

Covey, K. R., Lada, C. J., Román-Zúñiga, C., et al. 2010, ApJ, 722, 971

Crockett, C. J., Mahmud, N. I., Prato, L., et al. 2011, ApJ, 735, 78

Cushing, M. C., Rayner, J. T., \& Vacca, W. D. 2005, ApJ, 623, 1115

Delfosse, X., Forveille, T., Beuzit, J.-L., et al. 1999, A\&A, 344, 897

Delfosse, X., Forveille, T., Mayor, M., et al. 1998a, A\&A, 338, L67

Delfosse, X., Forveille, T., Perrier, C., \& Mayor, M. 1998b, A\&A, 331, 581

Delfosse, X., Forveille, T., Ségransan, D., et al. 2000, A\&A, 364, 217

Demory, B.-O., Ségransan, D., Forveille, T., et al. 2009, A\&A, 505, 205

Desidera, S., Gratton, R. G., Lucatello, S., \& Claudi, R. U. 2006, A\&A, 454 581

Desidera, S., Gratton, R. G., Scuderi, S., et al. 2004, A\&A, 420, 683

Fernandez, J. M., Latham, D. W., Torres, G., et al. 2009, ApJ, 701, 764

Fischer, D. A., \& Valenti, J. 2005, ApJ, 622, 1102

Förster Schreiber, N. M. 2000, AJ, 120, 2089

Forveille, T., Bonfils, X., Delfosse, X., et al. 2009, A\&A, 493, 645

Forveille, T., Bonfils, X., Lo Curto, G., et al. 2011, A\&A, 526, A141

Gizis, J. E. 1997, AJ, 113, 806

Gizis, J. E., Reid, I. N., \& Hawley, S. L. 2002, AJ, 123, 3356

Gliese, W., \& Jahreiss, H. 1991, NASA STI/Recon Technical Report A, 92, 33932

Gonzalez, G. 1997, MNRAS, 285, 403

Gould, A., \& Chanamé, J. 2004, ApJS, 150, 455

Haghighipour, N., Vogt, S. S., Butler, R. P., et al. 2010, ApJ, 715, 271

Hawley, S. L., Gizis, J. E., \& Reid, I. N. 1996, AJ, 112, 2799

Hawley, S. L., Reid, I. N., Gizis, J. E., \& Byrne, P. B. 1999, in ASP Conf. Ser 158, Solar and Stellar Activity: Similarities and Differences, ed. C. J. Butler \& J. G. Doyle (San Francisco, CA: ASP), 63

Henry, T. J., Jao, W.-C., Subasavage, J. P., et al. 2006, AJ, 132, 2360

Herter, T. L., Henderson, C. P., Wilson, J. C., et al. 2008, Proc. SPIE, 7014, 30

Horne, K. 1986, PASP, 98, 609

Howard, A. W., Johnson, J. A., Marcy, G. W., et al. 2010, ApJ, 721, 1467

Irwin, J., Charbonneau, D., Nutzman, P., \& Falco, E. 2009, in IAU Symp. 253,

Transiting Planets, ed. F. Pont, D. Sasselov, \& M. Holman (Cambridge:

Cambridge Univ. Press), 37

Irwin, J. M., Quinn, S. N., Berta, Z. K., et al. 2011, ApJ, 742, 123

Ivezić, Ž., Sesar, B., Jurić, M., et al. 2008, ApJ, 684, 287

Jenkins, J. S., Ramsey, L. W., Jones, H. R. A., et al. 2009, ApJ, 704, 975

Johnson, J. A., \& Apps, K. 2009, ApJ, 699, 933

Johnson, J. A., Howard, A. W., Marcy, G. W., et al. 2010, PASP, 122, 149

Jones, H. R. A., Longmore, A. J., Jameson, R. F., \& Mountain, C. M. 1994, MNRAS, 267, 413

Jones, H. R. A., Pavlenko, Y., Viti, S., et al. 2005, MNRAS, 358, 105

Kelly, B. C. 2007, ApJ, 665, 1489

Kirkpatrick, J. D., Henry, T. J., \& McCarthy, D. W., Jr. 1991, ApJS, 77, 417

Kleinmann, S. G., \& Hall, D. N. B. 1986, ApJS, 62, 501

Koen, C., Kilkenny, D., van Wyk, F., \& Marang, F. 2010, MNRAS, 403, 1949

Kraus, A. L., Tucker, R. A., Thompson, M. I., Craine, E. R., \& Hillenbrand, L. A. 2011, ApJ, 728, 48

Lawler, S. M., Beichman, C. A., Bryden, G., et al. 2009, ApJ, 705, 89

Leggett, S. K. 1992, ApJS, 82, 351

Leggett, S. K., Ruiz, M. T., \& Bergeron, P. 1998, ApJ, 497, 294

Lépine, S., Rich, R. M., \& Shara, M. M. 2003, AJ, 125, 1598

Lépine, S., Rich, R. M., \& Shara, M. M. 2007, ApJ, 669, 1235 
Lépine, S., \& Shara, M. M. 2005, AJ, 129, 1483

Lodders, K. 1999, ApJ, 519, 793

Lodders, K. 2002, ApJ, 577, 974

López-Morales, M. 2007, ApJ, 660, 732

Marcy, G. W., Butler, R. P., Vogt, S. S., Fischer, D., \& Lissauer, J. J. 1998, ApJ, 505, L147

Marcy, G. W., \& Chen, G. H. 1992, ApJ, 390, 550

Martinache, F., Lloyd, J. P., Ireland, M. J., Yamada, R. S., \& Tuthill, P. G. 2007, ApJ, 661, 496

Mayor, M., Marmier, M., Lovis, C., et al. 2011, arXiv:1109.2497

McCarthy, D. W., Jr., Henry, T. J., Fleming, T. A., et al. 1988, ApJ, 333, 943

McLean, I. S., McGovern, M. R., Burgasser, A. J., et al. 2003, ApJ, 596 561

Merrill, K. M., \& Ridgway, S. T. 1979, ARA\&A, 17, 9

Mohanty, S., \& Basri, G. 2003, ApJ, 583, 451

Mould, J. R. 1976a, A\&A, 48, 443

Mould, J. R. 1976b, ApJ, 207, 535

Muirhead, P. S., Edelstein, J., Erskine, D. J., et al. 2011, PASP, 123, 709

Mullan, D. J., \& MacDonald, J. 2001, ApJ, 559, 353

Neves, V., Bonfils, X., Santos, N. C., et al. 2012, A\&A, 538, 25

Nordström, B., Mayor, M., Andersen, J., et al. 2004, A\&A, 418, 989

Nutzman, P., \& Charbonneau, D. 2008, PASP, 120, 317

Ochsenbein, F., Bauer, P., \& Marcout, J. 2000, A\&AS, 143, 23

O’Connell, D. J. K. (ed.) 1958, Proc. Conf. Vatican Obs., Ricerche Astronomich, Vol. 5 (Amsterdam: North Holland)

Önehag, A., Heiter, U., Gustafsson, B., et al. 2011, arXiv:1112.0141

Poveda, A., Herrera, M. A., Allen, C., Cordero, G., \& Lavalley, C. 1994, RevMexAA, 28, 43

Rajpurohit, A. S., Reylé, C., Schultheis, M., \& Allard, F. 2010, in SF2A-2010: Proc. Annual Meeting of the French Society of Astronomy and Astrophysics, ed. S. Boissier et al. (Les Ulis: EDP Science), 275
Reid, I. N., Hawley, S. L., \& Gizis, J. E. 1995, AJ, 110, 1838

Reid, I. N., Hawley, S. L., \& Gizis, J. E. 1997, VizieR Online Data Catalog, 3198, 0

Reiners, A. 2007, A\&A, 467, 259

Ribas, I. 2006, Ap\&SS, 304, 89

Rojas-Ayala, B., Covey, K. R., Muirhead, P. S., \& Lloyd, J. P. 2010, ApJ, 720, L113

Ruiz, M. T., \& Anguita, C. 1993, AJ, 105, 614

Sandage, A., \& Fouts, G. 1987, AJ, 93, 74

Santos, N. C., Israelian, G., \& Mayor, M. 2004, A\&A, 415, 1153

Schlaufman, K. C., \& Laughlin, G. 2010, A\&A, 519, A105

Ségransan, D., Delfosse, X., Forveille, T., et al. 2000, A\&A, 364, 665

Shkolnik, E. L., Hebb, L., Liu, M. C., Reid, I. N., \& Collier Cameron, A 2010, ApJ, 716, 1522

Skrutskie, M. F., Cutri, R. M., Stiening, R., et al. 2006, AJ, 131, 1163

Skumanich, A. 1972, ApJ, 171, 565

Sousa, S. G., Santos, N. C., Mayor, M., et al. 2008, A\&A, 487, 373

Stauffer, J. R., \& Hartmann, L. W. 1986, ApJS, 61, 531

Tsuji, T., Ohnaka, K., \& Aoki, W. 1996, A\&A, 305, L1

Vacca, W. D., Cushing, M. C., \& Rayner, J. T. 2003, PASP, 115, 389

Valenti, J. A., \& Fischer, D. A. 2005, ApJS, 159, 141

van Altena, W. F., Lee, J. T., \& Hoffleit, E. D. 2001, VizieR Online Data Catalog, 1238,0

van Leeuwen, F. 2007, A\&A, 474, 653

Weisberg, S. 2005, Applied Linear Regression, Wiley Series in Probability and Statistics (New York: Wiley-Interscience)

West, A. A., Hawley, S. L., Bochanski, J. J., et al. 2008, AJ, 135, 785

Woolf, V. M., Lépine, S., \& Wallerstein, G. 2009, PASP, 121, 117

Woolf, V. M., \& Wallerstein, G. 2005, MNRAS, 356, 963

Woolf, V. M., \& Wallerstein, G. 2006, PASP, 118, 218

Wright, J. T. 2005, PASP, 117, 657 